Abstracts from the XXI National Congress and VIII
International of the Spanish Society of Conservative
Dentistry, Granada, Spain, May 19-21, 2016 


\section{DIAGNOSIS AND MEDICAL COMPLICATIONS}

\section{- Oral Presentation 1}

TITLE: Association between diabetes and the failure of the root canal treatment: systematic review and meta-analysis

AUTHORS: Saúco Márquez JJ, Cabanillas Balsera D, Martín González J, Jiménez Sánchez MC, Segura Egea JJ.

\section{Objectives}

The aim of this systematic review and meta-analysis is to analyze scientific available evidence on the association between diabetes and the failure of the root canal treatment, evaluating the presence of radiolucent periapical lesions in root-filled teeth.

\section{Materials and Methods}

The review question is: in adult patients who had endodontically treated teeth, does the absence or presence of diabetes, result in an increase in the prevalence of RPL associated to RFT?. A systematic MEDLINE/PubMed, Wiley Online Database, Web of Science and Scopus search is conducted using the following MeSH and key words: Diabetes Mellitus OR Diabetes OR Diabetic OR Hyperglycemia, AND Endodontics, Periapical Periodontitis, Periapical Diseases, Apical Periodontitis, Peri-radicular Lesion, Periapical Radiolucency, Radiolucent Periapical Lesion, Root Canal Treatment, Root Canal Preparation, Root Canal Therapy, Root Filled Teeth, Endodontically Treated Teeth. Seven studies reporting data on the prevalence of radiolucent periapical lesions associated to root-filled teeth both in diabetic and control subjects are included.

\section{Results}

After the study selection, seven epidemiological studies fulfilled the inclusion criteria, representing data from 1593 root canal treatments, 1011 in non-diabetic control subjects and 582 in diabetic patients. The calculated pooled odds ratio $(\mathrm{OR}=1.42 ; 95 \% \mathrm{CL}=1.11-1.80 ; p=$ 0.0058 ) indicates that diabetic patients has significantly higher prevalence of root-filled teeth with radiolucent periapical lesions than controls.

\section{Conclusions}

Available scientific evidence indicates that diabetes is significantly associated to higher prevalence of periapical radiolucencies in endodontically treated teeth, being an important putative pre-operative prognostic factor in root canal treatment. Taking into account that diabetes is the third most prevalent chronic medical condition among dental patients, endodontic providers should be aware of the relationship between the outcome of endodontic treatment and diabetes.

\section{- Oral Presentation 2 \\ TITLE: Evaluation of periapical status of dia- betic versus non-diabetic patients}

\author{
AUTHORS: De la Torre de la Fuente F, Estévez Lua- \\ ña R, Segura Egea J, Cisneros Cabello R.
}

\section{Objectives}

In this study we aim to evaluate the prevalence of apical periodontitis in diabetic patients compared with nondiabetic. We also want to see if the socio-demographic, clinical and therapeutic factors influence the prevalence in both groups. And finally we also analyze the relationship of endodontic treatments present in both groups and their relationship with apical periodontitis.

\section{Materials and Methods}

We took a sample of 47 diabetic type I and II and we matched for age and sex with a sample of 47 non-diabetic patients. With a form analyze the factors relevant to the study such as smoking, obesity, frequency of dental visits etc ... Also a dental valuation which point in a odontogram the presence of caries is performed, periodontal disease and all treatments restorers. Each patient receives a periapical series and endodontic treatments and the presence of apical periodontitis in each tooth are valued. To complete the study diabetic patients are asked to assess the latest relevant analytical para-meters control their disease. We analyze all the data and apply the PAI index to assess the presence of apical periodontitis.

\section{Results}

We get a higher prevalence of apical periodontits in diabetic patients $(p=.004)$. In addition we found that there is an association between apical periodontitis and have a root canal performed $(p=.0001)$ in both groups. Among the factors we studied obtain an association of apical periodontitis with smoking $(p=.007)$ and obesity $(p=.009)$.

\section{Conclusions}

According to this study the diabetic patient, smoker and obese have a higher prevalence of apical periodontitis. As further studies needed to confirm the results.

\section{- Oral Presentation 3}

\section{TITLE: Association between obesity and peria- pical status}

\section{AUTHORS: Sanchez Dominguez B, de la Torre de la Fuente F, Cisneros Cabello R, Martin González J, Segura Egea JJ.}

\section{Objectives}

The aim of this study was to analyze the prevalence of apical periodontitis (PA) in a group of patients and correlate it with the body mass index (BMI). 


\section{Materials and Methods}

The cross-sectional study included 47 patients. The variables weight, height, waist circumference and body mass index were evaluated. According to the BMI, three groups were established: normal weight $(\mathrm{BMI} \leq$ $25, \mathrm{n}=18)$, overweight (BMI $\geq 25, \mathrm{n}=26)$ and obesity $(\mathrm{BMI} \geq 30, \mathrm{n}=3)$. Periapical status was determined by an intraoral radiographic serie (Kodak 6000), assessing the periapex of each tooth, excluding third molars, and using the periapical Index (PAI). Observers were previously calibrated, obtaining an acceptable match (Cohen's Kappa test, $\mathrm{k}=0.77$ ). The results were analyzed statistically using the ANOVA test and logistic regression analysis, considering significant a value of $p \leq 0.05$.

\section{Results}

The prevalence of apical periodontitis (PA) correlated significantly with BMI $(p<0.05)$. Patients in the normal weight group had a prevalence of PA of $16.7 \%$, while in patients with overweight or obesity, the prevalence of PA was $38.5 \%$ and $66.7 \%$, respectively $(p=0.046)$.

\section{Conclusions}

Periapical status correlated significantly with body mass index: the greater body mass index, the higher the prevalence of periapical lesions. Although this study is cross-sectional and cannot lead to conclusions about causality, it is useful to propose hypotheses about causality which should be tested in longitudinal studies.

\section{- Oral Presentation 4}

TITLE: Anatomical variability of lower molars in Spanish population using CBCT

\section{AUTHORS: Clavero González J, Bravo Pérez M, Pérez Heredia M, Ferrer Luque CM, Castelo Baz P, Ruiz Piñón M, Uroz Torres D, Baca García P.}

\section{Objectives}

Identify anatomical variations of first and second mandibular molars in Spanish population by using CBCT.

\section{Materials and Methods}

121 CBCT of mandibular first molars (M1) and 121 of mandibular second molars(M2) of 112 patients with bilateral lower molars without endodontic treatment that CBCT was performed by surgical or orthodontic reasons, were used. Number of roots, number of canals per root and canal configuration was analyzed.

\section{Results}

242 lower molars and 468 roots (245 in M1 and 223 in M2) were analyzed. $94 \%$ of M1 and $83 \%$ of M2 had 2 separate roots. All the mesial roots of M1 presented two ducts and $72 \%$ of distal roots just one. In second molars, $97 \%$ of mesial root showed 2 canals and $92 \%$ of distal roots only one.
Regarding the canal morphology, the mesial roots presented Vertucci configuration type II in $51 \%$ of M1 and $78 \%$ in M2. The distal roots showed Vertucci type I in $72 \%$ of M1 and $92 \%$ of M2.

\section{Conclusions}

CBCT is usefull to detect possible anatomical variations of great diagnostic value for the correct endodontic treatment. Mandibular molars of the Spanish population are similar to those of other populations studied, except morphology of $\mathrm{M}$ roots.

\section{- Oral Presentation 5 \\ TITLE: Morphology of upper molars in Spa- nish population by CBCT}

AUThORS: Pérez Heredia M, Bravo Pérez M, Clavero González J, Castelo Baz P, Baca P, Ruiz Piñón M, Uroz Torres D, Ferrer Luque CM.

\section{Objectives}

Identify anatomical variations of upper molars in the Spanish population by CBCT.

\section{Materials and Methods}

142 CBCT first molars (M1) and 142 second molars (M2) of 112 patients with bilateral upper molars without treatment of root canals that CBCT was performed by surgical or orthodontic reasons, were used. Number of roots, number of ducts and duct system configuration was analyzed.

\section{Results}

284 upper molars and 791 molar roots (419 in M1 and 372 in M2) were analyzed. $97 \%$ of M1 and $79 \%$ of M2 had 3 separate roots. $17 \%$ of M2 a single root. In M1 with 3 separate roots, $100 \%$ of palatal and $97 \%$ of distobuccal roots had a single duct. $86 \%$ of mesiobuccal roots had two canals. M2 with 3 separate roots, all the palatal and distobuccal had one canal. $53 \%$ of mesiobuccal presented a canals, $46 \% 2$ canals and $1 \%$ three canals.

Morphology of the duct system: configuration Vertucci type I in $100 \%$ of P root of M1 and M2, 97\% of distobuccal roots of M1 and $100 \%$ of M2. The mesiobuccal root is the most variable: In M1 $14 \%$ were type I, $57 \%$ type II and 23\% type IV; In M2 53\% type I, 33\% type II and $10 \%$ type IV.

\section{Conclusions}

The upper molars in Spanish population have anatomical variations, mainly in the mesiobuccal root for both $\mathrm{M} 1$ and M2. The CBCT is a complementary diagnostic method very useful to determine the configuration of root canals. 


\section{- Oral Presentation 6}

TITLE: Comparison of different radiographic techniques in the detection of endo-perio lesions

\section{AUTHORS: Santos Pereira J, Antunes Guimarães D, Martins A, Vasconcelos N, Moura Teles A.}

\section{Objectives}

Making a comparison of available radiographic techniques, namely, the analogic radiography (ortopantomography, periapical, occlusal and interproximal radiography) and tri-dimensional digital radiography, in order to realize which one is the most suitable for the detection of endo-perio lesions.

Alert, also, for the inherent risks when using each ones, emphasizing some selection criteria.

\section{Materials and Methods}

The bibliographic research was carried out in the search engines b-On, National Library of Medicine's, PubMed Database and SciELO, with recourse to the following keywords, combined in different ways: "Endodontics", "Periodontics", "Volumetric tomography", "Conebeam", "Conventional x-ray", "Digital x-ray", "Periapical lesions", "Diagnosis", "Radiology" and "Endo-Perio". 48 articles were selected and 3 books were used to help to complete the investigation. The inclusion criteria, with relevant content, that limit the research were: English, Portuguese and Spanish language and the year of publication comprehended between and 2010 and 2015.

\section{Results}

The analogic radiography continues to be, globally, the preferred for the diagnosis of periapical lesions. Between all, the periapical technique has higher sensitivity and specificity having regard to its cost and radiation dose. The tri-dimensional digital radiography must be used as a last resource because, even being highly precise, its cost and radiation dosage are substantially higher.

\section{Conclusions}

Despite the evolution and discovery of new radiological techniques, there's no one that is completely sensible for the diagnosis of endo-perio lesions and so its selection must be weighted and justified.

\section{- Oral Presentation 7}

TITLE: Systemic medication in endodontic emergencies

AUTHORS: Martín González J, Jiménez Sánchez MC, Sánchez Domínguez B, Crespo Gallardo I, Martín Jiménez M, Segura Egea JJ.

\section{Objectives}

he objective of this review is to establish guidelines for prescribing medication in endodontic emergencies.

Materials and Methods

A literature review focused on the use of antibiotics, anti-inflammatories, and analgesics most commonly used in the field of endodontics. The scientific material was obtained from MEDLINE and PubMed data. Twelve items have been analyzed (2002-2016) including systematic reviews or clinical trials and review articles or expert opinions.

\section{Results}

There is no scientific evidence that antibiotics improve pulp pain in patients with irreversible pulpitis. Was not found, in the absence of signs of systemic involvement, significant differences regarding pain or inflammation in patients who were prescribed antibiotics against which no prescribed after root canal treatment. The most effective analgesic for the control of the pulp and / or periodontal pain is the ibuprofen a dose of $600 \mathrm{mg}$ every 8 hours. Centrally acting analgesics only would be indicated in the pre postoperative intense and uncontrollable pain or ibuprofen, exacerbation postoperative pain or acute painful abscesses. The use of preoperative pain medication improves the effectiveness of anesthesia 30 minutes-1 hour before applying.

\section{Conclusions}

In the clinical management of endodontic emergency antibiotics are used excessively. Regarding the use of analgesics and anti-inflammatory, ibuprofen is the drug of choice for pain control pulpal and / or periapical. Also it demonstrated that preoperative analgesic medication improves the effectiveness of anesthesia.

\section{- Oral Presentation 8}

TITLE: Worldwide pattern of antibiotics prescription in endodontic infections

\section{AUTHORS: Crespo Gallardo I, Saúco Márquez JJ, Martín González J, Martín Jiménez M, Segura Egea JJ.}

\section{Objectives}

The aim of this concise review is to analyse the worldwide pattern of antibiotics prescription in endodontic infections.

\section{Materials and Methods}

The PubMed database was searched in January 2016, including the following keywords: [antibiotic OR dentist OR prescribing] AND [endodontic OR pulpitis OR apical periodontitis]. The results were 193 articles using the filter reviews. Over 29 references were retrieved investigating antibiotic prescribing by dentists in endodontic infections. 


\section{Results}

Odontogenic infections and, especially, endodontic infections, are polymicrobial involving a combination of Gram-positive, Gram-negative, facultative anaerobes and strict anaerobic bacteria. Therefore, antibiotics can be used as an adjunct to endodontic treatment. However, most of chronic or even acute endodontic infections can be successfully managed by pulp extirpation, which eliminate the source of infection, followed by drainage of abscess, or tooth extraction, without the need for antibiotics. Literature provides evidence of inadequate prescribing practices by dentists.

Amoxicillin is the drug of choice for endodontic infections in most of the countries, and clindamycin and erythromycin are the choice in patients allergic to penicillin. Dentists worldwide are over-prescribing antibiotics in the management of endodontic infections, although there are no data about dentist's antibiotic prescription patterns in many countries (China, Indonesia, Bangladesh, Russia, Japan, Mexico).

\section{Conclusions}

It is necessary an improvement of antibiotics prescribing habits in the treatment of endodontic infections, as well as educational initiatives to encourage the coherent and proper use of antibiotics in these conditions.

\section{- Oral Presentation 9}

\section{TITLE: Assessment of success and survival of restorative and endodontic treatments}

\section{AUTHORS: Valero Remohí L, Alegre Domingo T, Faus Matoses V, Faus Matoses N, Faus Llácer VJ.}

\section{Objectives}

The objective of this study is to examine the success and survival of restorative and root canal treatments with a 3 year follow up.

\section{Materials and Methods}

Ninety-five medical records of patients treated from 2009 to 2012 fulfilled the inclusion and exclusion criteria. Patients were clinical and radiographically examined after 1 month, 3 months, 6 months, 1 year and 3 years.

During the evaluation, periapical status was controlled. Previous radiographs were compared in order to determine periapical healing. Pain, movility, bleeding and probing and percussion was also examined. Depending on the periapical healing, treatment success or failure was determined.

\section{Results}

Sixty seven teeth with root canal treatment were included in this study. $88.1 \%$ of the teeth with root canal and resto-rative treatment succeeded after a follow-up of 36 months. There was a $90.4 \%$ of success rate in endo- dontic treated teeth and a restorative success of $94.0 \%$. Among patients with a successful endodontic treatment, $98.7 \%$ also achieved a restorative success. On the other hand, among patients with a failed endodontic treatment, only $50 \%$ of them survived. Only complications during endodontic treatment, number of canals of the treated tooth and the use of a post are significantly associated to the probability of success ( $p=0,006, p=0,018$ y $p=0,029$ respectively).

\section{Conclusions}

Root canal treatment is a conservative and predictable procedure with a high success rate. Apical extension of root canal obturations are predictive in endodontic success and periapical healing. 


\section{ELECTRONIC ÁPEX LOCATORS}

\section{- Oral Presentation 10}

TITLE: influence of the diameter of the file on the accuracy of the Raypex $6{ }^{\circledR}$

\author{
AUTHORS: Martín JA, Benavides-Reyes C, Lucena \\ C, Vidal C, González-Rodríguez MP.
}

\section{Objectives}

The aim of this study was to evaluate the influence of the diameter of the file on the accuracy of the Raypex $6 \circledR$ electronic apex locator in canals with large foramen and without tapering.

\section{Materials and Methods}

Thirty bovine mandibular incisors were used. With a dental bur, a coronal reference plane in the enamel-cementum junction were obtained and $5 \mathrm{~mm}$ were removed to achieve canals without tapering and large foramen. With magnification (20x), the file that adjusted to the apical diameter was selected, and the actual lenght was made.

By other operator, the molars were embedded in fresh alginate and the electronic measurement was made using the 'constriction' mark of the Raypex6 as reference. First, it was measured with a K\#10 file and then, with the K-file that fitted to the apical diameter in each case. The difference between the electronic measurements with both files and the actual length were calculated. The influence of the apical gauge was analized with one-way-ANOVA test. The Shapiro-Wilk test was used to check the normality of the quantitative data distribution. Means comparison was analized using the t-test for paired samples. The significance level was set at $p<0.05$.

\section{Results}

The mean of the apical gauge was $0.77 \mathrm{~mm}$. Apical size did not interaction in the measurement with $\mathrm{K} \# 10$ file $(p=0.177)$, although it interactioned with the snugfitting file $(p<0.001)$.

The mean difference between the K\#10-file and the actual length was $-0.53 \mathrm{~mm}$, and between the snug-fitting file and the actual length was $-0.37 \mathrm{~mm}$, but no significant differences were found $(p=0,271)$.

\section{Conclusions}

In these experimental conditions, the use of a snug-fitting file did not increase the accuracy of the measurement respect the use of a K\#10 file.

\section{- Oral Presentation 11 \\ TITLE: Accuracy of the Root ZX apex locator on widened foramina in the presence of blood}

\author{
AUTHORS: Herrera Martínez M, Bonilla Represa \\ V, Jiménez Planas A, Ábalos Labruzzi C.
}

\section{Objectives}

To evaluate the accuracy of the Root ZX apex locator (J. Morita Corp, Tokyo, Japan) on widened foramina in the presence of blood, taking into consideration the absence of formal related studies in the literature.

\section{Materials and Methods}

Twenty single-root teeth, divided in two groups, were embedded in an alginate mould. The foramina were widened from $0.1 \mathrm{~mm}$ to $1.0 \mathrm{~mm}$. The measurements were taken with all possible file sizes $\geq \# 10(\mathrm{n}=1200)$, in a controlled environment with $5.25 \%$ sodium hypochlorite and another sample blood contaminated. The statistical accuracy of the Root ZX was calculated by the different diameters and by the influence of the file size.

\section{Results}

The accuracy of the Root ZX apex locator on the controlled group with a $\pm 0.5 \mathrm{~mm}$ tolerance was $87 \%$ using a $\leq 0.6 \mathrm{~mm}$ apical foramen and $84 \%$ using $0.7 \mathrm{~mm}$ apical foramen and size 45 files or larger. On the blood contaminated sample, accuracy was not certain and not affected by the apical foreman size. With a $\pm 1 \mathrm{~mm}$ tolerance, the accuracy on a controlled environment with sodium hypochlorite was $99 \%$ using a $\leq 0.6 \mathrm{~mm}$ apical foramen, $98 \%$ using $0.7 \mathrm{~mm}$ apical foramen and size 45 files or larger, and $95 \%$ using $0.8 \mathrm{~mm}$ apical foramen and size 70 files or larger. In the rest, accuracy was not certain. On the blood contaminated sample with a \pm 1 $\mathrm{mm}$ tolerance, was $83 \%$ using a $\leq 0.55 \mathrm{~mm}$ apical foramen. In the rest, accuracy was not certain.

\section{Conclusions}

The Root ZX apex locator was accurate for an apical size of $\leq 0.6 \mathrm{~mm}$, independently of the file size; between 0.7 to $0.8 \mathrm{~mm}$, we should adjust the files to the foramen, whereas above size $0.9 \mathrm{~mm}$ the locator is not accurate in a controlled environment sodium hypochlorite and on a blood contaminated sample the accuracy is considerably affected.

\section{- Oral Presentation 12}

TITLE: Cyclic fatigue resistance of rotary systems with different processing Nickel-titanium alloy

AUTHORS: Ruiz Sánchez C, Faus Matoses V, Alegre Domingo T, Faus Matoses VJ. 


\section{Objectives}

The purpose of this study was to compare the cyclic fatigue resistance of 3 nickel-titanium endodontic instrument, ProTaper Next (Dentsply Maillefer, Ballaigues, Switzerland), Profile Vortex Blue (DentsplyTulsa Dental, Tulsa, OK, USA) and ProTaper Universal (Dentsply Maillefer, Ballaigues, Switzerland).

\section{Materials and Methods}

Cyclic fatigue test was conducted by operating instruments from ProTaper Next X2, Profile Vortex Blue 25.06 and ProTaper Universal F2. A total of $234(n=78)$ instruments were rotated in 2 simulated stainless steel curved canals with different angles of curvature $\left(45^{\circ}\right.$ and $\left.60^{\circ}\right)$ and $5-\mathrm{mm}$ radius of curvature. The number of cycles to fracture (NCF) was calculated. Data were compared by using 2-way analysis of variance and Bonferroni test. Statistical significance was set at $p<0.05$.

\section{Results}

Profile Vortex Blue showed better resistance to cyclic fatigue in both curved canals than ProTaper Next and ProTaper Universal. The lowest resistance to cyclic fatigue canals were obtained by ProTaper Universal.

\section{Conclusions}

From the tested rotary files, Profile Vortex Blue was the most resistant to fatigue failure; and ProTaper Next showed higher number of cycles to failure than ProTaper Universal.

\section{- Oral Presentation 13}

TITLE: Alternating versus continuous rotation: centering ratio and canal transportation with the ProTaper Next

\section{AUTHORS: González Chapela J, Castelo Baz P, Va- rela Patiño P, Martín Biedma B, Ruíz Piñón M.}

\section{Objectives}

The technique of alternating rotation has been used with NiTi rotary instruments to increase resistance to fracture compared with conventional continuous rotation. The aim of this study was to determine the influence of the type of rotation on canal transportation and centering ability using cone-beam computed tomography (CBCT) imaging.

\section{Materials and Methods}

In total, 50 mesial canals of mandibular molars with curvatures between $30^{\circ}$ and $60^{\circ}$ were divided into two experimental groups $(n=25)$ according to the type of movement employed with the ProTaper Next instruments: Group A (alternating rotation) and Group B (continuous rotation). Canals were scanned before and after instrumentation using a CBCT scanner to evaluate root canal transportation and the centering ratio at 3,5, and $7 \mathrm{~mm}$ from the apex. Data were analyzed statistically; the significance level was set at $p<0,05$.

\section{Results}

There was no significant difference between the groups in canal transportation or the centering ratio at any of the three studied cross sections. Canal transportation: $3 \mathrm{~mm}, \mathrm{~A}=0.05 \pm 0.04 / \mathrm{B}=0.05 \pm 0.04(p=0.95) ; \mathrm{mm}$, $\mathrm{A}=0.07 \pm 0.04 / \mathrm{B}=0.09 \pm 0.06(p=0.22) ; 7 \mathrm{~mm}, \mathrm{~A}=0.10$ $\pm 0.07 / \mathrm{B}=0.09 \pm 0.07(p=0.48)$. Centering ratio: $3 \mathrm{~mm}$, $\mathrm{A}=0.54 \pm 0.25 / \mathrm{B}=0.58 \pm 0.23(p=0.59) ; 5 \mathrm{~mm}, \mathrm{~A}=0.54$ $\pm 0.23 / \mathrm{B}=0.43 \pm 0.29(p=0.19) ; 7 \mathrm{~mm}, \mathrm{~A}=0.49 \pm 0.21 /$ $\mathrm{B}=0.58 \pm 0.30(p=0.29)$.

\section{Conclusions}

ProTaper Next instruments promoted minimal apical transportation and remained relatively centered within the root canal, with no significant difference between alternating and continuous rotation.

\section{- Oral Presentation 14}

TITLE: Comparison of resistance to cyclic fatigue between two rotary nickel titanium systems

\author{
AUTHORS: Gil Flor J, Martinez Viñarta M, Faus \\ Matoses V, Faus Llácer V.
}

\section{Objectives}

The aim of this study was to compare in-vitro resistance to cyclic fatigue between two systems of nickel-titanium (NiTi) rotary files to create glidepath: Proglider (PG) (Dentsply-Maillefer, Ballaigues, Switzerland) and Pathfile (PF) (Dentsply-Maillefer, Ballaigues, Switzerland) in D4 and D8.

\section{Materials and Methods}

94 instruments PG and PF with tip diameter and taper of 16/02 were used. They were rotated in a stainless steel simulated canal $\left(80^{\circ}\right.$ angle and radius of curvature of $2 \mathrm{~mm}$ ) to $300 \mathrm{rmp}$ and torque of $2 \mathrm{~N} . \mathrm{cm}$ using a X-Smart motor (Dentsply-Maillefer, Ballaigues, Switzerland) with handpiece 16: 1. They were distributed into 2 groups. In the first $(n=54)$ the point of maximum curvature of the instument was set at D8. And in the second $(n=40)$, was fixed in D4. The process was timed until observe the fracture of the instrument and it was resulted in number of cycles to failure. Data were analyzed using SPSS 22 software (Chicago, IL). The significance level was set at $\mathrm{p}$ value $<0.05$ and groups were compared with a 2-way ANOVA test, and Bonferroni test was applied too.

\section{Results}

PG was the most resistant to cyclic fatigue showing a greater number of cycles to failure than PF $(P<0.05)$ in D4. However, PF was stronger than PG $(P<0.05)$ in D8.

\section{Conclusions}

The cyclic fatigue resistance is influenced by the type of instrument, with different alloy, such as the dis- 
tance from the tip to the point of fracture, because the cross section. In conclusion PG (M-Wire NiTi) showed increased resistance to cyclic fatigue that PF (conventional NiTi), where their cross-sections are similar, but different alloys. However, in D8 and due to the smaller cross section, PF is stronger than PG despite being of conventional NiTi.

\section{- Oral Presentation 15}

TITLE: Cyclic fatigue resistance of rotary endodontic instruments with different alloy

\section{AUTHORS: Romero Mora D, Faus Matoses V, Faus Matoses I, Martínez Viñarta M, Faus Matoses VJ.}

\section{Objectives}

The purpose of the study was to compare the cyclic fatigue resistance of Níquel Titanium rotary endodontic instruments with different alloy and design.

\section{Materials and Methods}

The cyclic fatigue tests were carried out with following rotary instruments: Protaper Universal 25.08, Hyflex 25.08, K3XF 25.08 and Helix 25.08. These instruments were chosen for having $0,25 \mathrm{~mm}$ tip diameter and 08 of taper. A total of 140 instruments (n:35), were rotated in a artificial stainless steel curve canal (60o curvature and $5 \mathrm{~mm}$ radius), with $20 \mathrm{~mm}$ in length with a tip of $0.40 \mathrm{~mm}$ and 09 taper, the diameter of the simulated canal was wider than the files allowing free rotation. The canal was stuck in an acrylic surface, where the hand piece was also fixed. The files were tested in the motor (X-smart; Dentsply Maillefer ), using a continuous rotation movement and maximum torque $(5.2 \mathrm{Ncm})$, in a speed of manufactures configuration setup. The time was timing to the hundredth of a second, and was stopped as the fracture was detected. The time was then converted into number of cycles to failure (NCF). Data were analyzed by using 2-way analysis of variance (ANOVA), in software (SPSS 15.0, Chicago, IL) to determinate the number of rotations and time until fracture. Post hoc Tamhane test was applied to identify the groups were more significant. Statistical significance was set at $P$ value less than $5 \%$.

\section{Results}

Statistical analysis of data showed significantly more resistant $(p<0,001)$ to cyclic fatigue from Hyflex files in comparison with the other files tested in the study.

\section{Conclusions}

The study demonstrate that CM-wire manufacturing process files were more resistant to cyclic fatigue than instruments produced by M-Wire alloy and conventional NiTi.

\section{- Oral Presentation 16 \\ TITLE: Apical extrusion of debris caused by several shaping techniques}

\author{
AUTHORS: Moura Teles A, Marques A, Cardoso \\ Silva C, Sequeira T, Silveira A.
}

\section{Objectives}

The debris produced during an endodontic treatment may be, unintentionally, extruded through apical foramen and may function as an irritant factor causing flare-ups or, even worse, interrupt the healing process of a periapical lesion. Quantify and compare the debris extruded through the apical foramen of different techniques: a manual -Roane; a continuous rotary: Protaper ${ }^{\circledR}$ System and two reciprocating: WaveOneTM System and Reciproc $\AA$.

\section{Materials and Methods}

134 incisives were prepared (scaling, incisal edge planing, access cavity, - varnishing, with Wynie $\AA$, the outer region of the apical third with a $\mathrm{K}$ file 015 inserted into the root canal) and shaped by each technique in study, resulting in 4 test groups $(n=31)$ and 2 control ones $(n=5)$. For irrigation, a solution of $3 \%$ sodium hypocloride was used. Debris extruded through apical foramen were collected in Eppendorf tubes.

These, were then, placed in a greenhouse for five days at a constant temperature of $68^{\circ} \mathrm{C}$, and then weighed using an analytical balance digital of high precision. The weight of the debris extruded, were calculated by the difference between the final and initial values. The data were processed using SPSS (C) vs 20.0, using techniques suitable for description and deduction $(\alpha=0.05)$.

\section{Results}

There was debris extrusion through apical foramen in all instrumentation techniques used, but it was observed that the Roane Technique promoted the greater amount of debris, having only made a significant difference $(p<0.05)$ when compared to WaveOneTM and Reciproc $\AA$ Systems. Between them, no statistically significant differences $(p>0.05)$ occured. Statistically significant difference was yes found between the Protaper ${ }^{\circledR}$ and the WaveOneTM.

\section{Conclusions}

Use of a shapping technique that minimizes the apical extrusion of debris will be advantageous for the dentist and to the patient either, as a better post-operative is expected and, hence, a more effective periapical tissues' repairing. 


\section{- Oral Presentation 17}

TITLE: Analysis of dentinal microcrack formation after rotary instrumentation: invasive method versus Micro-CT

\section{AUTHORS: Miguéns Vila R, Varela Patiño P, Caste- lo Baz P, Ruíz Piñón M, Martín Biedma B.}

\section{Objectives}

The aim of this study is to evaluate dentinal microcrack formation after rotary instrumentation with ProTaper Next, using Micro-CT or observation with stereomicroscope after seccional cut of the samples as the two analysis methods.

\section{Materials and Methods}

20 roots were selected, extracted for periodontal reasons, with curvatures greater than $30^{\circ}$. All samples were decoronated and randomly divided into two groups $(\mathrm{n}=10)$. In the Micro-CT (Skyscan 1172) study group, a previous scan of the samples was conducted. All canals were preflared with a \#10 K-File following by a Proglider to create an appropiate glidepath, and instrumented with the ProTaper NEXT system to a final file X2. In the Micro-CT group a postinstrumentation scan was done and compared with the preoperative scan. The other samples were transversely seccioned with a low-speed diamond saw (Isomet 4000) in 3 levels, before subsequent analysis of the samples with a stereomicroscope (Leica MZ16F).

\section{Results}

In the preliminary Micro-CT scans, some microcracks were observed $(9.5 \%)$ in the external walls (probably due to teeth extraction procedures). In the postinstrumentation scan, no new microcracks were found. In the seccional cut group, there were significantly more microdefects $(33.5 \%)$ than in the Micro-CT group $(P<0.05)$.

\section{Conclusions}

Under the limitations of this study, it can be concluded that the ProTaper NEXT rotary system did not generate new dentinal microcracks after Micro-CT analysis.

The use of an invasive method can produce false positives due to the aggressive nature of the cut over dentin and because preexistent defects and the ones produced by instrumentation can not be differenced, opposed to using non-invasive methods.

\section{- Oral Presentation 18}

TITLE: Reciproc ${ }^{\circledR}$ system as a method to remove gutta-percha in endodontic retreatments

\author{
AUTHORS: Alfaro Perea J, Viana Gonçalves Aires \\ AM.
}

\section{Objectives}

This review summarizes the efficacy, remaining volume of filling material and time to perform the procedure of Reciproc ${ }^{\circledR}$ system in removing Gutta-percha from the root canal in endodontic retreatments.

\section{Materials and Methods}

A Pubmed electronic search was conducted by using appropriate key words to identify the available investigations on Reciproc ${ }^{\circledR}$ system in endodontic retreatments. After retrieving the full-text relevating articles, the cross citations were also identified.

\section{Results}

A total of 20 articles published between October 2013 and March 2016 were identified. After full text evaluation, 7 of these were eliminated. The selection process resulted in the final sample of 13 studies.

\section{Conclusions}

Based on the results of this systematic review, none of the retreatment procedures provides complete removal of the filling material.

The Reciproc ${ }^{\circledR}$ and Wave One ${ }^{\circledR}$ reciprocating systems are as affective as the Protaper Universal ${ }^{\circledR}$ retreatment system for gutta-percha and sealer removal.

The Reciproc ${ }^{\circledR}$ system is the most rapid method for removing gutta-percha, and is associated with less debris extrusion when compared with a conventional rotary retreatment system.

\section{- Oral Presentation 19}

TITLE: Instrument Separation during the Non Surgical Endodontic Treatment. Causes, prevention and clinical approach

AUTHORS: Leite A, Martins L, Ribeiro P, Silva B, Teles A.

\section{Objectives}

Description of processes that lead to fracture of instruments, as well as the techniques and tools used in its resolution.

\section{Materials and Methods}

We used search engines like Pubmed and Scielo, with the key words: "broken files", "endodontic file removal", "instrument bypass" in Portuguese, English and Spanish, dated between 2000-2015.

\section{Results}

Intracanalar instrument fracture during the instrumentation phase and chemical-mechanical preparation of Non surgical endodontic treatment presents itself as a common event. The presence of the fragment inside the canal system may interfere with our ability to desinfect the space, conditioning the prognosis of the endodontic treatment. 


\section{ENDODONTIC IRRIGATION}

the main causes of instrument separation are the occurrence of cyclic metal fatigue and tortion phenomenon.

Except when the fragment is in a coronal position or in cases where a straight access is possible, the bypass technique is the first treatment option. If bypass isn't possible, the removal should be attempted. In extreme cases and according to specific parameters, the maintenance of the fragment "in situ" may be reserved only as a last resource.

\section{Conclusions}

Once the ultimate goal of endodontic treatment is canalar desinfection, factors such as anatomy, fragment location or treatment stage in which separation occurred, should be taken into account when deciding the most appropriate procedure for the case.

\section{Objectives} not implemented the apical third.

\section{- Oral Presentation 20 \\ TITLE: Effectiveness of surfactants in irrigant penetration}

\author{
AUTHORS: Estevez R, Conde A, Escorial A, De la \\ Torre F, Cisneros R.
}

Analyze the ability of different irrigation techniques and solutions to dissolve organic matter located in areas

Measure whether the use of surfactants favors the penetration of irrigant inside the ductwork.

Evaluate the time necessary to remove organic tissue in areas not instrumented by passive ultrasonic activation.

Evaluate the clinical application that can be obtained from this study establishing effective to obtain a proper cleaning of the last apical millimeter guidelines.

\section{Materials and Methods}

30 samples divided into 4 groups: control $(n=10)$, positive pressure irrigation $(n=40)$, passive ultrasonic activation for 15 seconds $(n=40)$ and passive ultrasonic activation for 30 seconds $(n=40)$. The control group only was irrigated with distilled water. The other three groups were divided into two groups: group A (final irrigation with $5,85 \%$ sodium hypochlorite) and group B (final irrigation with Chlor-Xtra $\left.{ }^{\circledR}\right)$. Weight evolution was studied by analysis of variance (ANOVA).

\section{Results}

All groups where surfactants are used as irrigating show higher dissolution. Statistically significant differences between activation groups and positive pressure irrigation $(p<0.001)$.

\section{Conclusions}

11 technical and irrigation solutions employed in this study are capable of dissolving the organic tissue located in areas not instrumented the apical third. There is increased dissolving pulp tissue in those groups wherein the surfactant agent in combination with sodium hypochlorite is used. Statistically significant differences in favor of passive ultrasonic activation surfactant for 15 seconds compared to the activation of hypochlorite for 30 seconds. A passive ultrasonic activation sodium hypochlorite combined with surfactant for 15 seconds, seems an effective technique to remove large amount of organic tissue located in areas not instrumented simulated. 


\section{- Oral Presentation 21}

TITLE: Molecular detection of microbiota in teeth treated by photodynamic therapy

AUTHORS: Zorita García M, Campo Moreno R, Cobo Álvarez M, Alonso Ezpeleta LO, Rico Romano C, Mena Álvarez J, Zubizarreta Macho A.

\section{Objectives}

Identify the bacteria present in the radicular canals of teeth with chronic apical periodontitis, treated with photodynamic therapy as an adjunct of the endodontic therapy.

Materials and Methods

Forty-two patients were treated with photodynamic therapy as an adjunct of endodontic treatment, in order to improve the microbiological eradication. Three different microbiological samples were collected from each patient from the radicular canals in different stages: 1) at the camera opening, 2) after the irrigation protocol, and 3) after the photodynamic therapy. All samples were treated with molecular techniques of polymerase chain reaction (PCR), denaturing gradient gel electrophoresis (DGGE) and nucleotide sequence to identify the bacteria present in the radicular canals.

Total DNA was obtained using the QIAamp kit (Qiagen). The whole bacterial population was determined by PCR-DGGE technique with universal 16S-rRNA Gene Primers. Amplicons were separated in $8 \%$ vertical electrophoresis polyacrylamide gels at $60^{\circ} \mathrm{C}$; with a ureaformamide denaturing gel gradient of $30-50 \%$ at $130 \mathrm{~V}$ during 16h. Bands were excised, re-amplified with the same primers, sequenced and results were compared with those from the GenBank NCBI database to assign the corresponding genera and species. Presence of Enterococcus faecalis and Enterococcus faecium was also tested by specific PCR reactions.

\section{Results}

Bacterial species detected were as follows: Staphylococcus epidermidis, Staphylococcus warneri, and Staphylococcus lugdunensis. Ambiental bacteria as Bacillus thuringiensis, Brevibacillus brevis and Escherichia fergusonii. Presence of E. faecalis or E. faecium was not detected by DGGE and this result was confirmed by negative specific PCR amplification.

\section{Conclusions}

Enterococcus are not present in the first stage of the first endodontic infection, being the Staphylococcus genera the most frequently detected in teeth with chronic apical periodontitis, treated with photodynamic therapy as an adjunct of the endodontic therapy.

\section{- Oral Presentation 22}

TITLE: Efficacy of different irrigants in the removal of calcium from two calcium hydroxide intracanal dressing

\author{
AUTHORS: Benavides-Reyes C, Sanchez-Sanchez \\ P, González-Rodríguez MP, Bolaños-Carmona V, \\ González-López S.
}

\section{Objectives}

The aim of this study was to evaluate the efficiencies of different irrigation solutions for the removal of calcium of two intracanal dressings based on calcium hydroxi-de.

\section{Materials and Methods}

Samples of Calcicur and UltraCal XS were dried in an oven at $40^{\circ} \mathrm{C}$ for 3 days and ground. Samples of 8.00 $\pm 0.20 \mathrm{mg}$ of both products $(\mathrm{n}=15)$ were obtained. The samples were randomly divided into 5 groups $(n=3)$ according to the irrigation solution: $2.5 \% \mathrm{NaOCl}, 5 \%$ $\mathrm{NaOCl}, 17 \%$ EDTA, 7\% maleic acid and distilled water.

Calcicur and Ultracal XS samples were mixed with 1 $\mathrm{mL}$ of each irrigant for 1 minute at $37^{\circ} \mathrm{C}$. The calcium ion release was measured by means of inductively coupled plasma optical emission spectrometry (ICP-OES), and $\mathrm{pH}$ values were also measured.

Respective efficiencies on calcium ion release were evaluated using One Way ANOVA and Bonferroni tests. Intergroup comparisons were performed with the Student t-test.

\section{Results}

For both products, solutions of $7 \%$ maleic acid and $17 \%$ EDTA were superior to $2.5 \% \mathrm{NaOCl}, 5 \% \mathrm{NaOCl}$ and distilled water in removing calcium hydroxide $(p<0.001)$. There were no significant differences among the other groups ( $>0.05)$.

\section{Conclusions}

Irrigation solutions of 7\% maleic acid and $17 \%$ EDTA can prove more effective in the removal of calcium than $2.5 \% \mathrm{NaOCl}, 5 \% \mathrm{NaOCl}$ or distilled water.

\section{- Oral Presentation 23}

TITLE: Root canal disinfection with sodium hypochlorite and maleic acid solutions

AUTHORS: Solana CM, González-Castillo S, Ternero Escudero FJ, Ferrer-Luque CM, Ruíz-Linares M, Arias Moliz MT, Baca P.

\section{Objectives}

To assess the antimicrobial residual effects of maleic acid solutions in alternated and conventional irrigation protocols with $2.5 \%$ sodium hypochlorite. 


\section{Materials and Methods}

Fifty-six single-rooted teeth were cut at the enamelcementum junction to obtain roots of $12 \mathrm{~mm}$ in length. The specimens were infected during four weeks with Enterococcus faecalis to evaluate antimicrobial effects and then were randomly assigned to 4 four study groups $(n=12)$ and one control group $(n=8)$. The root canals were instrumented with the Protaper system. The irrigation protocols were: Group 1, 2.5\% $\mathrm{NaOCl}$ during instrumentation and $7 \%$ maleic acid at the end of the preparation; Group 2, 2.5\% NaOCl followed by maleic acid to $7 \%$ during instrumentation and final irrigation with maleic acid + cetrimide + chlorhexidine; Group 3 $2.5 \% \mathrm{NaOCl}$ followed by $7 \%$ maleic acid $+0.2 \%$ cetrimide during instrumentation and final irrigation with maleic acid + cetrimide + chlorhexidine; Group 4, saline solution during and after instrumentation. The irrigation effectiveness was determined immediately after preparation and during 80 days. The proportion of samples without bacterial regrowth was evaluated using survival analysis Kaplan-Meier. Differences between groups were analyzed by the log-rank test.

\section{Results}

After instrumentation only the saline solution group exhibited growth of bacteria in all samples, whereas it was not isolated in any other groups. All the negative controls exhibited no bacterial growth throughout the study. Over time, the group 1 showed $50 \%$ of samples regrown by day 18 , while the two alternated irrigation protocols obtained a low number of regrown samples at 80 days, with no significant differences between them.

\section{Conclusions}

Maleic acid solutions in alternating irrigation regimens with $2.5 \%$ sodium hypochlorite using final irrigation with maleic acid + chlorhexidine+ cetrimide are effective protocols to long-term control of infection in root canals.

\section{- Oral Presentation 24}

TITLE: Effectiveness of irrigation regimens in root canal preparation

AUTHORS: Ternero Escudero FJ, Solana CM, Pérez-Heredia M, Baca P, Ruíz-Linares M, FerrerLuque CM.

\section{Objectives}

To evaluate the ability to remove the smear layer of maleic acid solutions and sodium hypochlorite in alternated and conventional irrigation regimens in root canal preparation.

\section{Materials and Methods}

Forty single-rooted teeth were cut at the enamel-cementum junction to obtain roots of $12 \mathrm{~mm}$ in length.
The specimens were randomly assigned to 4 four study groups $(n=10)$. The root canals were instrumented up to F3 file using the ProTapersystem.The irrigation protocols were: Group 1, 2.5\% NaOCl during instrumentation and $7 \%$ maleic acid as final irrigating solution; Group 2, $2.5 \% \mathrm{NaOCl}$ followed by $7 \%$ maleic acid during instrumentation and final irrigation with maleic acid + cetrimide + chlorhexidine; Group 3, 2.5\% NaOCl followed by $7 \%$ maleic acid $+0.2 \%$ cetrimide during instrumentation and final irrigation with maleic acid + cetrimide+ chlorhexidine; Group 4, saline during and at the end of the preparation. All specimens were sectioned into two halves to be observed by scanning electron microscope. The cleaning was evaluated in the coronal middle and apical thirds of the root canals. Photomicrographs were obtained at 2000 magnification and were evaluated following a previously established scale. Data were analyzed by the U de Mann-Whitneyand Friedman tests.

\section{Results}

The saline solution group (control) showed not ability to remove the smear layer. There were no statistically significant differences between groups for coronal $(p=0,395)$, middle $(p=0,191)$ and apical $(p=0,806)$ thirds in order to remove the smear layer. In all groups the best results were obtained at the coronal third, followed by the middle and apical third.

\section{Conclusions}

Maleic acid solutions in alternating with 2,5\% $\mathrm{NaOCl}$, using a final solution of maleic acid + cetrimide + chlorhexidine, provides effective protocols to remove the smear layer of instrumented root canals.

\section{- Oral Presentation 25}

TITLE: Effectiveness of phytic and etidronic acids against polymicrobial biofilms

\section{AUTHORS: Valriberas Herrero C, García Vázquez M, Ruiz-Linares M, Baca P, Aguado B, Ferrer Luque CM.}

\section{Objectives}

The aim of this study is to evaluate and compare the antimicrobial effect of phytic and etidronic acids, alone and followed by $2 \%$ chlorhexidine on polymicrobial biofilms formed in dentin.

\section{Materials and Methods}

Polymicrobial biofilms, from bacterial samples collected from infected root canals, were grown on standardized radicular dentin blocks for 3-weeks. Antibiofilm activity of $18 \%$ etidronic acid and $1 \%$ phytic acid, alone and followed by $2 \%$ chlorhexidine, was determined by Adenosine triphosphate assay (ATP) and anaerobic culture counts (CFUs). Two specimens were analyzed under confocal scanning laser microscopy (CSLM). The 
results of bioluminescence and CFUs were analyzed using the ANOVA test and in order to compare the efficacy of the different protocols the Mann-Whitney test was used. The correlation between the two methods was determined by the Pearson's correlation coefficient.

\section{Results}

A high Pearson's correlation level was obtained between the results of bioluminescence and CFUs counts $(\mathrm{r} 2=0,812, p<0.001)$. One percent phytic acid followed by $2 \%$ chlorhexidine, showed the best results in reducing metabolic activity by ATP assay. The exposure to $2 \%$ chlorhexidine significantly reduced CFUs counts obtained with phytic and etidronic acids. The images obtained with CSLM were consistent with these results.

\section{Conclusions}

The present study shows that, $2 \%$ chlorhexidine improved antimicrobial activity of phytic and etidronic acids against a polymicrobial root canal biofilm.

\section{ENDODONTIC OBTUR ATION-OTHERS}

\section{- Oral Presentation 26 \\ TITLE: Centering of the carrier in curved ca- nals: Thermafil vs GuttaCore}

\author{
AUTHORS: Dablanca Blanco AB, Castelo Baz P, \\ Martin Biedma B, Rivas Mundiña B, Varela Patiño \\ P.
}

\section{Objectives}

Comparison of the behavior of two endodontic obturation systems (Thermafil VS GuttaCore) in cases of curved canals. The aim is observing the relationship between the components of these obturators (carrier and guttapercha) and its adaptation to the canal walls. The degree of centering carrier inside the canal is quantified, and it will be related to the filling material and the degree of root curvature.

Materials and Methods

The sample consists of a total of 40 single-rooted teeth with a root curvature comprised between 20 and 45 degrees. The entire sample is instrumented until F4 of Protaper Universal and it is sealed with obturation systems to compare (20 teeth with Thermafil system and 20 teet with GuttaCore). Each root is cross sectioned $1 \mathrm{~mm}, 4 \mathrm{~mm}$, and $7 \mathrm{~mm}$ from apex in order to observe the behavior of obturator along the canal, and phtographed with a $25 \mathrm{x}$ magnification. The distance between the center of the canal and the center of carrier is measured, obtaining a measure in milimeters that will indicate the offset from the core of these materials. Data are studied using ANOVA and T-Student Test statistical analyses.

\section{Results}

There are no significant differences $(p>0.05)$ between two materials with regarding to off.-centering carrier $(0.0009+-0.0113)$. The off-centering is significantly higher $(p<0.05)$ in curvatures of more than $29^{\circ}$ $(0.106 \mathrm{~mm})$ comparing to the ones that are less than $29^{\circ}$ (0.076mm).

\section{Conclusions}

No significant differences in the behavior of both materials in curved canals. The carrier obturation systems'offcentering degree of the core is greater as the degree of the root curvature to be sealed increases.

\section{- Oral Presentation 27 \\ TITLE: Pulp Capping Materials}

AUTHORS: Aires A, Alfaro J, Ramírez Sebastia A, Côrte Real L, Durán Sindreu F. 


\section{Objectives}

The objective of this paper is to present and compare the new materials used in direct pulp capping.

\section{Materials and Methods}

Literature review in PubMed using the keywords: "pulp capping", "calcium hydroxide", "lasers", "biodentine", "MTA", "Theracal", "calcium phosphate". The number of articles reviewed was 15 .

\section{Results}

According to the ESE (European Society of Endodontology), direct pulp capping is a clinical procedure in which the pulp is covered with a protective material directly into the exposure area. Healing does not directly depends on the effect of the material but on its ability to prevent bacterial infiltration. So the goal is to maintain healthy tooth pulp (the pulp leaving their recognized active regeneration capacity) after this have suffered exposure (trauma, decay, accidental exposure during tooth preparation).

The selection of material is considered very important to minimize and eliminate the pulp inflammation factor. Materials containing calcium hydroxide $(\mathrm{Ca}(\mathrm{OH})$ 2) gained importance after the investigation of Hermann et al in 1930. Since then calcium hydroxide remained the "gold standard". In the most current literature we have verified that many authors are conducting studies with different materials to improve both prognosis and the success rate of direct pulp coating, such as the MTA, theracal $\AA$, calcium phosphate, Biodentine $\AA$, lasers.

\section{Conclusions}

The aim of endodontics should be the rehabilitation of the disease or necrosis of the dental pulp to normal health and function. It is important to know the properties of the materials, composition and mechanisms of action to facilitate the clinical handling and use. Pulp capping has achieved high rate of success.

\section{- Oral Presentation 28}

TITLE: Effects in predentine of various agents with remineralizing capacity

AUTHORS: Fernández Fernández L, Bolaños Carmona V, Briones Luján MT, Benavides Reyes C.

\section{Objectives}

To test the hypothesis that predentine is a biological substrate sensitive to several materials with remineralizing properties.

\section{Materials and Methods}

Fifteen primary teeth, exfoliated or extracted because orthodontics reasons, were ramdonly assigned to study groups ( $\mathrm{n}=3)$. G1. Control (no treatment); G2, BiodentineTM; G3, OH2Ca; G4, GCTooth Mouse (GCTM); G5, OZn cement (wothout eugenol). Pulp remmanents were hand eliminated. Pulp chamber was backfilled with the material assigned to each study group. Teeth were sealed with composite, then incubated at $37^{\circ} \mathrm{C}$ for 2 weeks. Afterwards, materials were removed and teeth cut in slices $1 \mathrm{~mm}$ thick. Pulp chamber walls and secondary middle crown dentin were analyzed by SEM+EDS or micro-Raman spectroscopy. Comparisons between groups were made using nonparametric statistics. Significance was stablished at a $p$ value lesser than 0.05 .

\section{Results}

The greater modifications in the mineral component of predentin, due to treatment, corresponded to GCTM group, which showed higher values of crystallinity (lesser FWHM) and lesser ratio carbonate/phosphate than the rest of the study groups. GCTM group also had the greater values of the ratio phosphate/CH2 with significant differences respect to the Biodentine ${ }^{\mathrm{TM}}$ group, and the greater relation carbonate/ $\mathrm{CH} 2$ with significant differences respect to control and Biodentine ${ }^{\mathrm{TM}}$ groups. The organic component of predentin did not suffer any significant modifications.

SEM+EDS analysis demonstrated that predentin has lesser percentage of $\mathrm{Ca}$ than dentin. Predentin also showed more frequently than dentin several other elements as $\mathrm{Na}, \mathrm{Zn}$ y $\mathrm{Mg}$ and occasionally $\mathrm{S}$ y $\mathrm{Si}$.

Conclusions

Predentin showed several structural differences with respect to secondary dentin. We detected microchemical and microstructural differences as a result of the contact with some agents with remineralizing properties, especially with GCTM.

\section{- Oral Presentation 29}

TITLE: Pulp Stem Cell Regeneration in necrotic teeth: systematic review

\author{
AUTHORS: Martínez Millán Y, Pérez Guzmán N, \\ Andrada Campillo M, Rodríguez Lozano FJ, Chiva \\ García F.
}

\section{Objectives}

To find out if the procurement of stem cells in daily clinical dental practice for endodontic regeneration would be feasible.

\section{Materials and Methods}

A literature search was conducted in the Medline database of articles published from 2010 to the present. The keywords were: exfoliated tooth stem cells; pulp regeneration; tissue engineering; dental pulp stem cells; endodontic regeneration.

\section{Results}

Endodontic regeneration is based on the triad cells, scaffolds and growth factors. As for possible endodontic scaffolds for regeneration, electrospinning nano- 
fibers have capacity to form the dentin-pulp complex without inflammation. In addition, a three-dimensional structure promotes better scaffolding vascular and cellular organization. Furthermore, cultivation of cellulose spheres stem cells dental papilla allow better regeneration. The phenomenon of storage for cell cryopreservation involves a loss of cell potential, plus additional expenses. Even so, cryopreserved stem cells, specifically autologous, exhibit immunomodulating properties, inhibiting the immune response promoting the regeneration of bone and dental tissues. It should also emphasize the importance of revascularization for endodontic regeneration in necrotic teeth.

\section{Conclusions}

This treatment poses difficulties as cryopreservation, handling and contamination of samples. It is considered a promising technique, but are needed most in vivo studies.

\section{DIAGNOSIS- ANATOMICAL VARIATIONS}

\section{- Oral Presentation 30 \\ TITLE: Cone Beam Computer Tomography (CBCT). Diagnosis of periapical pathology in Endodontics}

\section{AUTHORS: Saralegui Calvo A, González Losada C, Oteo Calatayud J.}

\section{Introduction}

The use of CBCT in Odontology has been expanded to all specialities. In Endodontics its use is also becoming a routine diagnosis procedure.

Tridimensional radiology is the election diagnosis method when clinical symptoms are contradictory, nonspecific or poorly localized. Specially when conventional methods do not provide reliable information or even makes us doubt when making a diagnosis decision.

Its utility would be unquestionable in those clinical situations in which there is a suspicion of periapical pathology on teeth whose canals have previously been treated or not.

\section{Case Report}

We describe clinical cases in which the use of CBCT has been necessary in order to make a diagnosis decision and has been proved to be a more effective method over the conventional ones.

In all these cases the criteria to request a CBCT is: suspicion of periapical pathology with any inespecific clinical symptoms on teeth whose root canals have been into treatment or not. In all these cases we can confirm how clinical synmptomatology does not often match with the poorly conclusive images provided by conventional radiology. Tridimensional radiology has allowed us to be capable of making reliable decisions based on solid image evidence, which apports information of the area explored in the three ortogonal planes.

\section{Conclusions}

CBCT has turned out to be a definitive and reliable diagnosis method for periapical pathology in the studied cases because it has provided additional information in clinical cases when traditional radiographic techniques have not been enough to make a decision on the treatment, mana-ging to employ a mínimum radiation exposure dose.

Small or incipient lessions confined can only be visualized in a conventional periapical radiography when the alveolar labial mask bone has been erosionated or perforated. In these cases tridimensional radiology provides the clinician with a more reliable and earlier diagnosis. 


\section{- Oral Presentation 31 \\ TITLE: Management in the diagnosis and treat- ment of Endo-Perio lesions}

\section{AUTHORS: Goiriz B, Díaz E, Estévez R, Tejedor B,} Cisneros R.

\section{Introduction}

In recent years it has shown that the relationship between periodontal disease and pulp may be sometimes correlated. From the embryological point of view, pulp and periodontal share the same origin so that anatomical and vascular connections are maintained between the two tissues, this being a decisive factor in the origin of injuries endo-perio, also known as combined lesions. Therefore, management in diagnosis and treatment is not always simple. We need to know to make a differential diagnosis with all clinical data obtained from various tests.

\section{Case Report}

We analyze various clinical cases where the injuries described above differ: A combined injury, periodontal injury excluiva state of evolution, a combined lesion whose initial prognosis was doubtful and finally a case of a compatible retreat with a fracture. Through them, we describe exhaustively diagnostic tests to be performed in order to carry out a differential diagnosis.

\section{Conclusions}

Communication between pulp and periodontal tissues may occur through the apical foramen, lateral canals, root fractures or perforations. Lateral canals should be considered means of communication that are able to allow the passage of harmful material or microorganisms in both directions. Thus, the diagnosis of such conditions is vital to carry out a successful treatment and improve prognosis. Note the importance of a good diagnosis to treatment success.

\section{- Oral Presentation 32}

TITLE: Diagnosis value in a suppose root fracture. A case report

\section{AUTHORS: Moquillaza LE, Bruno F, Loroño G, Elzaurdia C, Cisneros R.}

\section{Introduction}

The evaluation of a posible root fracture, usually, is based on subjetive data, the results are not always conclusive; therefore, we are always working on the basis of a suspicion of this. Hence the importance of establishing a multiple evaluation guide of this pathological form, whose early detection has a great importance to assess the prognosis of further treatment.

\section{Case Report}

Attends to clinic a middle-age patient refers pain while biting in 14 piece. It presents slightly positive percussion, a physiological probing and radiographic image evidence is not observed. It was decided to luminosity test in which is seen as the light beam passes completely through the adjacent piece while in the problem piece the light is blocked at the level of the mesial groove. We decided to make the opening of the piece to test staining with methylene blue. After confirmed our suspicion with this luminosity test, staining test, visual test, and medical history of the patient, finally decided to extraction of the piece.

\section{Conclusions}

The root fissures are a pathological form we have to be present in our diagnoses in general, especially in molars and premolars. The evaluation of these injuries will always be multiple, using several tests to get an accurate diagnosis. Once this, the removal of the tooth will be the most common pattern to follow.

\section{- Oral Presentation 33 \\ TITLE: Diagnosis of cemental tear using Cone Beam Computer Tomography}

\section{AUTHORS: Rodríguez Mazón M, Abella Sans F, Garrido Poza I.}

\section{Introduction}

The cementum is a specialized mineralized tissue covering the root surfaces and, occasionally, small portions of the crown of the teeth.

The etiologic factors of disorders of the cementum are divided into categories: congenital, which include cemental dysplasia and grooves on the root surface, and acquired, which include the fractures or tears of the cementum that occur as a complete separation along the cemento-dentinal border,where the fragment is displaced into the periodontal ligament; or partial, where the split occurs within the cementum.

Teeth with cemental tears often present with severe localized periodontal attachment loss, including pocket formation and alveolar bone destruction. There are three ways to confirm a diagnosis of cemental tear: radiographic examination, surgery with direct inspection of the tooth fragment; and histological examination of the removed cemental tear.

The inclusion of cemental tears in the differential diagnostic process of lesions (vertical root fracture, periodontal lesion or a lateral canal) has important clinical implications.

The main objective is the removal of the cementum fragment. This can be achieved by scaling and root planing, with or without periodontal surgery. 


\section{Case Report}

Here, we present a case of cemental tears on the anterior teeth. Two cemental fragments were observed in the vestibular surface of a mandibular central incisor and in the distal surface of a maxillary central incisor. Both cases were treated with periodontal surgery with a full thickness flap. They present a follow-up of 12 months. The teeth are asymptomatic and present radiographic healing.

\section{Conclusions}

Endodontists and dentists may avoid misdiagnosis and unnecessary treatment of teeth with cemental tears if they can properly evaluate the radiographs and pulp vitality of teeth as well as know the predisposing factors and clinical characteristics of teeth with cemental tears in advance.

\section{- Oral Presentation 34}

\section{TITLE: The clinical challenge of the endodontic} treatment of C-Shaped root canal systems

\author{
AUTHORS: Sanchez Dorado G, De Gregorio Gon- \\ zalez C, Aranguren Cangas J, Ceballos Garcia L.
}

\section{Introduction}

The C-Shaped root canal systems are an anatomical variation presented mainly in second mandibular molars. These systems show an isthmus, which represents a clinical challenge during the different phases of the endodontic treatment. The knowledge of these anatomies is essential in the accurate and conservative management of C-Shaped molars.

\section{Case Report}

Two clinical cases are presented, both of them of second mandibular right molars.

The first case is an abutment tooth (47), showing symptomatic pulpitis due to a deep distal decay. The treatments were performed under microscopic magnification, using ultrasonic tips, sonic activation of the irrigants (EDTA 17\% and $\mathrm{NaOCl} 4 \%$ ) using Endoactivator (Dentsply, Tulsa, OK) and vertical condensation for the obturation phase.

The second clinical case (47) was a root canal treated tooth, with asymptomatic apical periodontitis. The obturator material was removed through an R25-Reciproc file (VDW, Munich, Germany), combined with Chloroform as a solvent. A hybrid obturation technique using Gutta-Core (Dentpsly, Tulsa, OK) and thermal compaction is presented, allowing a better penetration into anatomical complexities.

\section{Conclusions}

Given the complexity of these root canal systems, we should not skimp on resources, in order to avoid iatrogenic errors and thus achieve greater success rate. Due to the difficulty in access and instrumentation of these canals, it is crucial to combine the magnification with the use of ultrasonic tips, being more conservative in dentine removal as well. The use of contemporary irrigation and obturation protocols, allow a more predictable microbiological control and sealing.

\section{- Oral Presentation 35 \\ TITLE: Endodontic treatment of 3 roots supe- rior premolar}

AUTHORS: Ávila Castellano A, Ibañez Barranco R, Ibañez Fernandez C, Segura Egea JJ.

\section{Introduction}

The internal anatomy of premolar teeth is very varible. So often, their root canal treatments represent a challenge for the endodontist. Here is reported the case of a second maxillary premolar with 3 roots, 2 vestibular and 1 palatal and 3 root canals. This configuration only occurs in $1 \%$ of the premolar.

\section{Case Report}

A patient seek for treatment by acute pain the left side region of the maxila. Intraoral examination revealed the existence of and oclusal-distal cavity in the root 2.5. Palpation wasnormal. the percusion was positive.

Vitality test provoked a hyper-response. Radiographic examination showed a caries lesion affecting de pulp, with widening of the periodontal ligamnet space. Irreversible pulpitis an apical periodontitis of 2.5 tooth was diagnosed and root canal treatment was prescribed.

after anesthesia and insolation, cavity access carriend out ussing a diamond burr. Regularization of the pulp chamber was performed with an endo $\mathrm{z}$ burr. A thorough exploration of the pulp chamber floor allowed to locate 3 root-canal orifices. Working length was established with an apex locator. Glide path was cariiend out using $\mathrm{K}$ files \# 08-10-15. Protaper next system (files $\mathrm{X} 1$ and $\mathrm{X} 2$ ) was used for root canal intrumentation. Calibration of apical foramen was performed with and \#25 (flexofile) in the vestibular root canal and \#30 in the palatal one. Obturation was carried out using termoplastic gutta percha (Guttacore). Tooth reconstruction was carried with composite A3 (Tetric-evoceram). All treatment was conducted in one sesion.

\section{Conclusions}

Although normally the maxillary second premolar has a single root canal, a further exploration of the pulp chamber floor must be always carried out to detect possible orifices of supplementary root canals. 


\section{- Oral Presentation 36 \\ TITLE: CBT assisted endodontic management of premolars with three roots}

AUTHORS: Sánchez González C, Valencia de Pablo O, Estévez Luaña R, Díaz-Flores V, Cisneros Cabello R.

\section{Introduction}

Variations of root canal systems can become impossible to determine using conventional radiography. Sometimes, the CBCT will allow us to know exactly what we are facing and to develop an individualized treatment plan.

Maxillary and mandibular premolars can have complex anatomies with 3 root canals. In these cases, the magnification and the use of three-dimensional images may be useful.

\section{Case Report}

We report the case of a maxillary second premolar. The initial X-ray did not make us suspect a possible anatomical variation. At a time of permeabilization, a file 10 hooked and radiographically confirmed the presence of a third root canal. A study with CBCT and a microscope were performed, the opening was changed.

The second case is a mandibular first premolar. By initial radiograph we could already suspect the presence of a complex anatomy and, after instrument two main root canals, file 10 snagged in the third. We conducted a CBCT to better plan their clinical approach and under the magnification of the microscope, access was modified to allow treatment.

In both cases, the instrumentation was performed with the file Protaper Next X1 and Race 25.04. Irrigation was sodium hypochlorite $4.25 \%$, final irrigation of $17 \%$ EDTA and hypochlorite again, both activated with Endoactivator. The obturation was vertical condensation, followed by injection of gutta percha.

\section{Conclusions}

Cone beam is an useful treatment to deal with complex cases. It is also a promising diagnostic complement and increases our chances of success in endodontics.

\section{- Oral Presentation 37}

TITLE: Root fusion without concrescence: histological analysis

AUTHORS: Murillo Benítez M, Jiménez Sánchez MC, Tarilonte Delgado ML, Martín Jiménez M, Segura Egea JJ.

\section{Introduction}

Concrescence is a rare dental anomaly defined as the cemental union of two adjacent teeth. This anomaly has been reported with an incidence of $0.8 \%$ in the permanent dentition. Also has been reported a higher incidence of concrescence in the posterior maxilla. Clinically and radiographically is very difficult to detect concrescence, and most of cases are diagnosed post extraction. Concrescence diagnosis requires histological confirmation. A case with clinical diagnosis of concrescence, between a retained third molar and an erupted second molar, where the histological study did not show cementum union between both roots is reported.

\section{Case Report}

A 58-year-old male, seek for dental assistance because of pain in the maxillary right second molar. Diagnosis of irreversible pulpitis was established and root canal treatment was proposed to the patient, who did not accept, preferring dental extraction. During elevation and luxation of the tooth, an abnormal resistance was detected. Finally, the right maxillary second molar was extracted and, unexpectedly, with the third molar still fused to the distal surface of the roots. The first diagnosis was clinical concrescence, however, histopathological study demonstrated that the union between the two root surfaces was through bone and not by cementum.

\section{Conclusions}

Concrescence may occur during root formation (true concrescence) or after the radicular phase of development is completed (acquired concrescence). Space restriction during development, local trauma, excessive occlusal force, local infection after development, play an important etiologic role. The use of cone beam computed tomography (CBCT) could be a powerful supplement that would provide more information to diagnose concrescence. It is necessary to consider the incidence, recognition and implications of this anomaly to develop an appropriate diagnosis and treatment plan. As long as there are no histological confirmation, it is preferable to use the term fused roots, using "concrescence" only after histological confirmation.

\section{- Oral Presentation 38 \\ TITLE: Intentional replantation as treatment of a developmental groove}

\section{AUTHORS: Sansalvador Millet V, Llaquet Pujol M, Duran Sindreu F.}

\section{Introduction}

The developmental groove is a morphological abnormality, also known as a palatal crown- root groove, that can develop the formation of a localized periodontal pocket.

descrien numerous treatments, including elevation of mucoperiosteal flap and rear odontoplasty, and intentional replantation. The latter therapeutic alternative is 
described as tooth extraction, treatment outside the oral cavity, and subsequent reintegration into its socket.

This case shows the treatment of a developmental groove of a lateral incisor diagnosed with asymptomatic apical periodontitis, by subsequent intentional replantation to endodontics, six months control.

The goal of treatment is intentional reimplantation groove development outside the oral cavity, periodontal defect repairing and functional maintenance of the tooth.

\section{Case Report}

On clinical examination, we observed a developmental groove, a borehole located $7 \mathrm{~mm}$. Radiographs showed an apical radiolucency, which was confirmed by the CBCT. We diagnose asymptomatic apical periodontitis.

After the root canal, the intentional reimplantation was performed; We perform the extraction, ensuring minimal damage to the periodontal ligament fibers. With the tooth out of the oral cavity, he was made odontoplasty groove and seal the cavity with flow.

We irrigate during the procedure with skim milk, avoiding keeping the tooth dry.

The tooth is reimplanted in the socket in its original position and sutured with a suture cross, as a splinting for 15 days.

Resolution of the periapical observed at 6 months image control. He showed no symptoms to percussion and palpation, and probing $3 \mathrm{~mm}$.

\section{Conclusions}

The palato-gingival sulcus may cause pulp necrosis and severe periodontal defect.

The treatment performed is highly predictable in such cases, performing: atraumatic extraction, and the time out tooth cavity, continuously irrigating.

\section{- Oral Presentation 39}

\section{TITLE: Clinical management of dens invagina-} tus: report of two clinical cases

\section{AUTHORS: Pressegué Jorba C, Godoy C, Duran- Sindreu F, Abella F, Roig M.}

\section{Introduction}

Dens invaginatus (DI) is a abnormality resulting in a deepening or invagination of the enamel organ into the dental papilla prior to calcification of the dental tissues. Several theories have been presented for explaining the mechanisms behind this malformation and different classification have been used to describe the severity of this abnormality.

The prevalence of DI varies between $0,25 \%$ to $10 \%$ and the permanent lateral incisior are the most frequently affected teeth. Teeth are associated with an increased risk of developing pulpal pathosis without any evidence of caries or trauma.

Identify and study the different types of dens invaginatus by two cases reports.

Determine the correct way to handle the treatment of dens invaginatus.

\section{Case Report}

This case report present two different cases of dens invaginatus type I and type II. The treatment planning, was to diagnose the cause of pulpal necrosis, which was the invagination, the cleaning and shaping of the invagination, and the posterior root canal treatment for both cases, one with gutta-percha and the other an apical plug of MTA.

\section{Conclusions}

After one year of follow up, both cases show a decrease of the initial apical radiolucency,

Dens invaginatus might increased the risk of pulpal pathosis and complicated any root canal treatment.

The use of cone beam computed tomography and magnification can help to manage this dental anomalies.

MTA is an useful material for the treatment of periapical radiolucency having a good prognosis in cases of apexification.

\section{- Oral Presentation 40}

TITLE: Endodontic treatment of a type I dens evaginatus using a guided splints

\section{AUTHORS: Mena Álvarez J, Rico Romano C, Zubi- zarreta Macho A.}

\section{Introduction}

Dens evaginatus is defined as a rare dental malformation. The inherent structural variants of this anatomic disorder make it difficult to perform conventional endodontic treatment procedures by means of a conservative access cavity. In this clinical case report, the authors describe the endodontic treatment of a type I dens evaginatus by means of guided splints for cavity access, and chairside $\mathrm{CAD} / \mathrm{CAM}$ veneer restoration.

\section{Case Report}

This is a case report of a type I dens evaginatus in a maxillary lateral incisor. The authors established the diagnosis by means of cone-beam computed tomography. Information obtained from cone-beam computed tomography allowed the clinician diagnosticate and planning the endodontic treatment of the apical periodontitis. The authors fabricated a splint guide for minimally invasive access opening in this type of case; thus reducing the loss of dental tissue. The authors manufactured a splint guide from a Digital Imaging and Communications in Medicine file (DICOM) and a stereolithography file (STL) obtained from a plaster model of the patient by 


\section{ENDODONTICS COMPLICATIONS- RETREATMENTS} authors obtained a digital impression from an intraoral scan, and fabricated a CAD/CAM veneer through a chairside system, to restore the tooth.

\section{Conclusions}

Cone-beam computed tomography is an effective method for obtaining information about the root canal system in teeth with dens evaginatus. In addition, guided implant surgery software is effective for manufacturing splint guides for endodontic treatment with conservative pulp chamber access.

\section{- Oral Presentation 41 \\ TITLE: CBCT use in the management of a sepa- rated instrument}

\author{
AUTHORS: Santos Montón G, de la Torre de la Fu- \\ ente F, Estévez Luaña R, Tejedor Bautista B, Cisne- \\ ros Cabello $\mathbf{R}$.
}

\section{Introduction}

Separation of an instrument within a root canal during the endodontic treatment is a complication as it hinders the achievement of its objectives: shaping, disinfection and filling. In this situation, we have two different alternatives: attempt to extract the fragment the separate instrument or try to bypass it to recover the patency and make a proper chemical-mechanical cleaning.

\section{Case Report}

A 35-years old woman came to our office sent by another professional because of a separated instrument in the mesiobuccal canal of 46. After a conventional initial diagnosis we decided to take a cone beam tomography. An in-depth evaluation of CBCT allowed us to conclude that the mesiobuccal and mesiolingual canals were connectted apically to the point where he had the separated instrument. Based on this information we shaped the mesiolingual canal mechanically and we cleaned the mesiobuccal canal chemically. Root canals were instrumented using Wave One Primary (Maillefer, Ballaigues, Suiza). 4,2\% sodium hypochlorite and 17\% EDTA were used as irrigating solutions agitated by pasive ultrasonic irrigation. After the three-dimensional sealing by warm vertical compaction we could see as only an appropiate irrigation allows the sealer to slide through the separate instrument to the mesiolingual canal. This fact avoids removing dentin from the walls of the canal with the fractured instrument that can weaken the tooth to the occlusal loads.

\section{Conclusions}

Cone beam tomography gives us a three-dimensional image of the tooth to be treated as we can see exactly the area where the separation has occurred and the anatomy of the canal. This three-dimensional information can be very useful to deal with the handling of the case.

\section{- Oral Presentation 42 \\ TITLE: Clinical management of a root perfora- tion in an inferior first molar}




\section{AUTHORS: D'Amico G, Elzaurdia C, Loroño G, Es- tevez R, Cisneros R.}

\section{Introduction}

According to the consulted literature, perforation incidence during endodontic treatment ranges from $2.3 \%$ to $12 \%$. Furthermore over $50 \%$ of perforations occur in lower molar teeth. A perforation or a communication between the inner part of a tooth and the periapical tissues can lead to inflammation and the destruction of periodontal fibres and alveolar bone thus causing a periodontal defect. It is important to diagnose and repair perforations as soon as possible.

\section{Case Report}

A female patient, whose medical history had no relevant contribution, came to our office complaining about pain in the mandibular left side. We get a negative result from a pulp vitality test of tooth 36 and pain when vertically percussion. Diagnosis: pulp necrosis with apical severe periodontitis. After opening the tooth and trying to remove the pulp from the radicular canals, a perforation in the distal part of the mesiovestibular canal is accidentally caused; for this reason the patient is referred to the Department of Endodontics. After complete isolation with rubber dam, the sealing with MTA is carried out, obstructing the MV y ML canals with gutta-percha so as to avoid that MTA might fall into the canals during the sealing of the perforation. Two cones of gutta-percha are left inside the mesial canals and a sterile wet cotton sponge is left on the MTA for one week. During the following clinic session we take a control radiography to check the MTA stability, we carry out the instrumentation (WaveOne Gold), the irrigation ( $\mathrm{NaOCl} 5.25 \%+$ EDTA 17\%) and the sealing (thermoplastic gutta-percha withSystem B) of the root system of tooth 36 .

\section{Conclusions}

During following check up the patient is completely asymptomatic. A prompt diagnosis and intervention, for a perforation, are recommended in order to have a favourable prognosis.

\section{- Oral Presentation 43}

TITLE: Management of root perforations: conserve or extract?

\section{AUTHORS: Encinas M, Durán-Sindreu F, García M, Olivieri G.}

\section{Introduction}

Repair of perforations poses a challenge due to different factors such as visualization and access, bleeding, localization, size, time and material used.

The appearance of MTA is a turning point in the prognosis of repairing teeth with perforations, because of its many beneficial properties that indicate it as the ideal material for sealing perforations, achieving a better prognosis than the obtained with materials used in the past, as amalgam or gutta percha.

\section{Case Report}

We present two clinical cases of sealed perforations with MTA with healing observed in controls after evaluating the factors affecting the prognosis according to classical and modern literature.

\section{Conclusions}

The success rates achieved in the management of teeth with perforations according to modern literature, indicate that orthograde repair of perforations using MTA is the right and appropriate treatment in all areas of the root.

\section{- Oral Presentation 44 \\ TITLE: Overextension and overfilling in apical periodontitis}

\section{AUTHORS: Fernández Cartusciello J.}

\section{Introduction}

In apical periodontitis cases, the main factor is the intraradicular infection; therefore endodontic procedures must focus not only in preventing the introduction of new microorganisms in the root canal system, but also in removing them.

The procedures inevitably cause some damage to the periapical tissues. The ultimate goal is to make the canal space impermeable to leakage and microbial recontamination.

When we talk about overextension is referred to an extensive preparation of making the sealing material go beyond the apical foramen and we talk of overfilling, it is when we have an apical stop in the preparation but there is a cement extrusion beyond the apical foramen.

Case Report

4 clinical cases are described with apical periodontitis, where there have been over extension and overfilling material when performing endodontic treatment. A control at 6 months and a year are performed to determine if there have been signs of healing during this period.

\section{Conclusions}

In the 4 clinical cases healing was observed after 6 months and a year, this means that the sealing material beyond the apical foramen does not influence over the healing of periapical tissues.

It is more important to make a good preparation biomechanics disinfection in the fact that the material is beyond the apical foramen

These tissue injuries caused by filling materials either gutapercha or cement extruded is usually transient, but not persistent. 


\section{- Oral Presentation 45 \\ TITLE: Exacerbations of pulp pathology: a case report}

\section{AUTHORS: Cardona Barrachina R.}

\begin{abstract}
Introduction
A flare up is a complication of endodontic treatment, defined as an acute exacerbation of asymptomatic pulp or periradicular pathology after initiation or continuation of root canal treatment. Other features include the onset of pain and / or swelling during endodontic treatment that requires active interference of the dentist.
\end{abstract}

\section{Case Report}

Came up to the university hospital a patient of 41 years old, with no medical history of interest. The complaint was that the patient wanted to change the crown for aesthetic reasons because at age 12 had a trauma. Clinically he had a screened in tooth 2.1, asymptomatic horizontal, vertical percussion and palpation crown. Probing was within physiological parameters. He presented radiographically open apex with periapical image and crown post without endodontic treatment performed. It was explain to the patient different treatment options. Finally, apexification and metal-ceramic crown is made. Between visits, after starting the endodontic treatment, th patient came presenting emergency swelling in the area and symptoms to percussion and palpation. It was prescribed antibiotic and anti-inflammatory.

Following this event a literature review was performed to clarify witch preventive measures can be used in these situations in order to obtain a protocol.

\section{Conclusions}

Because microorganisms are the main causative agents of these events, knowledge about microbial mechanisms involved in the etiology is paramount. Therefore, appropriate guidelines should be follow to avoid an emergency, in between visits, with acute pain and / or inflammation. It has been shown that a flare up has no significant influence on the prognosis of endodontic treatment, its appearance is undesirable for the patient and dentist.

\section{- Oral Presentation 46}

TITLE: Difficulties encountered in a retreatment. A case report

AUTHORS: Conde Villar AJ, Estévez Luaña R, Martín Escorial A, De la Torre De la Fuente F, Cisneros Cabello R.

\section{Introduction}

One of the most common causes of endodontic failure is the presence of bacteria inside the canal system due to a poor seal. Retreatment present many difficulties by multiple factors such as alteration of the original anatomy, filling materials and in this case, also coronal obstructions.

\section{Case Report}

A patient was remitted to the Postgraduate Program in Endodontics for root canal retreatment on the tooth 36 . After radiologic examination and supplementary tests the established diagnosis was chronic apical periodontitis by previous endodontic treatment failure. We use microscope and ultrasound to remove the post in distal root, and thus proceed to remove contaminated gutta percha. Once achieved, precurved hand files to bypass the blocks located in the apical third. An omitted canal was located. Glide path was performed with Path File and root canal instrumentation with Mwto and Profile 30.06. Final irrigation protocol with $\mathrm{NaOCl} 5.25 \%+$ $17 \%$ + EDTA $5.25 \% \mathrm{NaOCl}$ Endoactivator sonic activation were used. Filled with Autofit $6 \%+$ accessory points, down-pack with alpha-endo unit and backfill with endo-beta gun.

\section{Conclusions}

Before endodontic failure we always consider nonsurgical retreatment, despite the difficulties. Microscope and ultrasonic devices are recomended to manage root canal retreatment.

\section{- Oral Presentation 47 \\ TITLE: Endodontic treatment of an inferior premolar with a full-coverage crown and root canal sclerosis}

\section{AUTHORS: Ruiz Japón P.}

\section{Introduction}

The presence of full-coverage crowns raise the root canal treatment difficulty. The lack of visibility of the pulp chamber and the infrequent coincidence of the center of the crown with the center of the stump to treat increase the possibility that an accident occur. A root canal treatment of a second inferior premolar with a full-coverage crown and root canal sclerosis is described.

\section{Case Report}

A 53-years-old-woman showed pain to cold and hot stimu-lus in the tooth 4.5 , restored with a full-coverage crown that was joined with the teeth 4.4 and 4.6 , being the 4.6 an implant-supported crown. Clinical exploration showed lightly positive palpation and percussion in the tooth 4.5, with a physiological periodontal sounding. Radiographic exploration revealed that its inner plate was blurred in the periapical region as well as a very narrow root canal. A diagnosis of irreversible pulpitis and symptomatic apical periodontitis of the tooth 4.5 was established, and the root canal treatment was prescribed. The patient did not let us remove the metal- 
ceramic bridge, so the root canal treatment of the tooth 4.5 was carried out through the crown. By using EDTA $17 \%$ and a $0,13 \mathrm{~mm}$ PathFile file, glide path and patency could be managed. The canal was de-brided until the 25/06 MTwo file. While the 20/05 MTwo file was being used, a ledge formation made necessary to perform a recapitulation with hand files before to use the 25/06 MTwo file. The obturation was carried out by using the continuous wave technique (B\&L Biotech ${ }^{\circledR}$ system), preventing the leakage of the endodontic sealer because of the mental foramen proximity.

\section{Conclusions}

The presence of full-coverage crowns and physiological complications like the root canal sclerosis make the root canal treatment more difficult, but do not prevent it. The treatment can be performed by using suitable tools and materials.

\section{- Oral Presentation 48}

\section{TITLE: Treatment of a pulpar bleeding in a} central incisor after an extrusive luxation

\section{AUTHORS: Fernández Prieto C, Martín Jiménez M, Sánchez Blanco I, Segura Egea JJ.}

\section{Introduction}

Extrusive luxation is a traumatic damage of the tooth supporting tissues which leads to a break of the periodontal ligament fibers and as a result a displacement of the tooth axially out of the socket. Pulp vitality and aesthetics of these teeth can be compromised.

\section{Case Report}

A 28-years-old male patient suffered an assault during the orthodontic treatment. He came to our dental clinic three weeks after the trauma. At clinical examination, 2.1 had an extrusive displacement of about 4-5 $\mathrm{mm}$ and that position had been maintained thank to the orthodontic arch and the absence of occlusal interferences. Pulp vitality was not conserved and there was a severe tooth discoloration. At the X-ray examination widening of periodontal ligament was observed. Due to the time elapsed since the accident; the tooth could not be repositioned manually. Root and canal treatment was done in a single session, afterwards three sessions of internal bleaching with sodium perborate were done and orthodontic treatment was resumed after two months. The tooth was replaced in its position by orthodontic intrusion and treatment was successfully completed.

\section{Conclusions}

There is no evidence of contraindication for orthodontic treatment after a dental trauma, however depending on the type of damage on the tooth and periodontal ligament we must establish observations periods prior to the application of orthodontic forces.

\section{- Oral Presentation 49}

TITLE: Management of complicated crown fractures in permanent dentition with fragment reattachment

\author{
AUTHORS: Ruiz XF, Dávalos M, Mercadé M, Abe- \\ lla F, Durán-Sindreu F, Roig M.
}

\section{Introduction}

Crown fractures represent one of the most common dental traumas among permanent dentition in children and teenagers. The upper incisors are the most frequently affected teeth.

When a complicated crown fracture ocurrs (with pulp exposure) in permanent teeth, the main goal is to preserve pulp vitality in both closed apex teeth and inmature teeth. The irreversible affection of the pulp will imply thin dentin walls and an incomplete development of the root, therefore increasing the risk of fracture.

Direct pulp capping and partial pulpotomy were proposed by the International Association of Dental Traumatology with high success rates.

According to the restorative treatment, different techniques have been proposed to treat crown fractures when the fractured fragment is present. Adhesive reposition of the fragment represents a minimal invasive technique with a great aesthetic result and high success rates, thanks to the advance in adhesive restorative dentistry.

\section{Case Report}

Two clinical cases diagnosed as complicated crown fractures treated with vital pulp preservation (partial pulpotomy with BiodentineTM ) and fragment repositioning with an adhesive tecnique are presented. The first case shows a crown fracture in a closed apex permanent tooth and the second case in an inmature tooth.

\section{Conclusions}

Complicated crown fractures in permanent dentition imply a great challenge for the practitioner. The main goal lies in vital pulp preservation, aesthetics and function in those patients, and furthermore to assure the survival of these teeth as much as possible.

Nowadays, tooth fragment reattachment represents a conservative treatment, that guarantees the success of those traumatized teeth in young patients.

\section{- Oral Presentation 50 \\ TITLE: Bleaching of non-vital teeth to remove intrinsic stains}

AUTHORS: Sánchez Durán D, Benavides Reyes C, Cabello I. 


\section{Introduction}

Tooth bleaching presents great importance in dental esthetics. In discolored non-vital teeth, the intracoronal bleaching procedure is widely used because it is efficient, relatively simple, presents low cost and preserves the dental hard tissue compared to the prosthetic treatment. Intrinsic discoloration of non-vital teeth may have a significant esthetic and social impact on adolescents. When this involves discolored endodontically treated teeth, consensus can hardly ever be found as to the chosen approach. Several bleaching techniques and materials have been proposed in order to obtain satisfactory bleaching and no damages in dental hard tissues and periodontal ligament: bleaching, crowns or veneers. However, this restorative crown or veneer approach has a significant drawback of being an invasive technique. Among bleaching techniques, the walking bleach technique stands out because of its superior esthetic results with no side effects. This work presents several case of tooth discoloration in non-vital tooth which were successfully bleached using walking bleach method.

\section{Case Report}

Nonvital, discolored teeth were bleached by sodium perborate mixed with distilled water. A glass ionomer cement was used as a mechanical barrier in an attempt to minimize the undesirable side effects of intracoronal bleaching. After seven days bleaching agent placement, treatment effectiveness was assessed, and perborate was removed or substituted in case of being necessary. Bleaching agent replacement was necessary in most cases (usually twice for each treatment).

\section{Conclusions}

Walking bleach technique is easy to perform and has a high efficacy; high level of patient satisfaction after non-vital tooth bleaching treatment strongly supports this treatment modality to be a conservative treatment alternative for traumatized discolored anterior tooth or before a multidisciplinary treatment.

\section{- Oral Presentation 51 TITLE: Post removal: fiberglass post vs. metal post}

\section{AUTHORS: Villarroel G.}

\section{Introduction}

In nonsurgical retreatment, the presence of a post at the root is an obstacle that must be removed in order to be able to carry out the treatment. In a study of 2000 patients treated in endodontic practice, Abbott (1994) reported that $36.7 \%$ of these cases underwent retreatment and $25.7 \%$ of those required post removal. This represents $9.4 \%$ of all cases treated and demonstrated the need to be competent when removing posts in atraumatic way to prevent vertical cracks or fractures.
Different techniques have been proposed for this purpose. Including the use of ultrasound. However the degree of difficulty may vary depending on the type of post.

Techniques used and possible present difficulties in removing fiberglass and metal post through two cases are described.

\section{Case Report}

Two cases are described. The first is a tooth 45 , requiring retreatment and with a fiberglass post, microscope and diamond burs where used for removal. The second case, consists of a tooth 46 , with symptomatic apical periodontitis and had a metal pin at the distal root. Microscope and ultrasound was used for removal.

\section{Conclusions}

Because the translucency of fibers glass post can be more difficult and risky to be removed when copared with metal post.

Studies comparing two techniques for removing fiber posts, concluded that the removal using the combination of Long and diamond burs is the fastest and most effective technique.

It is essential to use magnification to avoid unnecessary tissue removal.

The removal of metallic prefabricated post with ultrasound has proven effective. Studies show that the type of cement does not influence the force required to remove the post.

\section{- Oral Presentation 52 \\ TITLE: Cone Beam Computer Tomography (CBCT). Canals retreatments based of diagno- sis evidences}

\section{AUTHORS: González Losada C, Saralegui Calvo A, Saralegui González P.}

\section{Introduction}

The use of CBCT in Odontology has been extended to all the specialities. In the discipline of Endodontics its utility has been proved when confirming a suspected diagnosis in complex clinical cases. It can also be employed as an intraoperatory tool which provides us with valuable additional tridimensional information.

Cone Beam Tridimensional Radiology is the election diagnosis method when clinical signs do not provide enough reliable information in order to take a diagnosis decision or makes us doubt.

Root canal retreatments in Endodontics are clinical situations which require additional information, to improve our options for success.

\section{Case Report}

We expose a series of clinical cases in which CBCT has been necessary in order to make a diagnostic and 
therapeutic decision. In all of the mentioned cases we provide these tridimensional images which have been fundamental for us to stablish the cause of the previous canal treatment's failure.

In all these clinical cases the criteria which has brought us to request a tridimensional radiology have been the need to carry out a canal retreatment and to discard the existence of other dental pathologies which might have contraindicated this procedure. Besides, in all of the described cases conventional radiologic methods have been proved to be insufficient for the clinician to make a solid diagnosis relying on the ALARA (As Low As Reasonably Achieveable) criteria.

We show clinical cases in which CBCT has been a conclusive evidence to decide wether root canal retreatment is the best way to proceed or not. This technology provides the clinician with additional information about the existence of defectively sealed canals or the presence of an additional one, circumstances which can be solved with a root canal retreatment. In addition, CBCT is a really helpful tool to identify any perforations, fissures or fractured roots, as well as the exitence of fractured instruments which contraindicate any canal retreatment.

\section{Conclusions}

In all the clinical cases described before, $\mathrm{CBCT}$ has been definitive and indispensable to confirm the diagnosis and to help the clinician when making the decission to either practice a root canal retreatment or not. Nowadays, CBCT is becoming an essential diagnostic technique in Endodontics since it allows the clinicial to confront difficult clinical cases in which additional information might be required to make a more reliable treatment choice and to improve the patient's prognosis.

\section{- Oral Presentation 53}

TITLE: Multidisciplinary treatment of cystic lesion periapical for endodontic failure. About a clinical case

\section{AUTHORS: Navajas Nieto JM, Navajas Nieto C.}

\section{Introduction}

One of the failures of endodontic treatments is the permanence or new aparción of periapical lesions that require complex surgical treatment.

\section{Case Report}

We present the treatment of a 37-year-old who suffers recurrent exacerbation of a periapical lesion in the tooth 2.1., Teeth were treated in childhood, after trauma, through endodontics, reconstruction with bolts and metal crowns ceramics.

After his diagnosis follows: cystic enucleation, tissue regeneration using xenograft bone block and der- mal membrane, alveolar closure by pedicled autograft iirigación, implant, orthodontics and aesthetics of their crowns replacement.

\section{Conclusions}

Nowadays, dental treatments are usually multidisciplinary. The presence of several specialists in the dental clinic, is absolutely necessary for the benefit of the patient.

\section{- Oral Presentation 54 \\ TITLE: Retreatment in a single sesión of a supe- rior incisor with acute apical abscess}

\author{
AUTHORS: Barrientos Delgado JF, Yanes Barroso \\ E, Ibáñez Barroso R, Montero Miralles P, Segura \\ Egea JJ.
}

\section{Introduction}

One of the most common endodontic emergencies is acute apical abscess. In root-filled tooth, acute apical abscess is a flare-up of a persistent chronic apical periodontitis. There is no consensus in the scientific literature about if, in these cases, the retreatment should be carried out in one or in several sessions. A case of successful endodontic retreatment performed in one session in an upper lateral incisor with acute apical abscess is reported.

\section{Case Report}

A 22 year old male patient, who seek for urgent dental care because intense pain and slight swelling in the region of the canine fossa of the second quadrant. Radiographic examination showed that the tooth has been endodontically treated, showing a wide periapical radiolucent lesion. After establishing the diagnosis of flare-up of persistent chronic apical periodontitis, we proceeded to do endodontic retreatment, appearing abundant suppuration the cavity access. It is carried out with rotary instrumentation crown-down technique by using Protaper Universal files. Once suppuration ceased, abundant irrigation with $5.25 \%$ sodium hypochlorite was performed, sealing with gutta-percha Autofit and AH Plus root canal sealing material, using obturation continuous wave with B\&L System and finishing the retreatment in one session. Follow-up at 6 months shows the favorable evolution of the periapical lesion.

\section{Conclusions}

In cases of acute apical abscess in root-filled teeth, there is scientific literature recommending that, when there is suppuration, the retreatment was carried out in several sessions, putting intracanal medication. However, the outcome of this case suggests that, after suppuration has stopped and after a rigorous disinfection and irrigation of the root canal system, treatment in a single session can also be successful. 


\section{- Oral Presentation 55 \\ TITLE: Endodontic retreatment of a maxillary lateral incisor with two roots. Clinical case}

AUTHORS: Sepulveda-Tendillo S, Teruel-Rodríguez A, Vázquez-Natividad I, Sais-Sais J, Fayos-Soler T, Faus-Llácer VJ.

\section{Introduction}

As traditionally described in the literature the maxillary lateral incisor is a single-rooted tooth with a single canal. However, unusual morphology of its root canal system has been reported. It is essential the professional's knowledge of the anatomy of the root canal system and its possible variations since a major cause of the failure of a root canal treatment is the presence of not treated canals. The use of operating microscope, conebeam computerized tomography (CBCT) and ultrasonic instruments result in more predictable and successful endodontic outcomes. This case report describe the endodontic retreatment of a maxillary lateral incisor with two roots and two canals.

\section{Case Report}

A 70-years-old patient attended to our department concerned about the mobility of her left lateral incisor. Clinically tooth 2.2 showed mobillity grade II, periodontal probing of $11 \mathrm{~mm}$ in palatal and interproximal regions, and tender to vertical percussion. CBCT showed an endodontically treated root and an extra root not treated, both associated to a big apical lesion. Retreatment was performed using Protaper Universal Retreatment System with operating microscope and ultrasounds. Both canal were obturated with thermoplastic gutta-percha. A fiber post was placed in the vestibular canal to perform the tooth restoration. Monitoring during 6 months showed a slow but positive trend with reduced apical lesion and the initial periodontal probing.

\section{Conclusions}

At present, technological advances applied to Endodontics facilitate the treatment of complex cases; while CBCT provides an indispensable diagnostic information, the use of magnification with operating microscope and the use of ultrasonic devices allow a minimally invasive approach.

\section{- Oral Presentation 56}

TITLE: Orthograde canal retreatment (1.6). Complex case of a patient with bisphosphonate treatment

\author{
AUTHORS: Ibáñez Barranco R, Ibáñez Fernández \\ C, Zabalegui Andonegui B, Segura Egea JJ.
}

\section{Introduction}

Ostheoporosis is the common osseous disease and it caused by alterations on the bone replacement mechanisms. Nowadays, biphosphonates are the indicated treatment for it. However, several post-extraction ostheonechrosis-related complicationshave been reported on long time period biphosphonates medicated patients undergoing ostheoporosis process.

\section{Case Report}

A female patient was referred to the practice to have root canal retreatment on the upper right first molar. The tooth was abutment to a fixed bridge and presented a separated instrument inside. The patient had been taking biphosphonates (Actonel $75 \mathrm{mg}$ ) for the past 5 years in order to treat and prevent osteoporosis. After a propper examination, acute periapical periodontitis was diagnosed and a separated instrument was confirmed to be located inside the messio-buccal root. A CBCT was taken in order to obtain as much information as possible and external root resorption was also observed and diagnosed. Biphosphonate treatment was not discontinued. Fixed bridge was removed, a post was also removed using ultrasonic vibration and separated instrument was by-passed with hand-files. Thermoplastic inyected gutta-percha was used to fill the canals. All this was assisted by the use of operatory microscope. After one year the patient is asymptomatic and healing process can be reported keeping the tooth's function as an abutment and bridge's function and aesthetics.

\section{Conclusions}

The use of the CBCT allows us to easily observe and diagnose several pathologies such as external root resorption.

This becomes more difficult when we only work twodimension radiographs. Magnification is essential when trying to remove or by-pass a separated instrument inside a narrow canal such as the one in this case. Orthograde root canal retreatment is totally indicated in this case as tooth extraction involves a high risk of developing ostheonechrosis and the loss of the fixed bridge. 


\section{REABSORPTIONS}

\section{- Oral Presentation 57 \\ TITLE: Treatment of external cervical resorp- tion class II of Heithersay}

\author{
AUTHORS: Loring Castillo A, Casas Rivera A, Es- \\ corial A, Estévez Luaña R, Cisneros $R$.
}

\section{Introduction}

Root resorption is classified into internal and external depending on their location on the root surface. Among external cervical resorption(ECR) we can find the invasive one, involving the progressive loss of cement, dentin and enamel by odontoclastic action, with substitution of fibrovascular tissue derived from periodontal ligament. It is presented below the epithelial attachment in the cervical region and is characteristic the presence of a "pink spot".

Its etiology is due to damage or loss of the protective layer of cementum and consequent periodontal ligament irritation. The most commonly associated predisposing factors in the etiology of ECR are orthodontic treatments, dental trauma and internal bleaching. Heithersay classified ECR according to the extend of the lesión within the tooth in 4 classes.

\section{Case Report}

We report a case of a 12 years old male patient treated with orthodontics and referred to the Master of Advanced Endodontics of the European University of Madrid by the presence of a "pink spot" on the upper right lateral incisor. He had negative palpation and percussion, increased sensitivity, physiological movility and $5 \mathrm{~mm}$ in vestibular probing. It was diagnosed as invasive cervical resorption class II of Heithersay and irreversible pulpitis.

A combined treatment of endodontic and orthodontic extrusion was planned, but finally it was not necessary. Therefore, we performed root canal treatment with rotary technique in two sessions, placing calcium hydroxide between them and it was obturated by warm vertical condensation. We continued with the complete removal of the resorptive tissue and restoring the resulting defect with Relyx Unicem (3M-ESPE). Three months later an esthetic composite restoration was done and after 6 months the revision has been successful, with no symptoms and reducing probing to phisiologic.

\section{Conclusions}

Early detection is essential for successful treatment. Patients with a history of one or more predisposing factors should be monitored for early signs of resorption.

\section{- Oral Presentation 58}

TITLE: Direct pulp capping of an invasive cervical resorption with Biodentine (Septodont)

\author{
AUTHORS: Barrenechea S, Estevez R, De la Torre \\ F, Valencia O, Cisneros R.
}

\section{Introduction}

Invasive cervical resorption is a type of inflammatory external resorption with unknown aetiology. Characterized by a subgingival location in the cement enamel junction. Early diagnosis and proper care are the key to a successful outcome and better prognosis.

Biodentine (Septodont) is an art material, bioactive dentin substitute, has excellent sealing properties with benefits such as preservation of pulp vitality.

\section{Case Report}

50 year old patient comes to the clinic for a periodontal evaluation. After radiographic examination diagnosed a case of invasive cervical resorption in the lower left canine (33).

It responded positively to sensitivity tests.

It was diagnosed as invasive cervical resorption, Heithersay class II.

The surgical procedure was performed under troncular mandibular infiltration with articaine with epinephrine $1.0 \mathrm{mg}$, the intrasulcular mucoperiosteal flap was raised lingual access. $2 \mathrm{~mm}$ osteotomy was performed. Following the suggestion of the cameral pulp through the thin layer of healthy dentin remnant, we decided to make a direct pulp capping with tricalcium silicate (Biodentine, Septodont) Onceset, polish and repositioned flap suture non-absorbable monofilament $5 / 0$ and double points.

The sutures were removed after 7 days, the patient was asymptomatic.

We present radiograph a week, a month and at 3 months. The piece is completely asymptomatic and positive vitality.

\section{Conclusions}

Biodentine (Septodont) is a biocompatible and bioactive material of last generation, which promotes remineralization and healing of the pulp, thus makes it ideal material for this case reconstruction.

\section{- Oral Presentation 59}

TITLE: Treatment of an external cervical resorption with Biodentine

\section{AUTHORS: Artieda Estanga A, Rivas Mundiña B,} Bahillo Varela M, Martín Cruces J, Castelo Baz P.

\section{Introduction}

External cervical resorption is the loss of dental hard tissue as a result of odontoclastic action. It is of non-pul- 
pal etiology and usually begins on the cervical region of the root surface of the teeth by late inflammatory process of the radicular cement, thus making it difficult to diagnose. Treatment depends on the severity, location, whether the defect has perforated the root canal system, and the restorability of the tooth. Early detection is essential for the successful management of ECR as smaller lesions offer the most favourable long-term outcome. MTA is one of the most successfully used materials in these type of clinical procedures but several new materials like Biodentine have recently been developed in response to the growing demand to overcome its prolonged setting time and the potential for discoloration.

\section{Case Report}

46 year-old female patient attends the appointment due to constant throbbing pain on UR8. A radiolucent lesion was discovered in the buccal cervical region of the upper right third molar on radiographic examination with lasting and exacerbating response to vitality testing. Heithersay type II-III external cervical resorption was diagnosed. Root canal treatment was performed and the resorptive tissue was completelly removed with the aid of trichloroacetic acid. Finally, resorption area was filled using Biodentine.

\section{Conclusions}

Biodentine has recently became an excellent alternative to MTA because its setting time is shorter and does not cause discoloration. It is used in a variety of treatments including external cervical resorption.

\section{- Oral Presentation 60 \\ TITLE: How to manage a double internal root resorption}

\section{AUTHORS: Domínguez Marsal B, Sierra Lorenzo A, Tejedor B, Estevez R, Cisneros R.}

\section{Introduction}

Internal inflammatory root resorption destroys dental hard tissue by odontoclast activity. Internal resorption starts inside the root canal and requires at least partially vital pulp tissue. If the resorption is not detected and remains untreated, it can grow larger and perforate the root. When internal resorption is detected early enough, the treatment is usually successful, and the long-term prognosis is good.

\section{Case Report}

A 45-year-old man was referred because of a resorptive lesion in the root of the upper left central incisor, which was discovered thanks to a periapical radiograph. The periodontal condition was excellent, positive percussion and all teeth in this quadrant responded normally to cold testing.
Radiographic examination revealed a well-circumscribed, fairly oval radiolucency in the coronal part of canal. It was neccesarry to do a CBCT to asess the final diagnostic. We concluded with 3D findings that tooth 21 have not perforating internal resorption and root canal therapy was initiated. The tooth was isolated and, after, the cavity access was done and the working length was measured. Root canal instrumentation with Protaper Next (Denstply). Intracanal irrigation was done with $5.25 \%$ sodium hypochlorite after each instrument, and a final irrigation sequencie: Sodium hypochlorite, EDTA and hypoclorite, with sonic activation. The warm vertical compaction was used to obturate the canal, with de B\&L system.

After 12 months, the tooth was asymptomatic and the periapical lesion had healed.

\section{Conclusions}

The use of CBCT helped us to make a correct diagnostic and appropriate treatment plan, because it provided us a 3-dimensional image to appreciate the tooth and injury 


\section{INMATURE PERMANENT TEETH}

\section{- Oral Presentation 61 \\ TITLE: Revascularization of inmature perma- nent teeth}

\author{
AUTHORS: Aboy Pazos S, Castelo Baz P, Martín \\ Biedma B, Ruíz Piñón M, Varela Patiño P.
}

\section{Introduction}

Treatment of permanent teeth with open apex is a challenging procedure for the endodontist because of its complexity of execution. Pulp necrosis of these teeth cause stop the root development which causes the alteration of the crown-root ratio, thin dentinal walls and consequently an increased risk of fracture. One possible treatments for inmature teeth with pulp necrosis is pulp regeneration, which main objectives are the elimination of clinical signs and symptoms, the resolution of the apical periodontitis and the continuation of the root formation.

\section{Case Report}

Woman, 10 years old, comes in with vestibular abscess and fistula with pulp necrosis in 4.5 .

Treatment is planned in two sessions. In the first, the access cavity is made, it is irrigated with sodium hypochlorite $1 \%$ and biantibiotic paste (mixture of metronidazole and ciprofloxacin) is placed. The cavity is sealed with a temporary restoration (Cavit). In the second session it held at two weeks after the first, the root canal is irrigated with $17 \%$ EDTA and bleeding is induced with a K-file. The root canal is dried and place a collagen sponge (Collacote) before the placing of a barrier of 3-4 mm of MTA (ProRoot) in the coronal third of the root canal. The final restoration is done with composite (Ceram X Duo).

At follow-up visits a favorable evolutions are seen, such as a disappearance of clinical symptoms, increase in the thickness of the root walls and as an increased root length.

\section{Conclusions}

The pulp regeneration is an excellent alternative to apexification with silicate (MTA or Biodentine) when treating immature teeth with open apex.

\section{- Oral Presentation 62}

TITLE: Treatment of inmature permanent teeth: a challenge for the clinician

\author{
AUTHORS: Palma B, Escorial A, Díaz-Flores V, \\ Tejedor B, Cisneros R.
}

\section{Introduction}

Disruption of root development by trauma is a very common problem. The main problem that is presented to us is the lack of root development which makes the tooth more susceptible to fracture. Treatment of these teeth is a challenge for the endodontist.

\section{Case Report}

Patient goes Endodontics Program at the Universidad Europea de Madrid presenting pain in tooth 21. Parents say that four years ago had taken a blow and had rebuilt the tooth. After examination and complementary tests, we observe an apical lesion on tooth 21 , as well as presenting an apex of high diameter. As a treatment, we decided to make a apexification. The treatment was performed in three sessions. In first appointment, after opening and cleaning the canal with $4,25 \%$ sodium hypochlorite, we determine the working lenght and made a $4 \mathrm{~mm}$ apical MTA plug. In second appointment, we perform the root canal filling using thermoplastic guttapercha and technical vertical condensation. Finally, at the third session, we made the final reconstruction of 21 with composite resins. At 6 months follow-up the patient presents without symptoms. Radiographically we observe decreasement of the apical focus.

\section{Conclusions}

The use of MTA helps us creating an apical barrier preventing bacterial filtration, which represents a breakthrough in the treatment of these permanent teeth with immature apex.

\section{- Oral Presentation 63 \\ TITLE: Conservative multidisciplinary treat- ment of a traumatized anterior tooth}

\author{
AUTHORS: Della Nave P, Lozano Serrano E, Aiuto \\ R, Mellado Valero A, Mañes Ferrer JF.
}

\section{Introduction}

The preservation of the natural dentition, restoring lost aesthetics and function, is possible by performing a multidisciplinary treatment, involving either ultraconservative restorative and endodontic therapies or minimally invasive surgeries. The aim of this work was the multidisciplinary conservative treatment of a traumatized open-apex central incisor.

\section{Case Report}

A male patient, aged forty, attended our Dental University Clinic (European University of Valencia) for the assessment of a fistula in the tooth 1.1, which he related with a traumatic antecedent. The clinical examination showed a fracture of mesioincisal angle and discoloration. In the complementary radiographic examination, we observed a periapical radiolucent area, associated with that tooth, and an open apex. The therapeutic plan 
began with endodontic treatment. Once solved the fistula, a mineral trioxide aggregate apical stop was created in order to carry out the canal filling using gutta-percha vertical condensation technique. Given the radiological persistence of the lesion, nine months after the beginning of the endodontic therapy, we performed an apicoectomy, whose histopathological analysis confirmed the presence of a radicular inflammatory cyst. In the follow-up x-ray (6 months), the final healing of the lesion could be observed. Subsequently, we treated the discoloration though a non-vital bleaching with hydrogen peroxide $37 \%$, and concluded treatment with the reconstruction of the incisor with composite resins.

\section{Conclusions}

After an accurate diagnosis and thanks to a multidisciplinary and conservative approach, it was possible to maintain the tooth, restore its aesthetics and function, and cure the inflammatory periapical pathology.

\section{- Oral Presentation 64 \\ TITLE: Regeneración pulpar como alternativa al tratamiento en dientes con ápice inmaduro}

\author{
AUTHORS: García R, Carrasco A, Bruno F, Estévez \\ $\mathbf{R}$, Cisneros R.
}

\section{Introduction}

The term "regenerative endodontics" defines those biologically designed procedures to replace damaged structures, including dentin and root structures, as well as cells of complex dentine-pulp, based on the three basic parameters derived from tissue engineering: stem cells, growth factors and matrix scaffold. It is a broader respect to the term "revascularization" which defines the restoration of blood flow to achieve the formation of the pulp tissue concept.

\section{Case Report}

To explain the various protocols in the literature, we present a case of a patient of 8 years old when he decided to perform the regeneration treatment pulp in the right central incisor with immature apex, diagnosed with pulp necrosis and symptomatic apical periodontitis . Among the main highlights solutions sodium hypochlorite at various concentrations, the high antimicrobial capacity, chosen in this case at a concentration of $2.5 \%$ solution. As for the medication, the most used is the triantibiotic paste combination of metronidazole, minocycline and ciprofloxacin. The main drawback is that produces staining on the tooth. For this reason, it was decided to place the combination of biantibiotic paste without minocycline.

Regarding the intracanal matrix, most authors decide simply induce clot. In this case, it was decided to place a collagen sponge with a double function: to provide a matrix scaffolding and facilitate compaction MTA.

\section{Conclusions}

It is important to note that there is no single established protocol, and major differences appear in the choice of irrigant and medication, the possible placement of a matrix as a scaffold, and the need for mechanical cleaning of previous root canal disinfection.

\section{- Oral Presentation 65 \\ TITLE: Eficacia de las técnicas de regeneración pulpar: caso clínico}

\section{AUTHORS: Santana Hernández G, Ónega Seoane R, León Nuñez I, Bruno F, Cisneros Cabello $R$.}

\section{Introduction}

The treatment of an immature tooth with pulp necrosis and apical periodontitis is today one of the major challenges in the world of endodontics. The thin and fragile walls of the root canal system that characterize these teeth difficult to carry out a proper mechanical preparation and a thorough chemical cleaning, as well as the possibility to obtain a good apical seal in the sealing process.

When we find this traditional treatment clinical situation relates to the apexification, which aims to promote the formation of an apical barrier and achieve closure of the apex of a non- vital immature tooth. The main problem with this treatment is based on the inability to achieve a normal root development, with thickening of dentinal walls and root elongation.

Studies of the last 50 years have focused on the technique of revascularization, based on the restoration of blood flow in the empty root canal of an immature tooth, hoping to trigger a process similar to that obtained healing after conventional surgical wound but we face a changing concept, strictly linked to the principles of regeneration, advances in terms of disinfection and using new materials.

\section{Case Report}

Here are a number of clinical cases in which one can see the great result of treatment, based on the resolution of apical periodontitis and the absence of clinical signs and symptoms, as well as an increase in the length and width of the root. In the first session we proceeded to disinfect the root complex by antibacterial irrigating solutions (sodium hypochlorite), followed by placement of a medicament for enhancing the antibacterial action, being the triantibiotic paste and calcium hydroxide most employees. In the second session we proceeded to induction of blood clot manually, be possible to add a scaffold matrix and then a plug of MTA. We monitor both clinical (absence of symptoms) and radiographic (closure of apical foramen, elongation and thickening of the root walls). 


\section{Conclusions}

There is no specific or standardized protocol.

The differences appear in materials used, mechanical and chemical cleaning and use of matrix as a scaffold.

\section{- Oral Presentation 66}

TITLE: Pulp revascularization of a necrotic permanent incisor using plasma rich in growth factors

\author{
AUTHORS: Yanes Barroso E, Gaviño Orduña JF, \\ Barrientos Delgado JF, López López J, Segura-Egea \\ JJ.
}

\section{Introduction}

The pulp revascularization is a promising alternative for the treatment of immature necrotic teeth with apical periodontitis. The scientific literature provides abundant evidence of success in patients 8-16 years, but is low in relation to results in adult patients. The following case reports the results of revascularization of necrotic incisor in an adult patient in which autologous plasma rich in growth factors (PRGF) was used as adjuvant. Follow up the case with radiographs and CBCT was performed for 5 years.

\section{Case Report}

A woman of 35 years requires dental care due to pain and discomfort in the left maxillary central incisor, affected by trauma when he was 8 years old. At the intraoral examination discoloration and a sinus tract was observed in buccal mucosa. Percussion and vitality tests were negative. In the periapical radiograph an incomplete root development and a radiolucent periapical lesion was observed. Based on the clinical and radiological findings pulp necrosis was diagnosed with chronic apical periodontitis. The treatment performed was pulp revascularization using PRGF as scaffold and to supply growth and survival factors. The clinical and radiological follow-up showed, after 5 years, a decrease in radiolucent lesion size and thickening of the root walls with the presence of a calcified structure forming bridges occupying the root canal.

\section{Conclusions}

Although it is only a case report, their results suggest that revascularization treatment with PRGF in an open apex tooth with and apical periodontitis also appears to be feasible in adults.

\section{- Oral Presentation 67}

TITLE: Non-surgical endodontic retreatment and $y$ restoration of 1.1 with large apical diameter
AUTHORS: Sanchez Orti A, Ceballos Velo E, Baena

E.

\section{Introduction}

A non-surgical endodontic retreatment implicates the re-entrance to the radicular canal system in order to remove the previous obturation material and allow a proper chemomechanical debridement. Specific strategies in terms of irrigation, instrumentation and sealing material which guarantee a good apical seal must be taken into account when the apical diameter is large. Furthermore, an adequate posterior restoration is essential to achieve a coronal seal.

\section{Case Report}

An adult female was referred for endodontic retreatment in tooth 1.1 due to the need to replace crowns in 1.1 and 2.1. After exploration, an asymptomatic, infraobturated and poor condensed endodontic treatment was detected. After coronal access was carried out with the aid of magnification, preflaring was performed (SX, ProTaper Universal, Dentsply Maillefer) and gutta-percha was removed with 10 and $15 \mathrm{~K}$-files (Dentsply Maillefer). Patency and WL was achieved (LEA, Root ZX Mini, Morita) and the canal was instrumented with second set files. Apical diameter was established at \#80. EndoVac (Kerr) was the irrigation system of choice to avoid the extrusion of irrigant $(5.25 \% \mathrm{NaOCl})$. After final irrigation protocol, the canal was dried and a $4 \mathrm{~mm}$ apical MTA plug (ProRoot, Dentsply Maillefer) was done. One week later, post space was prepared and the fiber post (1.4 mm diameter, GC Fiber Post, GC) was cemented with RelyX Unicem (3M ESPE). The cavity access was restored with composite (A2 G-aenial Universal Flo, GC) after applying a total-etch adhesive technique (Adper Scotchbond 1XT, 3M ESPE). Finally, temporary crowns (Structure 3, VOCO) were cemented.

\section{Conclusions}

The non-surgical retreatment on crowned cores which present a large apical diameter implies a specific strategy along the treatment to ensure a successful outcome. The posterior restoration with fiber post prevents the fracture of the core which will receive lateral and shearing forces during mastication.

\section{- Oral Presentation 68 \\ TITLE: Multidisciplinary treatment of a frac- tured teeth with open apex: apex seal with MTA}

AUTHORS: Torres-González I, Conde-Pais J, Costas-Soto A, González-Villafranca P, Pozo-Fernández A, Otero-Ávila A. 


\section{Introduction}

Traumatic injuries in immature teeth can produce a pulp necrosis leading to the arrest of its development, and consequently, their root canals may present parallel or divergent walls in the apical third. This means an endodontic and restorative challenge, due to the difficulty to ensure cleaning and shaping of the canal and, moreover, prevents from accurate sealing.

Apexification is the most reccommended therapy to create an apical barrier wich provides a permanent and hermetic filling of the root canal. Mineral trioxide aggregate (MTA) is the material of choice to use with this technique due to its manipulation, biocompatibility and bioactive properties that success in achieving a proper seal for further root canal treatment.

\section{Case Report}

Proper patient who came to the Máster en Odontología Multidisciplinar Estética, University of Granada, referring tooth pain in the upper right central incisor (1.1) and fracture, without pulp affectation, due to dental trauma in his childhood. After testing vitality, percussion and complete radiographic examination, the diagnosis of pulp necrosis with chronic periapical periodontitis associated with incomplete development of the apical portion was established.

This situation required a multidisciplinary approach which included the apex seal with MTA, endodontic treatment of the canal, external and internal whitening and reconstruction was addressed by adhesive techniques with direct composite veneers

\section{Conclusions}

The disappearance of symptoms and the significant healing showed by control radiographs demonstrate success in achieving an apical barrier and subsequent root canal treatment.

The MTA is revealed as an excellent material of choice for situations where it is intended to induce apexification in teeth with underdeveloped apex. Furthermore, this technique reduces the processing time required for a classic apexification with calcium hydroxide.

\section{DIAGNOSIS-PATHOLOGY}

\section{- Oral Presentation 69}

TITLE: Prevalence of molar incisor hypomineralization in a group of southern Spanish children

\section{AUTHORS: Jiménez Moreno E, Cwierz López IM, Fernández Vargas AM, Segura Egea JJ.}

\section{Objectives}

To find the prevalence of molar incisor hypomineralization in a group of 6- and 7-year-old Southern Spanish children from the municipalities of Lebrija and El Cuer-vo.

To investigate the influence of gender and socioeconomic factors on molar incisor hypomineralization in this group.

\section{Materials and Methods}

The cross-sectional study that was carried out to determine molar incisor hypomineralization prevalence involved 423 children aged 6 and 7 year old from the 1st grade of compulsory school in the municipalities of Lebrija and El Cuervo, Seville province, Andalusia. Gender and location (rural and peri-uban areas) influence were studied. Also, socioeconomic status (according to the declared profession of the head of household) and nationality factors were examined from a voluntary data questionnaire filled by the parents.

Statistical analysis was performed using the chi-square test and ANOVA. A probability value of $p<0.05$ was regarded as statistically significant. Odds ratios with 95\% CIs were calculated to quantify the associations by univariate logistic regression.

\section{Results}

The prevalence of molar incisor hypomineralization was $39,61 \%$. Location was the only factor that showed statistically significant difference, being higher in peri-urban areas (OR: 2,05; 95\% CI $(1,098-3,835(p=0,02)$.

\section{Conclusions}

Prevalence of molar incisor hypomineralization was $39,61 \%$, excluding children that did not have any permanent molar.

Molar incisor hypomineralization occurence was higher in peri-urban zones (Lebrija) than in rural zones (El Cuervo). Gender, socioeconomic status and nationality were found not to be statistically significant.

\section{- Oral Presentation 70 TITLE: Caries in Mount Classification: clinical and radiological concordance}

AUTHORS: Hernando B, Aliaga I, Fernández C, García E, Gómez A, Vera V. 


\section{Objectives}

Evaluate the correlation between the visual recording of the caries lesion according to the Mount classification, with ortopantomography and intraoral radiology.

Relate the stage of the caries lesion of Mount classification, with the degree of radiological involvement of dental caries.

\section{Materials and Methods}

A cross-sectional study was designed, selecting 94 patients with suspected caries, between 2014 and 2015. All patients included were performed by a single observer, a clinical examination of the presence of caries, recording the findings according to the Mount caries classification. Two observers independently and masked, analyzed orthopantomography and bitewing radiographs and/or periapicals. The association was evaluated with the McNemar test and validity indices. Statistical analysis was performed with STATA, 12.0.

\section{Results}

94 patients were studied and 498 caries were evaluated by clinical examination, orthopantomography and intraoral $x$-ray. $83,3 \%$ of caries site 1 and $93,5 \%$ of site 3 , stage 0 or 1 , were recorded only visually. Of the 253 interproximal caries recordered $(50,8 \%), 59(23,32 \%)$ were only diagnosed through x-ray and related to grade 2 radiologically. Stage 3 and 4 related to grades 3 and 4 $\mathrm{X}$-ray respectively.

\section{Conclusions}

Mount classification properly recorded the dynamic nature of caries in the initial stages of the lesion. However, the most advanced stage suffer from the information provided by x-rays.

\section{- Oral Presentation 71}

TITLE: Diagnostic capability of laser-induced fluorescence-405nm in pulp exposure: a clinical study

\section{AUTHORS: Bonilla-Represa V, Herrera-Martínez M, Rodríguez-Vázquez MM, Ábalos-Labruzzi C.}

\section{Objectives}

To clinically determine whether laser-induced fluorescence (LIF) with $\lambda=405 \mathrm{~nm}$ was able to assess pulp tissue health or disease in situations of pulp exposure.

\section{Materials and Methods}

68 healthy subjects were scheduled for the treatment of pulp. Three groups were established according to pulp tissue condition: Group A ( $n=30$ teeth) (pulp necrosis); Group B ( $n=30$ teeth) (irreversible symptomatic acute pulpitis) and Group $\mathrm{C}(\mathrm{n}=8$ teeth with accidental pulp exposure). We established two study groups for this purpose: a pulp necrosis group, $n=38(\mathrm{~A}+\mathrm{C}=$ eight healthy pulps and 30 necrotic pulps) and a pulpitis group, $n=$
$38(\mathrm{~B}+\mathrm{C}=$ eight healthy pulps and 30 cases of acute pulpitis). The carious lesions were eliminated, and LIF qualitative $(\lambda=405 \mathrm{~nm})$ measurements were made at one level: direct pulp exposure measurement (A-LP). The diagnostic performance of two systems LIF: SIROInspect (Sirona Dental Systems, Bensheim, Germany) and VistaProof (Dürr Dental GmbH \& Co.KG, BietigheimBissingen, Germany) in application to pulp tissue health or disease was assessed by calculating the sensitivity and specificity of the two tests at level A-LP.

\section{Results}

In relation to qualitative LIF $(\lambda=405 \mathrm{~nm})$, the necrosis group showed a sensitivity (S) of 0.7 and a specificity $(\mathrm{Sp})$ of $1.0(\mathrm{~S}+\mathrm{Sp}=1.7)$, while in the case of the pulpitis group $\mathrm{S}=0.88$ and $\mathrm{Sp}=1(\mathrm{~S}+\mathrm{Sp}=1.88)$.

\section{Conclusions}

This clinical study showed that qualitative laser-induced fluorescence to be useful in assessing pulp tissue health or disease. The technique may be useful to determine the prognosis and treatment of pulp tissue lesions. 


\section{ADHESION}

\section{- Oral Presentation 72 \\ TITLE: Adaptation of the sealant in Adaptación del sellador fissures with differents conditioning systems}

\section{AUTHORS: Melo M, Sittoni-Pino F, Ata-Ali J.}

\section{Objectives}

To determine which of the following treatments: etching with Ortophosphoric acid, Microabrasion, or both combined, favors a better adaptation of the sealant.

\section{Materials and Methods}

The sample ( $\mathrm{n}=66$ molars) was divided into 3 groups receiving different conditioning systems: etched with 37\% Ortophosphoric acid, Microabrasion (Aluminum oxide, 27 microns, 10 seconds, system Air-Flow Prep K1 Max, EMS) or both combined. Subsequently, pits and fissures sealant material was conventionally applied. Samples were stained with methylene blue and half of each group was thermocycled to be compared with the group without thermocycling (control group). Then, the teeth were cut with a diamond blade at low speed in buccolingual direction, obtaining cuts $1 \mathrm{~mm}$ thick, which were observed with an optical microscope (Nikon C-W10xB / 22) (X10). Dye penetration was assessed as proposed by Överbö and Raada scale $(0=$ no dye penetration, $1=$ penetration to half the sealant, $2=$ dye exceeds half the sealant). $p<0,05$ was considered statistically significant, with a confidence interval of $95 \%$.

\section{Results}

In all groups, microleakage was greater in those teeth that underwent thermocycling against the values of the control group $(p<0,05)$. The values of the groups that underwent thermocycling were $1.45,1.30$ and 0.73 for groups of acid etching, micro-abrasion and combined, respectively. When analyzing the differences between acid etching and combined techniques, we note that the differences are statistically significant $(p<0,05)$.

\section{Conclusions}

Microabrasion of the occlusal surface and subsequent acid etching (combined technique), gets the best results when compared with the use of acid etching and air abrasion in isolation.

\section{- Oral Presentation 73}

TITLE: Importance of the light curing mode in the adhesión of bulk-fill resins

AUTHORS: Funes Gil I, Báguena Gómez JC, Chiva García F.

\section{Objectives}

To evaluate the influence of light curing mode in the microtensile bond strength to dentin of two bulk-fill resin composite using universal adhesives.

\section{Materials and Methods}

An occlusal quadrangular cavity of $4 \mathrm{~mm}$ in depth with $3 \mathrm{~mm}$ of surrounding enamel was prepared in 42 sound human molars. They were randomly assigned for subsequent filling to 4 groups: G1: X-tra fil ${ }^{\circledR}+$ Futurabond $\mathrm{U} 囚($ Voco) light cured in continuous mode $(\mathrm{n}=10), \mathrm{G} 2$ : X-tra fil ${ }^{\circledR}+$ Futurabond $U \circledR$ light cured in pulsed mode $(\mathrm{n}=10), \mathrm{G} 3$ : QuixFil ${ }^{\circledR}+$ XenoSelect ${ }^{\circledR}$ (Dentsply) in continuous mode $(n=11)$ y G4: QuixFil ${ }+$ XenoSelect ${ }^{\circledR}$ in pulsed mode $(\mathrm{n}=11)$. Adhesives were used in self-etching mode and light cure to $4 \mathrm{~mm}$ distance. In all specimens was used a mini LED ${ }^{\circledR}$ lamp (Satelec). After removing the surrounding enamel, the teeth were stored 24 hours at $37^{\circ} \mathrm{C}$. Then they were sectioned to obtain axially prismatic specimens of $\sim 10 \mathrm{~mm}^{2}$ length and $\sim 1 \mathrm{~mm}^{2}$ section, and 40 were randomly selected for each group $(n=160)$. They were subjected to microtensile bond strength in a universal testing machine Autograph AGS-1 KND whose head speed was $1 \mathrm{~mm} / \mathrm{min}$. Those values were statistically analyzed by analysis of its variance (ANOVA) and Tukey's test for multiple comparisons post.

\section{Results}

There were no significant differences between the two modes of light curing comparing each complete system universal adhesive + composite resin separately $(p>0,05)$. However, when compared with each other statistically significant differences were observed $(p=0,001)$ between X-tra fil ${ }^{\circledR}$ and QuixFil ${ }^{\circledR}$ in pulsed mode $(36.10 \pm 30.10$ $\mathrm{MPa}$ vs $19.36 \pm 33.76 \mathrm{MPa}$ ) as well as in continuous mode (43.28 $\pm 29.68 \mathrm{MPa}$ vs. $14.39 \pm 25.47 \mathrm{MPa}$ ).

\section{Conclusions}

Light curing mode does not influence microtensile bond strength. The complete system XenoSelect ${ }^{\circledR}$ with QuixFil ${ }^{\circledR}$ showed less bond strength to dentin in self-etching mode than FuturabondU ${ }^{\circledR}$ with X-tra fil ${ }^{\circledR}$ system.

\section{- Oral Presentation 74 TITLE: Use of Universal adhesives as primers in porcelain and zirconia adhesion}

\author{
AUTHORS: Sánchez Alcaraz E, Funes Gil I, Chiva \\ García F.
}

\section{Objectives}

To review the scientific literature discussing the efficacy of universal adhesives as primers in porcelain and zirconia adhesion.

\section{Materials and Methods}

A systematic review was performed in MEDLINE (PubMed) database. The following MeSH terms, search 
terms were used: "universal adhesive", "silane", "porcelain", "zirconia" and "10-MDP monomer". As inclusion criteria, the articles should contain information about universal adhesives, their use in porcelain and zirconia and/or adhesion factors and mechanisms in porcelain and zirconia with a publication date of 10 years (20062016). Language was restricted to English. From a total of 92 items found, 29 were employed.

\section{Results}

Universal adhesives can be used on different substrates, both dental and non-dental using both methods of adhesion (etch and rinse and self-etching) using a single product.

Not all the articles support the clinical efficacy of universal adhesive as primer in porcelain and zirconia as it presents multiple problems arising from the interaction of various of its components, such as 10-MDP and Bis GMA in the same bottle with silane, resulting in a loss of its properties. Good adhesion results to silicabased ceramics have been observed, but not to zirconia because of its low silica content (1\%). In this case, the presence of the monomer 10-MDP in the adhesive composition for a chemical bond to zirconia is necessary. Universal adhesive with 10-MDP monomer require no pretreatment of the bonding surfaces and its bonding strength decreases with time due to hydrolysis of the adhesive components when the silane is added.

\section{Conclusions}

Universal adhesives have been introduced into the market to simplify bonding procedures but they are not recommended as primer in zirconia and porcelain due to the interaction of its components.

More scientific evidence to support their use in restorations of porcelain or zirconium is necessary.

\section{- Oral Presentation 75}

TITLE: Bonding strength of self-adhesive resin cements used on feldspathic ceramic with preetched enamel surface

\section{AUTHORS: Geng Vivanco R, Saravia Rojas MA.}

\section{Objectives}

Compare the bonding strength of three self-adhesive resin cements used on feldspathic ceramic, being the enamel surface pre-etched for 15 seconds with $37 \%$ phosphoric acid.

\section{Materials and Methods}

Feldspathic ceramic Vitadur alpha VM7 (Vita, Germany) were used. N: 147 specimens. GI (Control) $n=35$ : $10 \%$ hydrofluoric acid Condac Porcelain (FGM, Brazil) for 1 minute + Silane Prosil (FGM, Brazil) + RelyX ARC (3M ESPE, USA); GII $n=38: 10 \%$ hydrofluoric acid Condac Porcelain (FGM, Brazil) for 1 minute +
Silane Prosil (FGM, Brazil) + RelyX U200 (3M ESPE, USA); GIII $n=34: 10 \%$ hydrofluoric acid Condac Porcelain (FGM, Brazil) for 1 minute + Silane Prosil (FGM, Brazil) + Permacem 2.0 (DMG, Germany); GIV n=40: $10 \%$ hydrofluoric acid Condac Porcelain (FGM, Brazil) for 1 minute + Silane Prosil (FGM, Brazil) + BisCem (Bisco, USA); cemented to etched enamel. The samples were stored in saline solution for 24 hours and then they were sectioned with a cutting machine to obtain specimens of $1 \times 1 \times 6 \mathrm{~mm}$. The specimens were stored in saline at $37^{\circ} \mathrm{C}$ for 24 hours before micro-tensile bond strength testing was carried out with the microtensile tester (Bisco, USA) at a crosshead speed of $0.5 \mathrm{~mm} / \mathrm{min}$. The data were analyzed with ANOVA and Tukey's test.

\section{Results}

The self-adhesive resin cements RelyX U200, 3M; Permacem 2.0, DMG y BisCem, Bisco had higher bond strength $(13.20 \pm 3.47 \mathrm{MPa}, 13.67 \pm 5.11 \mathrm{MPa}, 10.79 \pm 4.86$ $\mathrm{MPa}$; respectively) than the cement RelyX ARC, 3M $(7.86 \pm 3.37 \mathrm{MPa})$, statistically significant differences were found $(p<0.05)$. Permacem 2.0 showed significantly higher bond strength in comparison to Biscem $(p<0.05)$.

\section{Conclusions}

The cement Permacem 2.0 revealed the highest bond strength of the cements used in the present study.

\section{- Oral Presentation 76 \\ TITLE: Universal adhesives. A literatura review}

\section{AUTHORS: Irazusta S, Ramirez A, Valles M.}

\section{Objectives}

A literature review to meet long-term and clinical behavior in vivo of universal adhesives stability studies were performed.

\section{Materials and Methods}

Original scientific papers of universal adhesives in vivo studies published in the MEDLINE (PubMed) database since febreary of 2016 were included in this systematic review. Inclusion criteria was in vivo studies with a minimum follow-up period of 6 months and one of the groups using universal adhesives. A total of 6 articles were selected from the 16 articles initially identified.

\section{Results}

We don't found statistically differences in clinical behavior, but reporting differences when it is applied without prior acid etching.

The advantages of universal adhesives are its simplicity, its adhesion to surfaces of enamel, dentin and various types of restorative materials, the possibility of using the technique of etch and rinse or self-etch, with good adhesion values and marginal sealed and marked reduction of postoperative sensitivity. 


\section{Conclusions}

Based on the results of this systematic review in a period up to 48 months, universal adhesives offer a good clinical stability.

\section{- Oral Presentation 77}

TITLE: Effect of Hydroxyapatite in self-etching and etch-and-rinse adhesives: mechanical strenght and sealing

\section{AUTHORS: Pozo Fernández A, Del Castillo Salme- rón R, Rosales Leal JI.}

\section{Objectives}

To evaluate the effect of Hydroxyapatite on the mechanical strenght and sealing of self-etching and etch-andrinse adhesives.

\section{Materials and Methods}

24 third molars, extracted in the previous six months and thymol preserved, were sectioned and Xeno Select ${ }^{\circledR}$ (Dentsply, Konstanz, Germany) adhesive was applied on the dentin surface, categorizing these molars into 4 classes: Xeno ${ }^{\circledR}-A$, etch-and-rinse plus Xeno ${ }^{\circledR}-B$, Xeno ${ }^{\circledR}$ with a hydroxyapatite-C filling, and etch-andrinse plus $X e n o{ }^{\circledR}$ with a hydroxyapatite-D filling. 12 samples were connected to a pulp pressure device for 24 hours in a stove at $37^{\circ}$ degrees and their axials were observed under CLSM with two fluorochromes ( rhodamine and fluorescein) to evaluate the sealing and the bonding interface's micropermeability. The other 12 samples underwent a micro tensile test by compression to analyze the mechanical strenght. The multivariate analysis of variance ANOVA and the T-student test $(p<0,05)$ were used in the statistical analysis.

\section{Results}

The hybrid layer and the thickest adhesive layer were found in the total-etch group with a Hidroxiapatita (D) filling. Among the total-etch groups a higher number of tubules opened by micra were found, as well as a higher number of sealed tubules and a greater sealing lenght.

The groups that obtained the highest resistance to microtension were the total-etch ones.

\section{Conclusions}

The hydroxyapatite filling does not increase microtensile strenght but it does increase the types of cohesive failures on the substratum and the number of sealed tubules. The etch-and-rinse produces a higher interaction with the substratum increasing the number of exposed tubules and permeability.

\section{- Oral Presentation 78 \\ TITLE: Adhesion: conventional glass ionomers vs resin modified glass ionomers}

\author{
AUTHORS: Seguí Troth A, Castillo Felipe C, Chiva \\ García F.
}

\section{Objectives}

To review the literature and determine if bonding properties of conventional glass ionomers are modified in resin modified glass ionomers.

\section{Materials and Methods}

An electronic research which included articles published in the last 5 years were made using Medline database. In vitro and systematic review articles were included. Keywords: glass ionomer, resin-modified glass ionomer, bonding, dentin.

\section{Results}

lass ionomer has the ability to quemically bond to structures with polarity such as dentin, enamel and bone. Free carboxylic groups form hydrogen bonds with the substrate, when the reaction matures, hydrogen bonds transform into stronger ionic bonds. Resin modified glass ionomers result from incorporating methacrilate acid to poliacrilic acid. Resin tags which are formed increase contact surface, dentine humectability and a closer relationship between dentine and glass ionomer which contributes to mechanically retain and quemically bond. Setting is firstly accomplished by photoactivation followed by acid-based reaction when water is absorbed.

Authors found that bonding strength values of resinmodified glass ionomers were higher after 24 hours, and some in vitro studies showed that after 6 months bonding values were similar in both materials. Previous conditioning of RSMGI and CGI with poliacrilic acid showed higher bonding values. No clinical studies were found.

\section{Conclusions}

Previous dental conditioning improves dental bonding strength, long term bonding properties are similar and more studies are necessary.

\section{- Oral Presentation 79}

TITLE: Influence of the acid conditioning in enamel on adhesion with self-adhesive cements

\author{
AUTHORS: Saravia Rojas MA, Nima G, Abuna G, \\ Tay Y, Puppin-Rontani RM.
}

\section{Objectives}

Evaluate the influence of different conditioning times (37\% acid phosphoric for 5", 10" and 20") in the enamel 
and the effect on the bond strength (BS) with self-adhesive cements (SC) subjected to thermocycling (TC).

\section{Materials and Methods}

128 blocks of bovine enamel of $8 \times 4 \times 3 \mathrm{~mm}$ were randomly divided in 2 cements, RelyXU200 (XU), MaxCem Elite (MC). 4 subgroups $(\mathrm{n}=16)$ were formed for each cement, according to the conditioning time. G1: Control, G2: 5 seconds, G3: 10 seconds, G4: 20 seconds. Three SC cylinders $(1 \mathrm{x} 1 \mathrm{~mm})$ were built on each block. The specimens were stored in distilled water at $37^{\circ} \mathrm{C}$ for 24 hours. After that, half of them were subjected to a microshear test in a universal testing machine, applying a crosshead speed of $0.5 \mathrm{~mm} / \mathrm{min}$, to produce the fracture. The other half was subjected to 5000 thermal cycles $\left(5^{\circ} \mathrm{C}-55^{\circ} \mathrm{C}\right)$ before the test. The type of failure was analyzed with a scanning electron microscope (SEM). The results (MPa) were analyzed using the three-way ANOVA test and the intergroup differences with the Student's t-Test or Mann-Whitney U test $(p<0,05)$. The interface layer was analyzed with confocal laser scanning microscopy and SEM.

\section{Results}

Conditioning significantly improves the BS when compared with control groups $p<0,05$. The TC negatively affect the $\mathrm{BS}$ of the groups without conditioning $p<0,05$. Among the groups with the conditioning, the BS didn't show difference between without TC and with TC, $p>0,05$. Predominantly adhesive and mixed fractures in the groups without conditioning and conditioning were observed, respectively. There was no resin tags in the groups without conditioning.

\section{Conclusions}

Conditioning on the enamel must precede the use of (XU) and (MC). Conditioning 5" (XU) and 10" (MC) generated appropriate BS.

\section{- Oral Presentation 80}

TITLE: Treatment of dentinal hypersensitivity by using dentinal adhesives

\section{AUTHORS: Chiva García F, Fernández Márquez A, Ferrández Pujante A.}

\section{Objectives}

To update the knowledge on the treatment of dentinal hypersensitivity by using dentinal adhesives.

\section{Materials and Methods}

A literature review of the last 16 years (2000-2015) was performed in the Medline database. Keywords: "dentinal hypersensitivity", “ cervical dentinal sensiti-vity", "dental pain", "bonding system", "desensitizing agents".

\section{Results}

Numerous studies conclude that the dentinal adhesives are capable of sealing the dentinal tubules and reduce permeability, significantly decreasing the sensitivity immediately in cases of exposed cervical dentine and staying up to 3 months after treatment. The effectiveness of adhesives of one bottle to reduce dentinal hypersensitivity, with and without prior acid etching, was evaluated in different studies, with similar results absence of spontaneous sensitivity, maintained significantly at 24 weeks of treatment in both cases. Over time, the adhesive breaks away resulting in exposure of the tubules, so this technique is generally reserved for cases of specific and localized dentinal hypersensitivity rather than generalized dentinal pain.

\section{Conclusions}

The dentinal adhesives are valid alternative for the treatment of dentinal hypersensitivity immediately. In the long term, its effectiveness is affected by the passage of time.

\section{- Oral Presentation 81 \\ TITLE: Influence of surface treatment in lithi- um dissilicate bond strength}

\author{
AUTHORS: Pérez Sánchez LE, Cura M, Fuentes V, \\ Ceballos L.
}

\section{Objectives}

To determine the bond strength of a resin cement to a lithium dissilicate ceramic after surface treatment with several materials: Monobond Plus, Scotchbond Universal and the new Monobond Etch\&Prime.

\section{Materials and Methods}

20 samples of lithium dissilicate ceramics, IPS e.maxCAD (Ivoclar-Vivadent) of 10x10x1mm dimensions were randomly assigned to five groups: 1 . Monobond Plus (Ivoclar-Vivadent) 2. Scotchbond Universal (3M ESPE) 3. Monobond Plus + Scotchbond Universal 4. Monobond Etch\&Prime (Ivoclar-Vivadent) 5. Monobond Etch\&Prime + Scotchbond Universal. For groups 1,2 and 3 the ceramic was previously etched with $4,9 \%$ hydrofluoric acid for 20 seconds (Ceramic Etching Gel, Ivoclar-Vivadent) and for groups 4 and 5 Monobond Etch\&Prime was directly applied according to the manufacturer's intructions. Afterwards the above mentioned treatments, $0,8 \mathrm{~mm}$ diameter cylinders of Variolink Esthetic DC (Ivoclar-Vivadent) were luted. After photopolymerization, samples were submitted to a microshear test in a universal testing machine (Instron 3345). Data were analyzed by ANOVA and Tukey tests $(p<0.05)$. The type of failure was determined and selected specimens were observed under a scanning electron microscope.

\section{Results}

Group 3, Monobond Plus + Scotchbond Universal yielded the highest bond strength values, without significant differences with groups 4 and 5 . 


\section{Conclusions}

Monobond Etch\&Prime either with or without the subsequent use of a Universal adhesive obtained similar bond strength results to the conventional treatment with Monobond Plus + Scotchbond Universal, after hydrofluoric acid etching.

\section{- Oral Presentation 82}

TITLE: Influence of proximal box elevation technique and resin cement used on marginal sealing of composite inlays

\section{AUTHORS: Da Silva D, Fuentes MV, Ceballos L.}

\section{Objectives}

To evaluate the influence of proximal margin elevation technique on marginal sealing of composite inlays, using a conventional "total-etch" resin cement and a selfadhesive one.

\section{Materials and Methods}

MOD inlay preparations were performed in 18 human third molars. According to proximal margin position, three situations were defined: margin in enamel $(1 \mathrm{~mm}$ above cementoenamel junction), in dentin (1 $\mathrm{mm}$ below cementoenamel junction) or elevated with resin composite to $1 \mathrm{~mm}$ above cementoenamel junction (Adper Scotchbond 1XT and Filtek Z250, 3M ESPE). Inlays were fabricated with Gradia Indirect (GC) and luted with a "total-etch" conventional resin cement, RelyX ARC (3M ESPE), or with the self-adhesive resin cement G-Cem (GC), following manufacturers' instructions. After one week water storage $\left(37^{\circ} \mathrm{C}\right)$, marginal sealing was determined by nanoleakage test. Results were analyzed by Kruskal-Wallis and Mann-Whitney U tests $(p<0.05)$.

\section{Results}

Statistical significant differences in marginal sealing capacity were detected among experimental groups. The lowest values of nanoleakage corresponded to the interface established between resin composite used for marginal elevation and composite inlays, and they were statistically similar to the obtained for composite inlays with proximal margins in enamel, regardless of the resin cement used. Higher nanoleakage was significantly determined in the interface established between dentin and the resin composite used for marginal elevation.

\section{Conclusions}

Higher nanoleakage values were obtained for the interface established between dentin and the resin composite used for marginal elevation applying a "total-etch" adhesive system in comparison with interfaces with margins in enamel and dentin.

\section{- Oral Presentation 83 \\ TITLE: Estado actual de la citotoxicidad de los adhesivos dentinarios}

\author{
AUTHORS: Mercado Díaz AM, García Belando A, \\ Chiva García F.
}

\section{Objectives}

to determine the toxicity of dental adhesives on oral tissues.

\section{Materials and Methods}

A literature search in Medline and TESEO data was performed.

The search keywords were: dental monomers toxicity, toxicity dental adhesives, restorative dentistry toxicity, filling toxicity. Fifteen items according to the inclusion criteria were used. The inclusion criteria were: i) reference to the cytotoxicity of the adhesive on the oral tissues, ii) no more than five years old.

\section{Results}

it has demonstrated the cytotoxic effect of dental adhesives on fibroblasts and pulp cells, which is dose dependent. BisGMA based adhesives are more cytotoxic than those containing UDMA, TEGDMA and HEMA (BisGMA $>$ UDMA $>$ TEGDMA $>$ HEMA). However, the interaction of two or more components may decrease or increase the cytotoxicity of the adhesive. Similarly, other important components such as dimethoxy (DMBZ) and tetradecyldimethylamine (DMDTA) have severe cytotoxic effects while dimethyl-p-toluidine (DMPT), benzylmethacrylate (BEMA) and camphorquinone (CQ) show a mild cytotoxicity. Another aspect is the dentine thickness, which influences the concentration and amount of adhesive agents that penetrate through the dentin in the pulp space. Moreover, the two-step etching adhesives based HEMA, regardless of concentration diffusion, show a lower toxicity than those of a single step.

\section{Conclusions}

there is a widespread consensus on the cytotoxic potential of dental adhesive systems in animal and human cells evaluated. The cytotoxicity of binding agents evaluated are mainly due to the monomers present in the composition and, particularly, residual unpolymerized monomers. Finally, the two-step self-etching adhesives are more biocompatible than those of a single step. 


\section{INLAY-OVERLAY}

\section{- Oral Presentation 84 \\ TITLE: Marginal adaptation of composite over- lays with conventional impression and digital scanner}

\author{
AUTHORS: Garcia Cuesta C, Botello Torres R, Faus \\ Matoses V, Faus Matoses I, Faus Llácer V.
}

\section{Objectives}

Evaluation of marginal adaptation of composite overlays with different impression technique one with conventional silicone (Express ${ }^{\mathrm{TM}}$ VPS Impression Material, 3M ESPE, Seefeld, Germany) against a digital impression with the scanner True Definition (3M ESPE, Seefeld, Germany).

\section{Materials and Methods}

70 extracted third molars were divided into two groups $(n=35)$. MOD cavities were prepared to obtain composite overlays. In the first group silicone was used to take the impressions, after that the laboratory technician made the restorations; against the other group where an intraoral scanner took the impressions and the restorations were made by CAD/CAM. All the overlays were seen under the binocular stereoscopy magnifying glass MZ APO with 32x of magnification; images of the vestibular, lingual, mesial and distal surfaces were captured and sent to a computer with a measure ruler in micrometre. First of all was evaluated the passive adjustment, consider as the adaptation of the overlay dropped in the model. The gaps have been measure in micrometre following a perpendicular line from the internal surface of the restoration to the external surface of the tooth. With the Power Point (Microsoft office) application the distance between tooth/restoration had been measured in micrometre. After cementation the marginal adaptation was evaluated too. The ANOVA analysis and the Bonferroni test were used to achieve the medias of the marginal adaptation, before and after cementation.

\section{Results}

The scanner group got better results than the silicone one. Nevertheless, significant difference was just achieved after cementation $(p=0,022)$. In both groups, the best results were obtained in the oclusal surface ( $p=0,016$ scanner; $p<0,001$ silicone); the gingival angle got the fewer adaptation in the two groups (scanner 184,69 $\mu \mathrm{m}$; silicone 242,29 $\mu \mathrm{m}$ ).

\section{Conclusions}

Digital scanners are an alternative with favourable results in the impression taking during the clinical practice.

\section{- Oral Presentation 85}

TITLE: Which material do you use when make an inlay?

\section{AUTHORS: Moradas Estrada M, Villa Vigil MA.}

\section{Objectives}

Determine what material offers better marginal adaptation. Establish that material meets optimal biomechanical properties and to what situation. Compare the aesthetic capacity of each garment material.

Materials and Methods

A selective search was conducted in PubMed articles using the keywords: inlay, onlay, overlay, ceramic indirect restorations y Gold cast inlays and onlays. A second filter limiting the search of 1200 references to 118 items with high impact factor published in the last 10 years, noting a total of 15 that met the inclusion criteria spent quantitative and qualitative, the proposed use of materials and techniques.

\section{Results}

It was seen as traditional alloys, high noble metal provide them with excellent physical properties regarding biomechanical strength, marginal adaptability and po-lishing. But the search for an adaptation below $120 \mathrm{um}$, has been and is a constant that was not achieved with the use of cementitious agents, even prior adhesive preparation in ceramic inlays, with respect to the integrity or marginal adaptation of inlays ceramic before and after cementation. This justifies that the best clinical survival data are currently using computer-aided material such as CAD - CAM.

\section{Conclusions}

With the limitations of this study and the need to deepen the long-term evidence, we determine that: inlays inlay / onlay and overlay type are the best therapeutic alternative for the rehabilitation of molars and premolars with extensive loss of dental tissue due to cavities and fractures.Inlaid with ceramic and noble materials are accepted techniques with predictable rates of long-term success. cupídeos coatings offer better biomechanical properties against occlusal forces. Gold is still the best material when we demand marginal adjustment.

\section{- Oral Presentation 86}

TITLE: Influence of post placement on the fracture resistance of premolars with overlay

AUTHORS: Teruel Rodríguez A, Alegre Domingo T, Faus Matoses V, Faus Matoses I, Faus Llácer VJ.

\section{Objectives}

The aim of this study was to investigate the influence of post placement on the fracture resistance of endodonti- 
cally treated maxillary premolars restored with direct overlay composite restorations.

\section{Materials and Methods}

This study included 40 human maxillary premolars extracted for periodontal or orthodontic reasons.

Standarized mesio-occluso-distal cavities were prepared, teeth were endodontically treated and an occlusal reduction of $2 \mathrm{~mm}$ was performed. The specimens were divided in two groups $(\mathrm{n}=20)$. Group 1: Restored with direct composite cuspal coverage; Group 2: Restored with a fiber post and direct composite cuspal coverage. Teeth were embedded in polymerized acrylic resin and submitted to a compressive force applied 30 degrees to the long axis of the tooth until fracture. The results were statistically analyzed by one-way ANOVA and Tukey post hoc test $(P<0.05)$.

\section{Results}

Statistical analysis revealed no significant difference $(p>0.05)$ between the main failure loads of maxillary premolars restored with or without post and cuspal coverage.

\section{Conclusions}

Within the limits of this laboratory investigation it is concluded treated maxillary premolars with severely reduced dental structure restored with or without post and cuspal coverage have similar fracture resistance, which suggest that posts are not necessarily required when cuspal coverage is performed.

\section{- Oral Presentation 87}

TITLE: Clinical evaluation of the type of impression on the marginal adaptation of ceramic inlays

\section{AUTHORS: Vargas-Corral FG, Vargas-Corral AE, Cabrerizo-Vilchez MA, Rosales-Leal JI.}

\section{Objectives}

The aim of this study is to compare the marginal fit of ceramic inlays made from an impression with conventional silicone, with the marginal fit of other ceramics made with a digital intraoral impression system.

\section{Materials and Methods}

20 participants with a prosthetic need were selected for this study. Ten inlays were designed and fabricated with an intraoral digital impression system (IDI Group). The other ten inlays were custom made with a conventional silicone impression method with a two-step technique, and the resulting model was scanned with the same digital impression system (EDI Group). All the inlays were milled in feldespathic ceramics. In order to replicate the marginal fit, each Inlay was placed on its corresponding clinical preparation without cement; once placed, samples where taken even with silicone fluid of polyvinyl siloxane (PVS) in the occlusal surfaces and in the free faces. Three scanning of each sample were performed by White Light Confocal Microscopy (WLCM) with a scanning area $694.41 \times 510.09 \mu \mathrm{m}^{2}$.From each scanning 3 measures were taken of the distance InlayClinic Preparation and the discrepancy in height InlayClinic Preparation interface. Data was analyzed using descriptive statistics and a analytical statistical T-test $(p<0.05)$.

\section{Results}

The values of marginal adaptation on occlusal surfaces were significantly affected by the impression method used $(p<0.001)$. The values of the discrepancy in height were $62 \pm 44 \mu \mathrm{m}$ in occlusal and $89 \pm 68 \mu \mathrm{m}$ in the free faces for IDI group and $107 \pm 61 \mu \mathrm{m}$ in occlusal faces and $94 \pm 62 \mu \mathrm{m}$ in free faces to EDI group $(p<0.05)$.

\section{Conclusions}

Ceramic inlays made with intraoral scanning technique showed better occlusal marginal fit than those made from a silicone impression. 


\section{COLOR}

\section{- Oral Presentation 88}

TITLE: Incidence and perception of dental and labial alterations in the aesthetics of the smile

\author{
AUTHORS: Fernández Márquez A, Nicolás Silvente \\ AI, Chiva García F.
}

\section{Objectives}

The aim of this study was to determine which parameters in the smile aesthetics were altered, which were perceived by participants and how they affected the self-satisfied smile.

\section{Materials and Methods}

29 dentistry students were recorded. 3 photographs were taken: smile, maximum intercuspation and rest, on each participant, color and length of upper central incisors were taken. A survey of personal data and the perception of different parameters of the smile were collected. Pictures were analyzed through a template. The obtained data were statistically evaluated with Fisher exact test and Mann-Whitney to compare the results.

\section{Results}

More than half of participants had altered the smile line $(58.6 \%)$ and tooth position $(55.2 \%)$, while the parameters that caused greater dissatisfaction were position $(55.2 \%)$ and color $(17.2 \%)$. When comparing satisfaction and real alterations only dental position presented statistically significant differences $(p=0.003)$. Color was the only variable predictive of satisfaction $(p=0.01)$.

\section{Conclusions}

Smile line and dental position were the most altered para-meters, dental position and color are the parameters which most affected the aesthetics of the complacency smile.

\section{- Oral Presentation 89}

TITLE: Effect of green tea in the colour stability of a flowable composite resin

\section{AUTHORS: Pavan Robles F, Nicolás Silvente AL, Chiva García F.}

\section{Objectives}

To assess the effect of green tea in the colour stability of a new high loading nano-hybrid flowable composite resin (Inspiro ${ }^{\circledR}$, Axis Dental, Switzerland)

\section{Materials and Methods}

15 disks were made $(7 \mathrm{~mm} \varnothing \times 3 \mathrm{~mm})$ with $2 \mathrm{~mm}$ of i4 composite dentin and $1 \mathrm{~mm}$ of Inspiro ${ }^{\circledR}$ Flowable, which were polished with rubber polishing cup with diamond particles (Axis Dental, Switzerland). 3 groups were established $(n=5)$ according to the flowable composite used: Group 1: i4+Flowl; Group 2: i4+FIow4 and Group 3: i4+Flow7. Samples were immersed for 5' every day in green tea $\left(65^{\circ} \pm 10^{\circ} \mathrm{C}\right)$ for 10 days and kept in physiological saline solution at $36^{\circ} \mathrm{C}$. Colour was measured by Vita EasyShade digital spectrophotometer at the beginning of the study and after 10 days. The colour was assessed according to the CIEL-ch space. Results were analysed by means of ANOVA and LSD post-hoc test.

\section{Results}

Reduction of value $\left(\mathrm{L}^{*}\right)$ and chroma $\left(\mathrm{c}^{*}\right)$ and increase of hue $\left(\mathrm{h}^{*}\right)$ was observed after immersion in green tea. There were no significant differences between the three Inspiro ${ }^{\circledR}$ flowable colours as regards brightness (L*) and hue $\left(\mathrm{h}^{*}\right)$, but there were regarding saturation $\left(\mathrm{c}^{*}\right)$ between group $3(3.06 \pm 0.55)$ and groups $1(1.44 \pm 1.32$; $p<0.04)$ and $2(1.34 \pm 1.09 ; p<0.03)$ respectively.

\section{Conclusions}

Our results seem to indicate that green tea only slightly reduces chroma in the darkest colours of the Inspiro ${ }^{\circledR}$ flowable composite, but it does not affect the value and hue.

\section{- Oral Presentation 90}

TITLE: Importance of dark curing phase in color. Preliminary study

AUTHORS: Serrano Cerdá N, Miñano Cerdá L, López Rodríguez A, Chiva García F, Nicolás Silvente AI.

\section{Objectives}

Evaluate the importance of the dark phase of the light curing in the parameters of the color space Cie$\mathrm{L}^{*} \mathrm{C}^{*} \mathrm{~h} *$.

\section{Materials and Methods}

A cavity $6 \mathrm{~mm} \varnothing$ and $3 \mathrm{~mm}$ deep was made on the buccal aspect of 15 molars. They were filled with dentin composite i2 $2 \mathrm{~mm}$ Inspiro ${ }^{\circledR}$ and $1 \mathrm{~mm}$ enamel composite Inspiro ${ }^{\circledR}$ (Axis Dental, Switzerland). They were randomly divided into 3 groups: Group 1: i2 + Bleach; Group 2: Neutral and grupo3 i2 + i2 + Ivory. They were light cured 20" with a LED light (miniLED $\AA$, Satelec) and polished with Enhance/PoGo ${ }^{\circledR}$ (Dentsply) system. The color was measured with a Easyshade ${ }^{\circledR}$ (Vita) spectrophotometer before polishing, after polishing and after 7 days. Samples were stored at $36^{\circ} \mathrm{C}$ in saline physiological solution.

\section{Results}

When comparing the three groups, the were no significance differences between polished and unpolished composite as to the values of $\mathrm{L}^{*}, \mathrm{c}^{*}$ and $\mathrm{h}^{*}$. However, when comparing the differences of $L^{*}, c^{*}$ and $h^{*}$ be- 
tween the just polished and 7 days after polished a significant decrease in composite luminosity ( $\mathrm{L}^{*}$ ) group 3 with enamel Ivory $(11.34 \pm 4.37)$ was observed with respect to the other two groups (Bleach, $5.34 \pm 3.16 ; p$ $<0.025)$ and Neutral $(6.06 \pm 5.20 ; p<0.04)$ and also a significantly increased of hue $\left(h^{*}\right)$ of the group 1 with Bleach enamel $(4.14 \pm 1.26 ; p<0.05)$ and group 2 with Neutral enamel $(4.18 \pm 0.58, p<0.05)$ versus Ivory enamel (1.02 \pm 0.58$)$.

\section{Conclusions}

After polymerization, the darkest color (Ivory) of the Inspiro enamel composite combined with dentin i2 seems to lose brightness and slightly lighter colors of enamel mass seems to increase hue.

\section{- Oral Presentation 91 \\ TITLE: Color perception of students of Den- tistry grade}

\section{AUTHORS: Chykanovskyy V, Guerrero-González M, Santabarbara-Serrano J, Monticelli F.}

\section{Objectives}

The aim of this study is to evaluate the existing congruity in the subjective capture of the dental color on the students of fifth course of odontology and the objective capture with the spectrophotometer.

\section{Materials and Methods}

424 teeth were included in double blind clinical trial. The color measurement was carried out by the students of 5th course with the colors guide VITA Linearguide 3D-MASTER $®$ following the manufacturer's instructions. The color record was effected identifying the unitary color and for dental thirds. As objective reference were used the color records from spectrophotometer SpectroShade ${ }^{\mathrm{TM}}$ Micro. For study of the congruity between the guide Vita 3D master and the students criterion, was calculated the coefficient kappa of Cohen ( $\kappa)$. The statistical analysis was realized with IBM SPSS 22.

\section{Results}

A reasonable congruity is observed in the capture of the color of the unitary tooth $(\kappa=0,34)$ and the middle third $(\kappa=0,21)$, as well as a poor congruity in the incisal third $(\kappa=0,14)$ and cervical third $(\kappa=0,12)$; all statistically significant $(p<0,001)$.

\section{Conclusions}

The election of the correct color of the tooth supposes a challenge, even for experienced clinicians. The students of fifth course of the grade in odontology take better the general color of the tooth and the middle third that the incisal third and the cervical third. The translucency of incisal third and the proximity of the gingival margin to cervical third explain that difference in color evaluation. 


\section{RESINS}

\section{- Oral Presentation 92 \\ TITLE: Characterization of the polymerization of composites using laser speckle patterns}

\author{
AUThORS: Salas M, Pozo AM, Pérez MM, Pulgar \\ R, Lucena C.
}

\section{Objectives}

The purpose of this study was to develop the sequential correlation of laser speckle patterns as a dynamic method to characterize the polymerization reaction of the composites.

\section{Materials and Methods}

The composite Filtek XTE (enamel, dentin and body) at colors A1 and A3 (3M ESPE) was used. Uncured composite was pressed into a mold (1mm high x $8 \mathrm{~mm}$ diameter) and irradiated for 30 seconds with an LED lamp to $1.250 \mathrm{~mW} / \mathrm{cm}^{2}$. Speckle patterns were generated by impining light from a laser diode on the composite, being captured with a CMOS camera. Images were collected at the rate of 16 frames/second for 120s (10s before turning on the lamp, 30s during irradiation and 80 s after shutdown). A region of $200 \times 200$ pixels was selected in each image. To quantify the fluctuation of speckle pattern between each sequential image and calculate the correlation, a custom software was developed in MatLab environment based on the Pearson's correlation coefficient. From these data a representative graph of the correlation based on the time was obtained.

\section{Results}

During registration of speckle contrast, the kinetics of the composite immediately increases as a result of the polymerization reaction, resulting in changes in speckle patterns. It was observed that the kinetics begins immediately after the exposure to curing light and continues, although at different rates after the irradiation conclude.

\section{Conclusions}

The evaluation of sequential laser speckle correlation can be very useful to detect possible differences in polymerization's kinetics of composites according to the type of material, or according to the protocol of polymerization applied. This technique does not requires calibration and has the advantages of being non-contact, non-destructive, and has high temporal resolution.
- Oral Presentation 93

TITLE: Comparison of the degree of conversion of different bulk-fill composites obtained by ATR, FT-IRt and micro-Raman

\author{
AUTHORS: Bolaños Carmona MV, Sánchez Durán \\ D, Benavides Reyes C.
}

\section{Objectives}

Compare the degree of conversion of different bulk-fill resin composites obtained by ATR, FT-IRt and microRaman.

\section{Materials and Methods}

ix bulk-fill resin composites were investigated, including three packable Admira Fusion (Voco), Aura Bulk Fill (SDI) y X-tra Fill (Voco)) and three flowable (Venus Bulk Fill (Heraeus), Filtek (3M), Xtra-Base (Voco)).

Cylindrical molds of $1 \mathrm{~mm}$ diameter and $6 \mathrm{~mm}$ thickness were performed.

Each sample was light-cured from the top as specified by the manufacturer with a LED curing unit Bluephase $20 \mathrm{i}(1200 \mathrm{~mW} / \mathrm{cm} 2)$. For each composite 9 molds were made, which were divided among the three tests: ATR, FT-IRt and micro-Raman. The $0 \mathrm{~mm}$ and $4 \mathrm{~mm}$ slides of each mold were studied and a spectra of the uncured material was also measured(control).

ATR: $600-4000 \mathrm{~cm}-1$. The acquisition time was $10 \mathrm{~s}$ with 10 accumulations.

FT-IRt: $600-4000 \mathrm{~cm}-1$ at $2 \mathrm{~cm}-1$ resolution over 1024 scans.

Micro-Raman: $785 \mathrm{~nm}$ in the wavenumber range 1500$1750 \mathrm{~cm}-1$. The acquisition time was $10 \mathrm{~s}$ with $10 \mathrm{accu}-$ mulations.

All spectral data were analyzed using the same methodology. The two peaks of interest were analyzed: $1607 \mathrm{~cm}-1$ and $1637 \mathrm{~cm}-1$ (area, amplitude, center and FWHM). The percentage DC was calculated for each sample at $0 \mathrm{~mm}$ of depth and $4 \mathrm{~mm}$ of depth.

The means and standard deviations of the analyzed parameters were compared between test, materials and deep with the the Wilcoxon test.

\section{Results}

FT-IRt showed lower values of DC, both areas and amplitudes of the peaks. Differences between $0 \mathrm{~mm}$ and $4 \mathrm{~mm}$ were detected more precisely by micro-Raman. FTIR-ATR obtained DC values significantly higher than those obtained by FTIRt.

\section{Conclusions}

The vibrational spectroscopy method significantly influences the degree of conversion of bulk-fill composites. 


\section{OTHERS}

\section{- Oral Presentation 94}

TITLE: Preclinical assessment methodology of cavity preparations: methacrylate blocks and Simodont ${ }^{\circledR}$ Haptic 3D-VR

\author{
AUTHORS: Aliaga Vera I, Vera González V, García \\ Barbero AL, Hernando Dumaraog B, Pedrera-Cana \\ M.
}

\section{Objectives}

Analyze whether the preclinical practices performed in a dental simulator and in methacrylate blocks detect the manual skills of students over 2 years and obtain a preclinical evaluation methodology for all the parameters that measure Simodont $\AA$ performance (\%) in each of the prepared figures, using the criteria and the evaluation scale of the methacrylate block practice as a predictor (0-10).

\section{Materials and Methods}

Eighty one students who completed the 1st year of dentistry school and entered the 3rd year performed the following tasks; cavity preparation of 3 figures (bar, circle and cross) in Simodont $\AA$ and in methacrylate blocks, the latter being the only one evaluated by the professor. The Wilcoxon signed-rank test was performed to observe differences between the course years in the parameters measured by Simodont ${ }^{\circledR}$ and the evaluation of the methacrylate blocks. Through the predictive techniques of linear regression analysis and a decision tree, the data obtained from both practices were related to each other to obtain models that were applied to Simodont ${ }^{\circledR}$ measure parameters $(0-10)$ used in evaluating the student for each figure.

\section{Results}

There are significant differences between the 1st year and 3rd year in the methacrylate block Grade $(p=0,001)$ and parameters that measure Simodont ${ }^{\circledR}$ performance: Drill Time $(p=0,040)$, PDSLB $(p=0,011)$, PDSLS $(p=0,023), \operatorname{PDSCB}(p=0,045)$. For predicting Simodont evaluation based on the methacrylate block grade Better results $(>$ R2) were attained in the linear regression analysis in all figures and in the 2 courses.

\section{Conclusions}

Both methodologies are able to detect the learning curve of the students, and we are able to evaluate Simodont ${ }^{\circledR}$ practice reliably by linear regression analysis. These results are a first step in establishing the validity of this methodology.

\section{- Oral Presentation 95 \\ TITLE: Efecto de irrigantes in the intracanal cleaning after post space preparation}

\section{AUTHORS: Vázquez Natividad I, Alegre Domingo T, Faus Matoses V, Faus Matoses I, Faus Llacer VJ.}

\section{Objectives}

The aim was to evaluate the effect of different irrigating solutions on smear layer removal and dentinal tubule opening on root canal surfaces after post space preparation.

Materials and Methods

Thirty human single root extracted teeth were endodontically treated. After post-space preparation, specimens were divided into 3 groups and each assigned to an irrigant protocol: group 1, EDTA 17\% 60 sec.; group 2 , EDTA $17 \%$ 60sec. Followed by $\mathrm{CHX} 2 \%$; group 3, maleic acid 7\% $60 \mathrm{sec}$. Specimens were split longitudinally in the labiolingual direction and examined under a scanning electron microscope. They were scored for smear layer removal and dentinal tubule opening at the coronal, middle, and apical thirds of the root canal. Smear layer removal and dentinal tubule opening results were statistically analyzed with the kruskal-Wallis $\mathrm{H}$ test and Mann-Whitney U test $(P<0.05)$

Results

There were no significant differences among the 3 different groups on the removal of debris, sealer and guttapercha and dentinal tubule opening at each third of the root canal surfaces $(P>0.05)$.

Conclusions

The three different post space treatments tested were effective in removal of smear layer of debris, sealer and guttapercha and dentinal tubule opening on the root canal. It may be beneficial for the bonding of the fiber posts.

\section{- Oral Presentation 96 \\ TITLE: Microbiological control of cariogenic biofilms using phosphoric and phytic acids}

\section{AUTHORS: Laguna Soler L, González de Molina Fernández A, Ferrer Luque CM, Baca P, Arias Moliz MT, Ruiz Linares M.}

\section{Objectives}

The aim of this study was to evaluate the ability of phosphoric and phytic acids, alone and with $2 \%$ chlorhexidine, in controlling cariogenic biofilm formed in dentin.

Materials and Methods

Antimicrobial activity of $37 \%$ of phosphoric acid and $1 \%$ phytic acid, alone and combined with $2 \%$ chlorhexi- 
dine, was assayed on mature cariogenic biofilm formed on standardized coronal dentin blocks and subjected to Adenosine triphosphate assay(ATP) and anaerobic culture counts (CFUs). Two specimens were analyzed under confocal scanning laser microscopy (CSLM).The results of bioluminescence and CFUs by groups were analyzed using the ANOVA test and to compare the efficacies of the different protocols the Mann - Whitney test was used. The correlation between the two methods was determined by the Pearson's correlation coefficient.

\section{Results}

A high Pearson's correlation level was obtained between the results of bioluminescence and CFUs counts (r2=0,959). Orthophosphoric acid showed better results in both tests of antimicrobial activity, although no statistically significant differences between groups were found $(p>0,05)$. The application of $2 \%$ chlorhexidine following the orthophosphoric and phytic acids, reduced the metabolic activity of biofilms in $43 \%$ and $34 \%$ respectively. The images obtained with CSLM were consistent with these results.

\section{Conclusions}

The present study shows that, $2 \%$ chlorhexidine improves antimicrobial activity of orthophosphoric and phytic acids, in microbiological control of cariogenic biofilms.

\section{- Oral Presentation 97}

TITLE: Polimedicated institutionalized elderly, prevalence of hyposalivation- quality of life and oral health

\author{
AUTHORS: Sanches C, Marques D, Amaral J, Men- \\ donça C, Mata A.
}

\section{Objectives \\ Sociodemographic characterization of polimedicated institutionalized elderly, to study geriatric salivary pa- rameters and assess the impact of saliva related condi- tions in quality of life.}

\section{Materials and Methods}

The study protocol was approved by the ethics commission and 62 participants from a Home Care Facility were recruited and gave their written informed consent, 18 were excluded for mental illness $(n=44)$. Salivary flow, $\mathrm{pH}$ and buffer capacity were measured, quality of life related questionnaires were applied and Barthel Index evaluated, medical history was collected. Hyposalivation criteria was non-stimulated salivary flow $(\mathrm{NSSF}) \leq 0.1 \mathrm{ml} / \mathrm{min}$ and stimulated salivary flow $(\mathrm{SSF}) \leq 0.7 \mathrm{ml} / \mathrm{min}$. All data analysis was carried out according to a pre-established plan and results presented as mean $+/-95 \%$ CI with $p<0.05$.

\section{Results}

$45.9 \%$ were 85 years or more, $68.9 \%$ women; $61.4 \%$ had Barthel Index $\leq 60$ points (totally or partly independent for daily life activities). mean $( \pm 95 \% \mathrm{CI})$ salivary flow rates and $\mathrm{pH}$ were $\mathrm{NSSF}=0.13 \pm 0.12 \mathrm{ml} /$ min, $\quad \mathrm{NSSFpH}=6.7 \pm 0.6, \quad \mathrm{SSF}=0.59 \pm 0.37 \mathrm{ml} / \mathrm{min}$, $\mathrm{SSFpH}=6.0 \pm 0.9$, these varied between gender but not significantly; $70.7 \%$ had high buffer capacity. Xi-5 mean was $7.77 \pm 2.1$, 48.7\% "occasio-nally" felt their mouth dry, OHIP-14 ranged 0-16 (mean 4.82 \pm 4.9 ); $100 \%$ of the elderly took at least two medications, $34 \%$ had hyposalivation; very weak correlations were found between NSSF and Xi-5 ( $\mathrm{r}=-0.16)$, and NSSF and OHIP$14(\mathrm{r}=0.16)$.

\section{Conclusions}

Hypossalivation prevalence and polimedication levels found within our study are according to previous studies and these values may contribute to an increased dry mouth sensation and lower salivary flow rates. The impact of studied saliva related conditions on elderly quality of life is low.

\section{- Oral Presentation 98 \\ TITLE: Prison management as guarantor of oral health of prisoners}

\section{AUTHORS: Rodríguez Menacho D.}

\section{Objectives}

The prison population in Spain (65.017 inmates in 2014 according to the INE, which represents $0,14 \%$ of the population of Spain), as well as the general population, is beneficiary of the Dental Public Health, depending on the varied legislation that regulates the subject. Nevertheless, it is a specialty, because such individuals have limited right to freedom.

\section{Materials and Methods}

It was performed an analysis on the legislation which regulates the situation, and also the resolutions emitted by the judicial body when the inmates have brought litigation to seek dental care.

\section{Results}

Legislative basis about the subject have been extracted and the analyzed resolutions were grouped into four classes according to the aim: dental public health inside the correctional institution, furlough to obtain dental treatment, economic issues of private dental care, and private dental care inside the correctional institution.

\section{Conclusions}

Conservative dentistry is a pending issue by the Prison Administration. An appeal for dental care does not tend to reach the expectations of the prison population and it involves a vulnerable group that is why the public authorities should offer wider Dental Public Health services. 


\section{DENTAL ALTERATIONS AND DESTRUCTIVE PROCESSES}

\section{- Oral Presentation 99 \\ TITLE: Minimally invasive treatment of eroded upper incisors}

\author{
AUTHORS: Vílchez de la Fuente B, Vílchez Díaz \\ MA, González López S.
}

\section{Introduction}

Dental erosion is a multifactorial process characterized by the dissolution of dental tissues because of dietary imbalances as acidic beverages, high consumption and production of abnormal intrinsic acid (bulimia nervosa, regurgitation ...), and insufficient flow rate of saliva.

Traditionally, the recommended treatment has been full crowns. Currently the focus is more conservative, it is to rebuild the eroded palatal composite and use the vestibular enamel with a minimum preparation to make porcelain veneers (Vailati technique).

\section{Case Report}

Male 27 years, consuming more than a liter of Coke a day, with frequent regurgitations who comes to MOME clinic requesting a cosmetic treatment. Clinical examination shows erosion of the palatal surfaces and incisal edges of the upper incisors with an erosional Index (BEWE) 9. Radiological examination shows no abnormality. Plan treatment consists on dietary recommendations aimed at preventing progression of dental erosion and restorative treatment of incisors where Vailati technique is performed.

After the shelling of the sclerotic dentin surface of the eroded palatal surfaces with a diamond bur coarsegrained, composite reconstruction is performed, using adhesives with prior etching. Next step consist on an bucco-incisal intraenamel preparation for ceramic facets E-max. Once made porcelain veneers, we proceed to its placement with adhesive technique.

\section{Conclusions}

Vailati technique is effective for the treatment of eroded upper incisors.

\section{- Oral Presentation 100}

TITLE: Minimally invasive rehabilitation

\author{
AUTHORS: Díez Deustua R, Villegas Akena M, \\ Cantó Navés $O$. \\ Introduction \\ One of the dental destructive processes that occur most \\ frequently in the general population is known as attri-
}


tion, defined as destruction of tooth tissue by mechanical forces. Today new minimally invasive techniques to rehabilitate these cases are presented, as are the direct composite restorations for a full rehabilitation.

\section{Case Report}

A female patient of 70 years comes to the clinic of the Universidad Internacional de Cataluña with the purpose of change three IV type in the antero-superior classes. After the appropriate radiographic and clinical examination, a marked attrition, causing the destruction of the pattern is diagnosed in the antero-superior restorations.

The proposed treatment is the restoration of the normal anatomy of the dental pieces through direct composite restorations, increasing the vertical dimension enough to restore a correct mutually protected occlusion. The process is initiated by waxing all teeth, giving back the corresponding anatomy. After this phase, transparent silicone keys, which are used with the aim of testing the mock up and our guided restorations, are made. Then a mock-up test where we set the parameters acquired in the mouth based on the wax-up is done. Following the parameters of the wax-up/mock-up, a pre-provisionalization is made for a month and a half, period in which we maintain our provisional to consolidate the new vertical dimension before making the final restorations. After the provisional stage, a rehabilitation through quadrants using silicone keys is performed.

\section{Conclusions}

Rehabilitation with composite keys by silicone is an alternative and conservative treatment for mild to moderate cases of attrition, based on minimally invasive dentistry. One of the advantages of this is its high degree of repairability and reversibility.

\section{- Oral Presentation 101}

\section{TITLE: Minimally invasive complete rehabili-} tation of severe wear: one year follow up

\section{AUTHORS: Darriba IL, González CG, González Mosquera AÑ, Da Silva L, Santana-Penín U.}

\section{Introduction}

Dental erosion is characterized by a pathologic, chronic and localized loss of dental hard tissue. It is caused by intrinsic acids, from the stomach, or extrinsic, due to new habits and lifestyles. Dental erosion is increasingly common in the population, and can affect any tooth surface, although it is more common in the palatal surfaces of anterior-superior teeth and occlusal surfaces of the lower molars. Dental erosion has functional, esthetic and biological consequences on dental health. Previously, rehabilitation required root canal treatments and full crowns. However, advances in adhesive dentistry, allow more conservative approach preserving the remaining tooth structure.

\section{Case Report}

A case of a patient with severe wear dentition due to erosion by frequent vomiting associated with stress is described. A conservative approach based on adhesive techniques with minimal tooth preparation was performed. Treatment was planning based on a diagnostic wax-up increasing the vertical dimension. Direct and indirect composite, and lithium disilicate restorations were performed following the three-steps technique. In addition it was necessary to crown lengthening in the anterior-superior sector to level the gingival margins. After one year of follow up there were no clinical complications.

\section{Conclusions}

Advances in adhesive dentistry allow aesthetic rehabilitations with minimally invasive techniques. Although adhesive approach simplifies clinical and laboratory procedures, these rehabilitations are still a challenge due to the severe tooth destruction. Even though there are no long term studies on the success of full mouth adhesive rehabilitations, this approach should be used in cases of severe tooth wear, because it is less destructive and not preclude from future treatment with conventional crowns.

\section{- Oral Presentation 102 \\ TITLE: Management of severe dental wear with a total adhesive rehabilitation}

\section{AUTHORS: Guinovart J, Jane L, Roig M.}

\section{Introduction}

Dental wear, being moderate or severe, is a common pathology in the dental practise. Whenever we face this kind of scenarios, the first step in developing a treatment plan is to diagnose the cause of the wear. Then, we must evaluate the wear severity. It is not difficult to find that the vertical dimension of occlusion may have decreased and supraeruption of alveolar process segments may have occurred, increasing the amount and complexity of the restorative treatment.

Thanks to improved adhesive techniques the indications of crowns has decreased, allowing less invasive approaches, preserving dental structure and decreasing the chance of having dental sensitivity or endodontic needs.

\section{Case Report}

We would like to share with you a case of a patient who presents teeth wear due to a severe attrition which by the use of provisionals and definitive composite resin restorations was rehabilitated achieving an esthetic and specially functional result. 


\section{Conclusions}

In cases of dental wear the predictability of the treatment plan will be determined by the wear severity and by the presence or abscense of sound enamel. If the primary cause of the dental wear is attrition, the prognosis will depend on how well the occlusion can be managed during the provisional phase and the posterior control once definitive restorations are placed.

\section{- Oral Presentation 103}

TITLE: An original method for the recovery of the vertical dimension in cases of complete overbite a purpose of a clinical case

AUTHORS: Navajas Nieto C, Navajas Nieto JM, Travesí Fernández A.

\section{Introduction}

Full overbite malocclusions produce serious destruction of the occlusal surfaces, altering chewing, affecting the health of the pulp and compromising the permanence of the tooth.

\section{Case Report}

Adults 40 years of age, having microdontia and complete overbite. The microdontia was treated in his teens by feldspathic ceramic veneers that allowed partially recuperarar dental aesthetics

The destruction of the crowns of molars and premolars for its sobremordía requires orthodontic treatment.

We present a simple and original method of recovering the vertical dimension and occlusal tooth morphology, as a temporary treatment and support orthodontic treatment within a multidisciplinary context

\section{Conclusions}

The use of direct composites on occlusal surfaces restores lost occlusal morphology and proper vertical dimension allowing the orthodontic treatment of overbite.

\section{- Oral Presentation 104}

TITLE: Posterior rehabilitation for the chronic bruxism: aesthetic planning

AUTHORS: Villegas Akena M, Díez Deustua R, Jané L, Ortega J.

\section{Introduction}

Commonly, aesthetic dentistry is misconceived as cosmetic dentistry, the latter being a branch of mainly selective treatments, in other words, treatments made to beautify what is already sane. Nevertheless, Aesthetic Dentistry is not a synonym, since it sets the original or ideal beauty as the model guide to planify the treatment of something that is not sane.

On the other hand, bruxism is one of the main diseases that affect hard tissues and, in long term, soft tissues of the oral cavity. In several occasions, this condition has been underestimated and no previous analysis previous to a planification is made, and, as a consequence, the treatment fails.

\section{Case Report}

Fifty-year old patient arrives to the clinic with a chief complaint of not being able to chew properly due to absence of posterior teeth. Patient is a chronic smoker and refers being a sleep and awake bruxist.

When exploring, absence of the whole posterior sector in the first quadrant is found, along with the two premolars in the second quadrant having as a consequence an unbalanced occlusion.

The treatment begins with the assessment of the bruxism patterns and a digital smile design. Having this as a reference, a diagnostic wax up is performed and the mock up afterwards. After this, the restoration is developed.

\section{Conclusions}

A comprehension of the main cause of the disease is crucial, along with the intentions to cure and not only reconstruct. Esthetic planification means to restore with the reference of what is the ideal, and when we say "ideal" is because it is functional.

\section{- Oral Presentation 105}

\section{TITLE: Unilateral fusion of mandibular inci-} sors: case report

AUTHORS: Jiménez Sánchez MC, Tarilonte Delgado ML, Martín González J, Murillo Benitez M, Segura Egea JJ.

\section{Introduction}

Fusion is a dental development anomaly that affects the number, size and shape of the tooth. It is caused by disturbances during embryonic development, and its etiology is normally unknown.

\section{Case Report}

A 32 male requested dental assistance because displacement of the mandibular midline. Clinical exploration revealed the presence of only 3 incisors in the lower arch: a central and lateral left incisors of normal shape and size, together with, on the right side, a tooth larger than normal with anomalous coronal shape. The strange tooth showed a buccal groove that divided the crown longitudinally and which was also visible on the palatal surface. The tooth has no cavities and pulp vitality tests, periodontal probing, and mobility were normal. Periapical radiograph showed a single root of larger size 
than normal, with a large pulp chamber and a single root canal; no signs of periapical pathology were evident. The teeth count was necessary to establish the final diagnosis: the anomalous tooth had to be counted twice to achieve the normal number of teeth. Therefore, the diagnosis of unilateral fusion of right central and lateral incisorswas established.

\section{Conclusions}

The scientific literature names these cases as "double tooth". The diagnosis implies the differentiation with a case of macrodontia and incisor agenesis. However, in this case, the abnormal morphology of the crown of the affected tooth suggests the diagnosis of fusion between both right mandibular incisors. The treatment options will depend on the affected morphology of the dental arch, the occlusion pattern and aesthetic requirements.

\section{- Oral Presentation 106}

\section{TITLE: Aesthetic management of anterior teeth with hypocalcified enamel defects}

AUTHORS: Sena Herrero O, Agulló Vidal C, Marques H, Vallés Rodríguez M, Ramírez Sebastià A, Jané Noblom L.

\section{Introduction}

Management of hypocalcified enamel defects is a challenging aspect of aesthetic dentistry. Some of these defects can be treated with microabrasion if they affect the enamel surface only. However, if the defect spans the thickness of the enamel to the dentin-enamel junction, microabrasion will not be effective. Removal of these unsightly defects mechanically with a bur and composite restoration may be the treatment of choice.

\section{Case Report}

A 22-year-old male patient was referred to us presenting a discoloration in 2.1. A diagnosis of enamel hypoplasia was made. Due to the extensive depth of the lesion, removal and composite restoration was indicated.

Enameloplasty was performed with diamond burs. Color was recorded before rubber dam isolation. The enamel surface was acid etched (37\% phosporic acid) and a two-component adhesive system (Optibond FL, Kerr) was applied. The cavity was filled with microhybrid composite (CeramX Duo, Dentsply). Polishing was performed with fine diamond burs and the final glossing was achieved using aluminum oxide paste (Shiny $\mathrm{C}$ Enamel Plus, Micerium) with a felt wheel.

\section{Conclusions}

We can conclude that the proposed approach is a simple therapeutic option, which can provide a long-lasting satisfactory aesthetic appearance on teeth affected by white enamel discolorations.

\section{- Oral Presentation 107 TITLE: Internal and external bleaching}

AUTHORS: Marques H, Senna O, Agulló C, Vallés M, Ramírez-Sebastià A, Jané L.

\section{Introduction}

Discoloration of an anterior tooth may have a significant social impact. The chosen treatment should be minimally invasive and should not compromise future restorative options. Bleaching of non-vital teeth is a low-risk treatment that improves esthetics. There are several techniques for bleaching non-vital teeth: walking bleach technique, in-office bleaching and inside/ outside bleaching technique. The products available are the hydrogen peroxide, carbamide peroxide and sodium perborate. The use of hydrogen peroxide in a high concentration is caustic and if heated can increase the possibility of cervical root resorption. In December of 2010 sodium perborate was forbidden in Europe because of its toxicity. The inside/outside bleaching technique uses carbamide peroxide and the risks of cervical root resorption are less than with the walking bleach technique with a high percentage of hydrogen peroxide.

\section{Case Report}

A 40 year old patient presented a severe yellow-brown discoloration and an old composite restoration of tooth 2.2. The patient mentioned no history of trauma and several previous attempts of external bleaching without success. After performing vitality tests and a periapical $\mathrm{X}$-ray the diagnosis of pulp necrosis with apical periodontitis was established. It was decided to perform the endodontic treatment and an inside/outside bleaching technique with a $10 \%$ carbamide peroxide gel (NiteWhite, Philips Zoom) followed by a direct composite restoration (Filtek ${ }^{\mathrm{TM}}$ Supreme XTE, 3M).

\section{Conclusions}

Pulp necrosis discoloration can usually be bleached intracoronally with good and predictable results. The inside/outside technique is conservative, safe and effective. It allows the use of small concentrations of hydrogen peroxide and therefore reduces the possibility of cervical root resorption.

\section{- Oral Presentation 108}

TITLE: Minimally invasive aesthetic restoration: internal bleaching and direct composites

\section{AUTHORS: Santos Lavoura F.}

\section{Introduction}

In the anterior region, discoloured teeth can result in a considerable esthetic problem. In contrast to crowning or veneering, whitening of teeth is relatively non- 
invasive and conserves more dental hard tissue so it is a good opcion to take in acount when we want to improve aesthetics. Direct composite restourations also allow clinicians to realize restorations on a high esthetic level while being minimally invasive, affordable to patients, and long lasting.

\section{Case Report}

A 30 years-old patient came to the clinic of Universidad Internacional de Cataluña with complaints of dissatisfaction about the appearance of his teeth, such as darkening and chipping of the incisal edge of his central incisor. The patient presents a previous endodontic treatment in the upper central righ incisor done 10 years ago which results in a color alteration. The lateral incisors also have infiltrated restorations that will be replaced for new ones using a direct composite tecnhique in order to achieve better esthetic and function. So we decided to perform internal bleaching in 1.1 with Opalescence Endo and direct aesthetic restorations with nano-hybrid composite IPS Empress Direct from Ivoclar.

\section{Conclusions}

In this clinical case we demonstrate that using very conservative tecnhiques we can improve the esthetic of our patients.

\section{- Oral Presentation 109}

TITLE: A combined mimimal invasive treatment of white enamel spots

AUTHORS: Llosa Mariat Y, García García C, Araujo E, Gomes G, Luengo Capilla MA, Fuentes Fuentes MV, Ceballos García L.

\section{Introduction}

White spots lesions can represent a severe esthetic alteration. Dentists must draw on the most conservative techniques available in order to solve these situations. Resin infiltration is considered a relatively simple and microinvasive technique, since only a minimal portion of enamel is removed.

\section{Case Report}

An 18-year-old woman came to the post-graduate course of Expert in Esthetic Dentistry at URJC dissatisfied with her dental esthetics. She had white developmental enamel lesions on the anterior teeth and surface defects in teeth 11 and 21, probably created by brackets removal. Firstly, a home-bleaching was proposed with 10\% carbamide peroxide (Opalescence 10\%, U1tradent) for 4 weeks to diminish the colour difference between the white spots and the enamel. After that, a resin infiltration treatment with ICON smooth surfaces (DMG) from teeth 13 to 23 was performed, according to manufacturer's instructions. Twenty-four hours later, enamel defects were restored using resin composite
(Light Enamel, GC Essentia). Finishing and polishment was carried out in another session, once we confirmed the resin composite selection was correct.

\section{Conclusions}

The combination of minimally invasive techniques such as bleaching and resin infiltration can be satisfactory for the treatment of white enamel lesions.

\section{- Oral Presentation 110 TITLE: Treatment of white spots lesions}

AUTHORS: San José Vazquez del Rey A, Costas Soto A, Rosel Gallardo E, Otero Ávila A, Wyszkowski Canal M.

\section{Introduction}

The appearance of white spots is a multifactorial etiology. The main causes are: incipient caries, fluorosis, enamel hypoplasia, intake of drugs during the mineralization of enamel or trauma.

The aesthetic compromise is higher when these spots appear in the previous sector, being often the reason for consultation.

There are different treatment options and we must choose that which suposse lower biological cost to the patient. Treatment depends on the depth of the lesion. So far, microabrasion had become the reference treatment, in combination with direct technique using composite and / or whitening. However, removal of the affected tissue is a more invasive treatment but necessary in the case of white deeper spots or when treatment with resins infiltrative is ineffective.

The use of resins infiltrative therefore becomes the most conservative treatment option. The treatment strategy in this case is not based on the removal of the affected tissue, but infiltration hydrophobic resin prior application of hydrochloric acid in surface permeable and thus make the enamel surface.

This technique erosion - infiltration also have been described for the treatment of incipient caries and the treatment of white spots and hypoplastic lesions fluorosis.

\section{Case Report}

We present a series of cases treated with ICON $®$ (DMG) following the protocol indicated compared with clinical cases treated with direct composite technique of patients attending the Master of Aesthetic Dentistry Multidisciplinary demanding an improvement in the previous sector.

\section{Conclusions}

Treatment by infiltrative resins gives a satisfactory solution in white spots and resolution significantly reduce the extent of injuries. In cases where resolution is not possible by air abrasion, we consider infiltrative tech- 
niques and reconstruction with direct composite technique as a second treatment option.

\section{- Oral Presentation 111}

TITLE: Protocol of anterior dental restorations. Alterations in the enamel

\author{
AUTHORS: Arribas Nieto I, Guinovart J, Fenoy Il- \\ lacer P, Jané Noblom L.
}

\section{Introduction}

Minimally invasive approaches are generally established as the most desirable strategies in medical treatments. In dentistry, the preservation of dental tissue is nowadays one of the main goals of the the dentist.

Restorative dental treatment of patients with hipomaduration and hipocalcification of the enamel remains a challenge even today. Dependence on the stage of the defect we can approach with different techniques including Fluorclohidric acid,microabrasion or ma-croabrasion.

The treatment approach is multidisciplinary and includes action of several dental disciplines such as restorative, orthodontic and prosthetic dental.

\section{Case Report}

Case report of a 27 years old patient was referred to the Aesthetic and Prosthodontic Department of International University of Catalunya. Her chief complain was to improve her aesthetic in the anterior zone.

We do know that to achieve an excellent result in anterior zone like getting a perfect shape,color and texture is not easy. We not only need manual ability, we do also need the knowledge of general principles and techniques of restorative therapy.

The ideal and also simpler technique is the 2-layer approach; however, it requires dentin and enamel masses to optimally imitate natural enamei and dentin, which unfortunately is rarely the case.

In this case we apply the conventional stratification technique,will be describe with more detail

\section{Conclusions}

With a good protocol we can solve this cases and achieve optimal results.

\section{- Oral Presentation 112 \\ TITLE: Restoring the dental aesthetic}

\section{AUTHORS: Gallart Manchón P, Bastús Bachs C, Espona Roig J, Jané Noblom L.}

\section{Introduction}

Nowadays we are living in a society where there is huge demand of aesthetics by patients, where there are also high expectations, and looking for the lowest price. That's the reason why always it is a challenge for the clinician when he faced cases in the anterior area, furthermore, in cases of devitalized teeth, when there is no consensus in the literature about the ideal approach.

\section{Case Report}

In this case we follow a minimally invasive treatment for the anterior region for a right first central incisor, devitalized with a different colour, inadequate proportions, and the adjacent teeth with fillings class III desadjusted. Therefore, the patient came to our office looking for an aesthetic solution for that problem.

On the first visit was done the anamnesis, cast models, facebow and bite registration for subsequent assembly in articulator, intraoral and extraoral photos for the case study were taken and radiographic study was performed.

We present different treatment options to the patient, but due to the economic limitations of the patient, the case was solved with a direct composite restoration (Inspiro, Edelweisse), reaching the desired aesthetic result for the patient.

\section{Conclusions}

Despite not finding a consensus in the literature for the approach in these types of cases, we offered to our patient an optimal result in terms of aesthetic expectations, through a minimally invasive treatment.

\section{- Oral Presentation 113}

TITLE: Dental trauma, aesthetic management: case report

\section{AUTHORS: Serrat Baron R.}

\section{Introduction}

High expectations and very high aesthetic demands from our patients make the dental trauma a challenge to face frequently. It is therefore presented in this case, a clinical approach following the principles of minimally invasive dentistry, for planning and treatment in the anterior area.

\section{Case Report}

Woman, twenty-nine years old, who came to the University Dental Clinic at the International University of Catalonia, referring be complexed for having a very dark tooth. She had the right maxillary central incisor with an over fifteen years root canal treatment due to trauma, presenting high degree of discoloration, and a Class IV unaesthetic composites in both central incisors and the upper right lateral.

After a complementary, radiographic and aesthetic study, was decided to perform a technique of internal and external tooth whitening using Carbamide Peroxide $16 \%$ for ambulatory use, aiming optimal substrate. Subsequently, the composite restorations were replaced for better aesthetic and functional integration. 


\section{Conclusions}

The combination of internal and external tooth whitening help us to achieve, in most cases, a favorable aesthetic appearance. The use of direct composites can be a tool to achieve the expectations of our patients following the principles of minimally invasive treatments. A correct diagnosis and treatment plan, makes possible the obtention of a predictable, optimum and satisfactory results.

\section{- Oral Presentation 114}

TITLE: Partial removal of carious dentin. A case report

AUTHORS: García García C, Llosa Mariat Y, Araujo E, Gomes G, Luengo Capilla MA, Fuentes Fuentes MV, Ceballos García L.

\section{Introduction}

Traditionally, treatment of carious lesions involves the complete removal of soft and infected dentin before restoration. At present, scientific evidence shows that incomplete removal of the infected and softened dentin near the pulp, whenever marginal seal is ensured by adequate filling, allows the pulpo-dentinal complex to remain vital and to arrest the carious lesion.

\section{Case Report}

A 34-year-old female attended the Expert in Esthetic Dentistry Course at Rey Juan Carlos University for a dental checkup. Although visually no carious lesion was observed, in bitewing radiographs a carious lesion affecting the deep third of dentin was detected in distal surface of 15 . The tooth pulp vitality was positive, with absence of spontaneous pain and no discomfort percussion. The treatment proposed was a partial removal of decay and tooth restoration with composite resin. Once the absolute isolation with rubber dam was performed, the carious tissue at the margins and walls of the cavity was removed with a round tungsten-carbide bur in a low speed dental handpiece. A resin-modified glass ionomer was placed on the cavity floor (Vitrebond Plus, $3 \mathrm{M}$ ESPE) and the tooth was restored with Scotchbond Universal adhesive (3M ESPE) and the composite resin Filtek Supreme XTE (shades A2D and WE; 3M ESPE), with an incremental technique.

After 3 months of restoration, the tooth remained vital and without symptoms.

\section{Conclusions}

The partial removal of carious dentin in permanent teeth with deep cavities and in absence of irreversible pulpitis is an alternative to avoid pulp exposure and more invasive treatments.

\section{INDIRECT TECHNIQUE -CAD/CAM}

\section{- Oral Presentation 115 \\ TITLE: Posterior tooth restoration with endo- crown: a case report}

AUTHORS: González Acosta L, Castelo Baz P, Bahillo Varela J, Martín Biedma B, Varela Patiño P.

\section{Introduction}

When considering the endodontically treated teeth restoration, we have a wide range of different treatment options. The current approach is towards a more conservative dentistry using adhesive techniques, attempting to conserve as sound tissue as possible, leaving full coverage crowns to cases were there is great loss of dental structure.

These procedures of post-and-core and total crowns may be invasive both to the crown and the root, and in case of failure the tooth is exposed to a higher risk of irreversible fractures during retreatment procedures.

With adhesive restoration techniques, we can keep as much sound structure as possible, giving the tooth back its mechanical, functional and esthetic properties.

In the choice of the type of restoration, it is important to evaluate the sound tissue and the location of the margin. In cases of deep margins with invasion of the biologic width, a crown lenghtening surgery is indicated.

\section{Case Report}

30-year-old woman attended to the dental clinic with periodontal pain in the second quadrant. During the clinical examination, a large silver amalgam restoration in tooth 2.6 was observed. In the bite-wing x-ray, the deep restoration margin in distal was evidenced, with possible invasion of the biologic width. The need for endodontic retreatment was discarded due to the lack of periapical signs and symptoms.

A crown lenghtening surgery was planned in order to reestablish the periodontal health, and the type of restoration chosen was a composite endocrown (Lava Ultimate).

\section{Conclusions}

The development of the adhesive techniques has allowed the evolution to more conservative treatments. Indirect restorations with endocrowns may be the treatment of choice in this type of cases.

\section{- Oral Presentation 116}

TITLE: Indirect restoration in endodontically treated teeth. Conventional technique Vs. CADCAM

AUTHORS: Álvarez-Maldonado N, Torres Muñoz A, Roldán Cubero J. 


\section{Introduction}

The reconstruction of endodontically treated teeth still remains controversial in Dentistry. Thanks to new technologies and adhesive techniques, more conservative alternatives are introduced, in opposition to yesterday's designs, when preparations were made based on the material properties and macro retention requirements.

In the case of a posterior tooth with an endodontic treatment and great loss of structure, there are different treatment options: direct composite restoration, indirect restoration (onlay / overlay / endocrown ) or full coverage crown.

In these cases, direct restorations have limitations such as the control of polymerization shrinkage, the conversion factor, and technical and occlusion management among others, that make them contraindicated in large cavities. In addition, indirect restorations allow us to restore and Protect the remaining tooth structure, being conservative and increasing resistance to fracture.

\section{Case Report}

We report a case of a 14 years old patient, who comes to the University Dental Clinic of the International University of Catalonia in need of an indirect reconstruction of his first lower molar endodontically treated. Two resin overlays are made by conventional indirect stratification technique and CAD-CAM for comparison in aesthetic aspects, anatomy and mimetization.

\section{Conclusions}

Advances in $\mathrm{CAD} / \mathrm{CAM}$ technology greatly simplify work in the dental office, reducing chair times and making the dentist's work easier, but still needs to be developed further to achieve full mimetization of the restoration in terms of anatomy and characterization.

\section{- Oral Presentation 117}

TITLE: Endocrown, alternative to full coverage crowns: a case report

\author{
AUTHORS: Sánchez Blanco I, Sauco Márquez JJ, \\ Montero Miralles P, Segura Egea JJ, Fernandez Pri- \\ eto $\mathrm{C}$.
}

\section{Introduction}

Traditionally, we have chosen full coverage crowns to restore endodontically treated teeth with large coronal destruction. Another treatment option in order to restore these teeth is an endocrown. This monolithic, ceramic adhesive restoration tries to be conservative, adapting itself to the remaining dental tissue. The tooth preparation of endocrowns is simple and less traumatic than for full coverage crowns and the supragingival margin aids to preserv periodontal health. The design of this type of restoration also helps to distribute and dissipate forces to prevent tooth fracture.

\section{Case Report}

A case of a premolar 1.5 with extensive loss of coronal structure is presented. Root canal treatment was performed and a lithium disilicate endocrown was chosen to restore it. Dental preparation was conservative and an intraoral digital scanner was used to perform a dental impression. The design of the endocrown was done on the digitalized impression and this design was subsequently milled on a lithium disilicate block. After the preparation, the endocrown was cemented obtaining good fit and aesthetics.

\section{Conclusions}

Endocrowns fits perfectly with the concept of biointegrated prosthesis, restoring aesthetic and function of the tooth, conserving as much tooth structure as possible. $\mathrm{CAD} / \mathrm{CAM}$ technology helps us to simplify procedures, minimizing errors and ensuring a clinically acceptable fit of the restoration.

\section{- Oral Presentation 118}

TITLE: Indirect endocrown with composite resin. Clinical technique

\author{
AUTHORS: Álvarez Correa C, San José Vázquez \\ del Rey A, González Villafranca P, Conde Pais J, \\ González López S.
}

\section{Introduction}

The endocrown is a restorative option for endodontically treated teeth that can be considered a reliable alternative to full crown restoration, especially in nonvital posterior teeth with large MOD cavities but having enough buccal and lingual enamel available for a stable and durable adhesive cementation.

\section{Case Report}

A 45-year-old patient came to the clinic of the University of Granada with a deep MOD carious lesion in relation to 4.7 for that reason we chose a restoring technique with total cusp coverage (indirect endocrown with composite resin).

After root canal treatment a direct obturation was carried out using composite then the occlusal surface was reduced just below the maximum dental contour to achieve a restoration volume of 2-3 $\mathrm{mm}$. In the center of the restoration a pseudo-pulpar chamber was drilled with divergent walls that would serve merely to ensure the correct insertion and settling of the restoration. An impression was taken with alginate and it was poured whit the silicone by molding of GradioSo Inlay System. The modeling technique consisted of placing little balls of composite of the size and height of each cusp. The anatomic details were then marked using a PK-Tomas instrument and then each side of the restoration was polymerized. Finally endocrown was cemented used dual 
adhesive cement, the occlusion was adjusted, and we proceeded to final polishing.

\section{Conclusions}

Endocrowns made with composite offer full occlusal coverage with high resistance, a homogeneous distribution of biting forces during function, and excellent esthetics.

\section{- Oral Presentation 119}

TITLE: Restoration of a root-filled tooth with the Cerec Cad-Cam chairside system

AUTHORS: Gallardo Rodríguez M, Martín Jiménez M, Fernández Pino N, Jiménez Fernández M, Segura Egea JJ.

\section{Introduction}

Cad-Cam chairside systems offer to patients and dentists the possibility of restoring teeth with inlays, onlays, veneers or crowns in a single session. A case of restoration of an upper central incisor using a ceramic crown manufactured with the CEREC Cad-Cam chairside system is reported.

\section{Case Report}

A 54-year-old patient seek for dental urgent assistance because uncemented crown on an upper incisor. Clinical examination shows the uncemented metal-ceramic crown on tooth 21. Periapical radiographic examination revealed that tooth was had endodontically treated, with subobturation of root canals, being evident a great radiolucent osteolytic periapical lesion. To assess the dimensions of the periapical lesion, a CBCT scan was prescribed, revealing a large periapical bone defect. Endodontic retreatment was proposed, together with reconstruction with fiberglass post and composite resins, and the placement of a temporary crown. Root canal treatment was carried out in two sessions. In the first one, removal of previous filling material was performed, followed by root canal instrumentation using Mtwo files up to 40/04. Disinfection was carried out with $4.2 \%$ sodium hypochlorite. A calcium hydroxide dressing was put into the root canals as temporary medication. In the second session, root canal treatment was finished and the tooth restored with a temporary crown. After six-months follow-up, great improvement of the lesion was observed. Then, the tooth was definitively restores with a ceramic crown made with the CEREC Cad-Cam chairside system.

\section{Conclusions}

Cad-Cam system, such as CEREC (Dentsply-Sirona), offer an easy alternative for the chairside restoration of root filled tooth using metal-free restorations, as ceramic crowns, providing a high aesthetic and allowing very conservative carved for the dental structures.

\section{- Oral Presentation 120}

TITLE: Endodontic treatment of a calcified maxillary right premolar and restoration with a chairside Cad-Cam manufactured ceramic endocrown

\author{
AUTHORS: Rodríguez Núñez M, Martín Jiménez \\ M, Saúco Márquez JJ, Segura-Egea JJ.
}

\section{Introduction}

Chairside indirect restorations manufactured with CadCam system are suitable for the restoration of root filled teeth. Vita Enamic hybrid ceramic and Vita Suprinity vitreous ceramic, reinforced with zirconium dioxide lithium silicate, provide strength and aesthetics. A case of restoration of a root filled tooth using an indirect ceramic endocrown made with Cad-Cam is presented.

\section{Case Report}

A 56-year-old woman seek for dental assistance because of pain in 2.5. Clinical examination revealed the presence of a composite restoration in the affected tooth. Percussion and palpation were positive and the response to vitality tests was negative. Radiographic examination showed a radiolucent periapical lesion and the presence of a crown restoration with two pins. Irreversible pulpitis with symptomatic apical periodontitis were diagnosed, and root canal treatment was indicated. Cavity access was difficult because pulp chamber had undergone calcic metamorphosis. CBCT was carried out to better determine root canal permeability. After the use of ultrasonic device (Start-X2), root canal glide path was achieved. Root canal instrumentation was performed using 25.07 Mtwo file. The disinfection protocol comprised $\mathrm{NaOCl} 5 \%$ and EDTA $17 \%$. Then, root canal obturation was carried out using the Elements Systems unit. After performing root canal treatment, SDR composite was used to stabilize the cavity margins. OmniCam was used to scan the cavity shape, and to design and manufacture in hybrid ceramic (Vita Enamic) a meso-structure with $3 \mathrm{~mm}$ intracanal extensions, to increase retention. After milling and cementation, the stump was carved. Then, a new scan was carried out, and the designed crown was manufactured with glass ceramic (Vita Suprinity) A3.5. Finally, the indirect restoration was cemented.

\section{Conclusions}

Chairside indirect ceramic restorations (inlay, onlay, endocrown) performed using Cad-Cam systems, are a quick, aesthetic, and ideal choice for the restoration of endodontically treated teeth. 


\section{- Oral Presentation 121}

TITLE: Indirect adhesive restorations with hybrid materials in posterior

\section{AUTHORS: Fernández Fernández A.}

\section{Introduction}

In recent years, advances in CAD-CAM technology; the constant improvement of the physical, chemical and mechanical properties reconstruction materials; advances in high bond strength in adhering tooth; the increase in the supply of many materials for indirect restorations and demand by patients increasingly conservative and aesthetic treatments. They are doing that in our daily practice, perform less invasive dental preparations "restitutio ad integrum" of the tooth. Thus avoiding unnecessary removal of tooth structure allowing maximum tissue preservation and fulfilling biomimetic concept.

\section{Case Report}

This communication will be focused on the use of hybrid materials as the material of choice for this type of reconstruction.

We will give an overview of the different types of existing choice, the design of the cavity preparation, the protocol adhesive cementation of restorations hybrid materials. I will show five clinical cases resolved with different types of hybrid materials. The chosen hybrid materials are infused ceramic and polymer resins nanoceramics.

\section{Conclusions}

Until recently, the use of inlays / onlays / overlays in daily practice was almost obsolete.

The currently existing materials for making these restorations are ceramic materials and hybrid materials. Each with a number of properties and advantages over the other.

In this paper we try to clarify the different types of hybrid materials we choose, with their advantages and disadvantages. The indications for these restorations to our patients.

Hybrid materials have a flexural strength, modulus of elasticity and very similar hardness to dentine. In the case of breakage, they have easy intraoral repair. For these properties, among others, they are a good choice for material indirect adhesive restorations.

\section{- Oral Presentation 122}

TITLE: Endodontic and restorative treatment, one concept

AUTHORS: Cobos González S, Torres González I, González López S, Rosel Gallardo E, Muñoz Puerto AB.

\section{Introduction}

One of the main aims of restoration for the endodontically treated tooth is to achieve an effective marginal seal due to the fact that corono-apical leakage is one of the most common causes of endodontic failure. Therefore it is necessary to design a restoration that ensures marginal sealing to avoid filtration and it protects the remaining tooth tissues from fracture. Adhesive restorations with full occlusal coverage such as endocrown represent an excellent option for treatment.

\section{Case Report}

Male patient, 31 years old, came to the clinic of the University of Granada with a deep carious that affected the pulp in the lower left first molar (3.6). Root canal treatment was carried out with rotary endodontic instrumentation (ProTaper next system) and thermoplastic obturation (Thermafil). Then a direct composite filling was made and the occlusal surface was reduced just below the maximum contour of the tooth to create a restoration with a full occlusal coverage of $2-3 \mathrm{~mm}$ thick. Futhermore, a cavity with divergent walls was prepared to ensure proper insertion and sealing of the restoration. An endocrown was built up with a semi-indirect technique and it was finally bonded with adhesive techniques.

\section{Conclusions}

No post adhesive restoration with full occlusal coverage are a good approach for restoring endodontically treated molar. Endocrowns are a successful choice to achieve marginal sealing and protect the residual tooth tissues.

\section{- Oral Presentation 123 \\ TITLE: Cracked tooth syndrome. Conservative restoration treatment with endocrown}

AUTHORS: Wyszkowski Canal M, González López S, Conde Pais J, González Villafranca P, Cobos González S.

\section{Introduction}

The Cracked Tooth Syndrome was first described by Cameron in1964 as an incomplete fracture in a subsequent vital tooth that compromises the dentine and, occasionally, the pulp. It appears most frequently between the fourth and sixth decade of life, regardless of gender, with the lower molar being the 2nd most affected tooth by its proximity to the TMJ and the type of load that it receives. Due to its high incidence, the diagnosis of a cracked tooth is extremely important: a correct anamnesis, together with a clinical and radiological examination, are essential, with the Bite Test as the great indicator of the presence of fissures in which pain occurs with the release of masticatory pressure. Treatment depends on the state of the fissure, knowing that these teeth have an uncertain prognosis. 


\section{Case Report}

A 60-year old female patient who comes to Máster en Odontología Multidisciplinar y Estética with pain when chewing with \#37 that shows an old Class I restoration with an obvious fissure in the occlusal surface that extends distally. Diagnostic tests reveal: positive on percussion and depth probing, sensitivity to cold and a positive response to the bite test. Radiographically, we find a slight widening of the periodontal ligament. Due to the presence of pulpal pathology, the treatment of choice was a root canal with a subsequent indirect Endocrown restoration.

\section{Conclusions}

A 60-year old female patient who comes to Máster en Odontología Multidisciplinar y Estética with pain when chewing with \#37 that shows an old Class I restoration with an obvious fissure in the occlusal surface that extends distally. Diagnostic tests reveal: positive on percussion and depth probing, sensitivity to cold and a positive response to the bite test. Radiographically, we find a slight widening of the periodontal ligament. Due to the presence of pulpal pathology, the treatment of choice was a root canal with a subsequent indirect Endocrown restoration.

\section{- Oral Presentation 124}

TITLE: Lithium disilicate veneres in anterior teeth with tetracycline stains

\section{AUTHORS: Abadi K, García Bravo M, Guerrero Ji- ménez F, Rodríguez Pérez M, San José Vázquez del Rey A.}

\section{Introduction}

Teeth with tetracycline stains can be a challenge for the clinician. The appropriate treatment option in each case will depend on the severity of the staining. Porcelain veneers, within a multidisciplinary approach to the case, are the treatment of choice when such stains are more serious.

\section{Case Report}

A 56 year-old woman patient, who attends the Máster en Odontología Multidisciplinar Estética at the Faculty of Dentistry (University of Granada) asked an improvement in her dental aesthetics, because she had severe thirddegree tetracycline stains. As a first treatment option, direct composite veneers (Amaris VOCO ${ }^{\circledR}$ ) were performed, resulting not so satisfying the patient expectations; therefore, and after making a re-evaluation of the case, lithium disilicate veneers were made after crown lengthening of the anterior sector.

\section{Conclusions}

The actual techniques and materials allow us to achieve results both aesthetically and functionally excellent.
Ceramic restorative systems have advanced esthetic results specially in the anterior sectors. It is important to know the indications of the materials and make a correct diagnosis to proceed to the choice of the most suitable material.

The lithium disilicate is a ceramic material which got high strength and aesthetics.

\section{- Oral Presentation 125 \\ TITLE: Restorative and endodontic treatment: onlay with CAD-CAM}

\section{AUTHORS: Tarozzi Gorris A, Bruño Fabrega M, Vallés Rodriguez M, Jane Noblom L.}

\section{Introduction}

Patients who come to the dental office nowadays want more than just a healthy and functional restorative solution. The aesthetic role is playing an increasingly important paper in the choice of dental restorations. As the all-ceramic restorations are biocompatible and aesthetic, this alternative to metal-ceramic restorations is rapidly growing in popularity as the methods of manufacture these last ones, especially the CAD-CAM solutions.

\section{Case Report}

1 patient comes to the department of restorative and aesthetic of International Universidad Internacional de Cataluña after receiving endodontic treatment of the 3.6 in the department of endodontics of the same university. Presents an ocluso - bucco distal cavity. Due to the thickness of the remaining walls decided to perform a indirect onlay restoration through CAD-CAM technology. First it was made a direct composite reconstruction after endodontics. Next, the present composite reconstruction and tooth were prepared and scanned with Lyra 3Shape ${ }^{\circledR}$ system. The restoration was fully designed with Dental Designer ${ }^{\circledR}$ of the same brand. Then the onlay was milled using the milling machine Lyra and we makeup it with stains of the VITA brand after being sintered in the oven. Finally, the restoration was completed with a glaze and cemented with a dualcuring cement system. After removal of excess cement, occlusion was checked. The choice of the material for the restoration was decided according to the aesthetic and functional needs of the patient.

\section{Conclusions}

Bonded non-metallic restorations present the advantages of maintaining tooth tissue, reducing chair time and treatment costs, besides offering a more conservative view. 


\section{- Oral Presentation 126}

TITLE: Rehabilitation of posterior sectors with indirect restorations made with CAD/CAM systems

\section{AUTHORS: Sicilia Blanco E.}

\section{Introduction}

Traditionaly, it was expected from indirect restorations to have a better longevity than direct restorations, and from direct restorations to be less invasive than the indirect ones. However, nowadays with the improvements in mini-mally invasive dentistry and in the adhesive systems, the indications for ones or the others have changed.

\section{Case Report}

To clarify in which cases to use one tecnique or the other, as well as the protocols that should be followed in each case, we present a clinical case of a male patient, 45 yeard old, that after completing an orthodontic treatment, comes to the Clinic of the Universitat Internacional de Catalunya to rehabilitate his posterior sectors, that present several filtered old restorations.

After studying the case and removing the old restorations, we decide which technique to use in each case. We highlight the case of the second quadrant in which the teeth 2.4, 2.5, 2.6 and 2.7 are indications for 4 indirect restorations, that we design and elaborate with a CAD/ CAM system. We also highlight and document some basic concepts, as the immediate dentin sealing, before the adhesive technique, and elevation of deep subgingival margins. The preparation of the restorations and the cementation process are also documented.

\section{Conclusions}

Contemporary dentistry does not justify anymore the use of indirect restoratons based exclusively on the size of the defect or the longevity of the restorations. On the contrary, other factors as the predictability of extense restorations should be taken into account. In those cases, $\mathrm{CAD} / \mathrm{CAM}$ systems have prooved to give excellent results and are increasingly becoming more popular.

\section{- Oral Presentation 127 TITLE: CAD CAM flow today}

\section{AUTHORS: Ariño Domingo B, Estefania Murillas $S$, Fenoy Illacer P, Jane Noblom L.}

\section{Introduction}

The CAD CAM technology is already a reality that is coming to our clinics with the aim of improving the quality of our treatments, adjustment, accuracy, speed and simplicity.
CAD CAM systems offer its advantages the possibility of working with materials being milled monoblock increase their resistance as with lithium disilicate.

The world of CAD CAM materials is continually evolving. Following is a case of making a crown developing step by step workflow CAD CAM.

\section{Case Report}

Patient comes to the clinic of the International University of Catalonia because of metal porcelain crown in the ill-fitting and right upper canine cheapping.

It carried out the assessment of pillar and after checking their good condition CAD CAM crown is planned in Emax- CAD.

Step by step description of the preparation of the stump, making digital prints, crown design, manufacture thereof, makeup, glazed and cementation.

a temporary crown was planned with the aim of maturation and stabilization of the soft tissue and subsequently replaced with a definitive. a brief description of the various materials CAD CAM for provisional and definitive we can find in the market is done and what advantages we offer.

\section{Conclusions}

How has influenced the CAD CAM in the day. The adjustment and precision provided by new technologies. What materials are available and advantages over conventional materials provide us. 


\section{MULTIDISCIPLINARY TREATMENT}

\section{- Oral Presentation 128 \\ TITLE: Aesthetic in anterior teeth: Multidisci- plinary treatment}

\author{
AUTHORS: Agulló C, Marques H, Sena O, Valles \\ M, Ramírez A.
}

\section{Introduction}

Wear on the anterior teeth directly affects the aesthetics and their function. Patients ending orthodontic treatment and do not see fulfilled their aesthetic expectations for wear, they ask quick solutions and that they are minimally invasive. The treatment of choice are direct composites, for their aesthetics results and it does not compromise future treatments.

\section{Case Report}

A 28 years old Caucasian male, with a class III edge to edge bite. After his orthodontic treatment a Class I is achieved: molar and canine. He has a correct Overjet but a deficient Overbite due to wear teeth. The previus anatomy is returned with composites.(Enamel Plus HRi from Micerium $\left.{ }^{\circledR}\right)$.

\section{Conclusions}

The form and function are closely related. What orthodontics can not corrected by previous wears, hipoplasias or malformations could be by aesthetic dentistry with composites. It can be done at the end or during the treatment.

\section{- Oral Presentation 129}

TITLE: Intraorthodontic Mock-up: a new application for a more conservative treatments

\author{
AUTHORS: Arapé Páez J, Faus Matoses V, Faus \\ Matoses I, Faus Llacer V.
}

\section{Introduction}

It's commonly known that adhesion to enamel provides a successful long-term restorations, much greater than when is bonded to dentin. For this interdisciplinary ortho-restorations treatments may be the key to position ideally the teeth, thinking about the preparation and creating space for the restorative material, reducing the necessity of trim healthy dental tissue.

So far, the mock-up had several applications: guide the restorative treatment plan, serve as a guide for minimum tooth preparation and acceptance the future restorations.

A new application is described, to help the orthodontist to finish his part of the treatment, positioning the teeth in the ideal position for the restorer, in that way the preparation will be as conservative as possible.

\section{Case Report}

46 years old male 46 , with no medical history. The main concern in consultation was aesthetic and functional solution, pointing out that food is stuck up between the teeth. After obtaining clinical, radiographic and photographic registers, we propose aesthetic and functional objectives, thus, to make a personalized treatment plan that included, in addition to restorative treatment, an orthodontic phase that achieved an improved dental position guided by the placement of a mock-up in the last stage. This mock-up helped the orthodontist to visualize the ideal tooth position depending of the final restorations.

\section{Conclusions}

The use of a intra-orthodontic mock-up, is a novel technique to consider in interdisciplinary orthodonticrestorative treatments, giving the orthodontist the information of where to put teeth in the ideal position, letting the restorer place the final restorations without trimming or with minimal trimming.

\section{- Oral Presentation 130 \\ TITLE: Gummy Smile correction. An interdis- ciplinary conservative approach}

\section{AUTHORS: Rodrigues M, Faus V, Faus Matoses V, Faus I.}

\section{Introduction}

Esthetic conservative restoration is more in demand than ever with patients and has become a challenge for clinicians to resolve complicated cases. Interdisciplined planning is essential to obtain a result that satisfies the functional and esthetic expectations of the patient with minimum intervention.

\section{Case Report}

A 56 year old woman came to clinic to improve her smile. After clinical exploration a Gummy Smile was observed with Vestibulization of anterior teeth and the presence of diastemas, occlusion of Angle class III with cross bilateral bite and dental absences. Through a radiograph horizontal bone loss was observed due to periodontitis with mobility degree III in tooth 1.6. The aims of the treatment were to obtain an appropriate occlusion in class I and diminish the gummy smile in order to obtain optimum esthetic results. The treatment consisted of creating a new vertical dimension with direct posterior restorations. Afterwards, orthodontic treatment began in order to obtain an appropriate oclusal fit, placing microscrews in the maxilla as bone anchorage allowing us the intrusion of anterior upper teeth in order to decrease the amount of visible gum and obtaining a 
gingival insertion. In the final stage of the orthodontic treatment, implants were placed and remodelling of soft tissues was carried out. After orthodontic treatment, a diagnostic wax and mock-up were elaborated, that allowed us to value the final esthetic of the patient as a guide for preparation of minimum intervention in the anterior sector. Once the teeth were prepared, impressions were taken for the confection of adhesive feldspathic ceramic restorations.

\section{Conclusions}

Interdisciplinary planning is essential to achieve esthetic results with a conservative preparation. The approach with different professional standards allows us to offer solutions for complex cases with minimal structural tooth loss.

\section{- Oral Presentation 131 \\ TITLE: Interdisciplinary and conservative treatment in a case of class III malocclusion}

\author{
AUTHORS: Ramírez Lagos B, Faus V, Faus Matoses \\ V, Faus I.
}

\section{Introduction}

Communication and knowledge within the dental team of conservative procedures will help us to carry out complex treatments which at the same time will not be damaging to a healthy tooth structure, seeking to integrate biological, aesthetic and functional principles, thus achieving optimal, conservative and long term results.

\section{Case Report}

Male 48 years old, with no medical history of interest came to the clinic with the aim of being able to bite well and to change the aesthetics in the anterior teeth without undergoing surgical procedures. Clinical investigations and radiographic scans were carried out and were obtained and studied. It was observed that the patient had functional class III with anterior cross bite and deterioration in the incisal edges of the lower anterior teeth, compatible with that class III and deterioration on the occlusal of the posterior teeth.

We began an interdisciplinary treatment the started with the orthodontic phase with the goal of bringing the mandible to central position and increasing the vertical dimension and mesialization of the maxilla, thus obtaining suitable spaces for direct restoration of the lower posterior teeth and decreasing the preparation of the upper front teeth. Before finishing with the orthodontic treatment, direct composite restorations (Ceram-X Universal) of posterior teeth were made which allowed us to maintain the vertical dimension previously set and to finish the orthodontic treatment and to to achieve a stable occlusion. After orthodontic treatment of anterior teeth with direct composite and the upper anterior teeth restored with feldspathic restorations.

\section{Conclusions}

The management of a patient in an interdisciplinary way is the best way to reach a diagnosis and plan appropriate treatment, preserving as much dental tissue which will be reflected in a favorable outcome that meets the conservative needs of both the patient and clinicians.

\section{- Oral Presentation 132}

TITLE: An interdisciplinary approach for rehabilitating an adult patient with a moderate malocclusion: a case report

AUTHORS: Menéndez López-Mateos ML, González Villafranca P, Menéndez López-Mateos C, González López S, Menéndez Núñez M.

\section{Introduction}

Adult patients often come to the general practitioner looking for a complete dental treatment, which sometimes involves a multidisciplinary treatment shared with other professionals. We report a case of an adult patient with a multidisciplinary approach that consisted of orthodontic treatment, gingivectomy and aesthetic rehabilitation treatment.

\section{Case Report}

Adult patient with multiple teeth restorations and gingival smile. In orthodontic examination, the patient had Class II malocclusion 2nd division. Molar and canine Class II relationship was observed. And linguoversion of the upper and lower incisors. The extrusion of upper incisors produced, greater exposure of gingiva smile. Excess of incisal overbite prolonged in time has caused incisal wear. With orthodontic treatment the torque of the upper and lower incisors was improved. Light intrusion on upper incisors. And improve molar and canine relationship. Later, gingivectomy was performed to get an aesthetic proportion of the teeth and gingival harmony. Finally, E-max crowns from 1.4 to 2.4 were cemented with adhesive techniques. A maintenance plan included, superior thermoformed splint and inferior lingual fixed retention from 3.3 to 3.4 . In the final result patient's smile has improved the incisal overbite and molar and canine relationship on the left and right side has partially ameliorated.

\section{Conclusions}

The treatment of complex cases in adult patients requires a multidisciplinary approach. For this reason, it is needed the coordinate participation of several specialists in order to get a good aesthetic and functional results. 


\section{- Oral Presentation 133}

TITLE: Multidisciplinary treatment orthodontic-endodontic-aesthetic: a case report

AUTHORS: Fernández Pino N, Martín Jiménez M, Gallardo Rodríguez M, Segura-Egea JJ.

\section{Introduction}

The interplay of different disciplines in dentistry is essential to get a good functioan and aesthetic outcome of our treatments. Then,a case in wich disciplines such as Orthodontics,Endodontcs and dental aesthetic combine to improve aesthetically and functionallythe smiel is ppresented.

\section{Case Report}

A 55 years-old female patient came to the dental office because she wished to improve the aesthetic of her smile.A clinical examination observed a slight malocclusion,decoloration of the part 11 due to pulp necrosis and mild bonde-dental discrepancy cpursing with interincisal diastema. As treatment fix orthodontics, root canal treatment of the part 11 followed by two sessions of internal bleaching,external bleaching and veneers composite in 12 to 22 are proposed.Firstly,root canal treatmentof the 11 is performed,with the continuous rotatory MTWO

system calibrated with 35/05 file.Next,two external bleaching treatment was performed with self-ligated bracket (Damon system) during 18 months, followed by home external withening treatment. Finally, Veneers composite in 12 to 22 were performed,with Micerium composites. The aesthetic and functional outcome was excellent and patient expectations were achieved.

\section{Conclusions}

teh current trendof dentistry is to work within multidisciplinary temas,whereeach especialist carries out if treatments in the most optimal way and the aesthetic and functional result is excellent.

\section{OTHERS}

\section{- Oral Presentation 134 \\ TITLE: Invisibility of a class IV: report of a case}

\author{
AUTHORS: Garza Garza LC, Forero Guevara M, \\ Jané Noblom L.
}

\section{Introduction}

The clinical and aesthetic success of the composites restorations is conditioned by two fundamental requirements: knowledge of the optical properties of natural teeth and the knowledge, by the professional, of the system adopted. The aim of this oral presentation is to present, step by step, a direct reconstruction of the upper central incisor fractured using a direct composite system. For all of this, bringing emphasis not only on the concept of macro and micro anatomy, but also on the finishing and polishing, in order to achieve naturalness in the restorations.

\section{Case Report}

A clinical case of a Class IV restoration on tooth 11, carried out with the IPS Empress Direct System (Ivoclar Vivadent). This system is a photopolymerizable compound, a very aesthetic nano-hybrid composition suitable for direct restorations in the anterior and posterior sector. It is based on 32 tones and 5 levels of transparency, with this new concept it is not a requisite to use a specific layer technique, has suitable tolerance to light and is highly radiopaque. The system is simple to use and allows to achieve predictable results. The easy method consists of choosing the the hue of dentin composite that is closest to the final outcome of desire and to use the enamel at the same way. This thin layer of enamel on dentin gives more depth and "life" to repair.

\section{Conclusions}

When the clinician dominates these two factors, he/she is able to recognize which composite should be used and also is capable to select the amount of material desired to imitate the characteristics of the tooth to be restored. The challenge of a restoration lies not only finding the correct color, which also involves a balance between anatomy to produce a highly esthetic restoration.

\section{- Oral Presentation 135}

TITLE: Maryland bridge using a natural tooth. A case report

\section{AUTHORS: Cendejas I, Lucena C, Robles V, Pulgar R.}

\section{Introduction}

Anterior tooth loss, either by traumatic or periodontal causes, constitutes an aesthetic emergency. Toe situa- 
tion requires an immediate intervention while the patient waits for an ultimate dental prosthetic solution. In this context, adhesive bridges type Maryland, in all its variants and designs, constitute a reasonable altemative. Its retention is based on adhesive principles and it responds, certainly, to the philosophy ofMinimally Invasive Dentistry.

\section{Case Report}

Female aged 50 comes to our clinic, presenting great mobility in tooth 31 and extrusion. The patient, who was diagnosed and treated of disease periodontal, referred to have accidentally hit her tooth a few days ago. After clinical exploration, we verified that there was no altemative but extraction. The patient told us that she had a social commitment two days later, for that reason we considered the possibility of realizing a provisional Maryland bridge using her own tooth, option that the patient accepted.

After carefully extracting the piece, a section ofthe root up to $5 \mathrm{~mm}$ from the cement enamel limit was made. Later, the pulp tissue was removed from the tooth crown, the pulp chamber cleaned and then sealed using adhesive technique and flow composite. The root end point was shaped as ovoid pontic using microfilled composite to obtain a highly polished surface. Once the tooth was prepared, we placed the Maryland bridge using fiberglass, adhesive and fluid composite protocol.

\section{Conclusions}

The Maryland bridge using glass fiber can be a provisional restoration of long duration and very conservative in its procedure that satisfies the aesthetic expectations ofthe patient and allows to solve simple form situations ofhigh aesthetic compromise, as long as it is realized following correct indications.

\section{- Oral Presentation 136 \\ TITLE: Autotransplantation: a conservative approach to maintain natural dentition}

\author{
AUTHORS: Aguilar A, Morales K, Duran Sindreu \\ F, Roig M.
}

\section{Introduction}

A great majority of dental traumatisms take place during the age of 8 and 12 on the anteriosuperior sector, providing a dental loss, thereby affecting the potential bone and gingival growth, such as gingival aesthetic problems discrepancies and absence of interdental papillae.

Autotransplantation, is defined as the "transfer of a tooth from it's socket to another location within the same individual, either to an extraction site or to a surgical socket" ; this technique generates great advantages, it allows to keep the periodontal support tissue and the surrounding bone, preserving the potential of transplanted tooth eruption, and also increasing the potential for better aesthetics.

The first case was reported in 1950 , and since then the success rate has gradually increased by advances in diagnostic and surgical techniques.

\section{Case Report}

A 10 years old female patient case is presented with an autotransplantation performed in the 2.1. She presented a history of dental trauma avulsion and subsequent replantation of 2.1 with ankylosis in the same.

It was decided to perform a non traumatic extraction of 2.1 and 2.5. the 2.5 was placed in the recipient socket in infraocclusion and fixed by suturing with non absorbable monofilament 5/0 Subsequently, clinical and radiographic follow-ups were performed, at 10 months the restorative treatment was initiated, which consisted on preparing the 2.5 and it's posterior restoration with composite, this way we obtained great aesthetics results, with the proper anatomy of an upper central incisor. Then, the orthodontic treatment was initiated.

\section{Conclusions}

The use of autotransplantation provided the best option to replace a lost tooth, maintaining the potential of erupting tooth as well as bone and gingival growth resulting in favorable biological, functional and aesthetic results.

\section{- Oral Presentation 137 \\ TITLE: Restoration of the anterior sector using the injection technique}

\section{AUTHORS: Tudela-Muñoz FJ, Benavides-Reyes C, González-Rodríguez MP.}

\section{Introduction}

Adult patients usually come to the dentist demanding aesthetic treatment. Sometimes they can not afford the cost of an aesthetic prosthetic treatment. We present a case solved with adhesive restoration techniques, respecting the remaining dental structure and restoring the mechanical, functional and aesthetic properties.

\section{Case Report}

39 year old woman came, to the School of Dentistry at the University of Granada, unhappy with her smile asking for an economic esthetic treatment. Clinical examination shows composite veneers in upper incisors and color change in the upper left lateral incisor subsequent to endodontic treatment. Radiographic examination showed no pathology.

After photographic study of facial, oral and dental esthetic; Digital Smile Design was performed with Photoshop CC2015 and a Mock-up was prepared with this information. This was probed in the patient and the 
modifications were made on the wax model. With the final wax, a silicone impression was made, which served as a guide for preparing the teeth; and another impression was made with transparent silicone in a thermoformed splint. Holes were made in the incisal edge area of the transparent impression.

The clinical procedure involved the removal of old composite veneers, conditioning of tooth surfaces by etching and adhesive and composite reconstruction, using Empress Direct, based on the Enrique Guzman technique. The composite was heated in a water bath at $50^{\circ} \mathrm{C}$ before the application and applied through the holes in the splint. It was polymerized with LED light. Finally the occlusion was checked and the restorations were polished.

Conclusions

The technique presented is aesthetic, cheap and easily reproducible. 